\title{
CTF Void Drift Validation Study
}

Robert Salko, Oak Ridge National Laboratory Marcus Gergar, The Pennsylvania State University Chris Gosdin, The Pennsylvania State University Maria Avramova, North Carolina State University

$10 / 26 / 2015$

Approved for public release.

Distribution is unlimited.
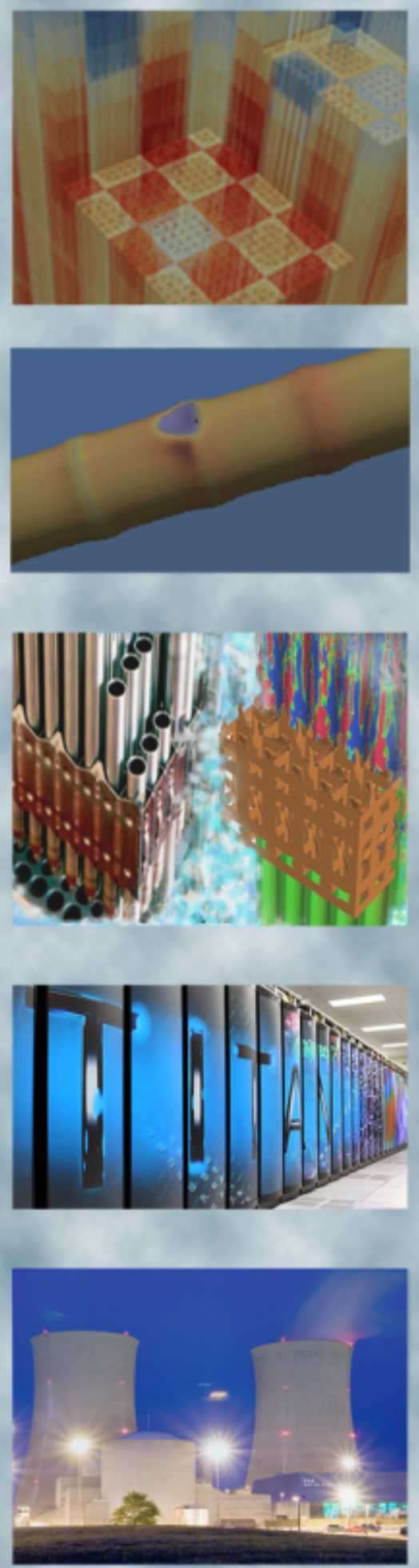
REVISION LOG

\begin{tabular}{|c|c|c|l|}
\hline Revision & Date & Affected Pages & \multicolumn{1}{|c|}{ Revision Description } \\
\hline 0 & $9 / 30 / 15$ & All & Initial Release \\
\hline 1 & $10 / 05 / 15$ & All & Adding mixing validation and more GE $3 \times 3$ tests \\
\hline 2 & $10 / 26 / 15$ & All & Addressing reviewer comments \\
\hline & & & \\
\hline
\end{tabular}

\section{Document pages that are:}

Export Controlled

IP/Proprietary/NDA Controlled

Sensitive Controlled

\section{Requested Distribution:}

To: Richard Lahey, Kevin Clarno, Scott Palmtag

Copy: Jeff Banta 


\section{EXECUTIVE SUMMARY}

This milestone report is a summary of work performed in support of expansion of the validation and verification $(\mathrm{V} \& \mathrm{~V})$ matrix for the thermal-hydraulic subchannel code, $\mathrm{CTF}$. The focus of this study is on validating the void drift modeling capabilities of $\mathrm{CTF}$ and verifying the supporting models that impact the void drift phenomenon. CTF uses a simple turbulent-diffusion approximation to model lateral cross-flow due to turbulent mixing and void drift. The void drift component of the model is based on the Lahey and Moody model. The models are a function of two-phase mass, momentum, and energy distribution in the system; therefore, it is necessary to correctly model the flow distribution in rod bundle geometry as a first step to correctly calculating the void distribution due to void drift. Considering this, a stepwise approach is taken to validate the void drift model in CTF by ensuring

1. the single-phase flow distribution is correctly predicted compared with an analytical solution,

2. the single-phase mixing is correctly predicted compared with an analytical solution,

3. the calculated flow distribution compares well with experimental single-phase data, and

4. the calculated void distribution compares favorably with experimental data.

The existing CTF Validation Manual already contains void-drift validation data using the General Electric (GE) $3 \times 3$ facility to address Item 4 in the above list. This study addresses Items $1-3$ and expands on Item 4 using new data. The first attempt at this expansion involved modeling the $2 \times 2$ air/water facility operated at Rensselaer Polytechnic Institute (RPI). However, it is discovered that CTF has a great deal of difficulty in converging this case likely due to the large geometry, low pressure, and two-phase injection. To better address Item 4, the Boiling Water Reactor Full-Size Fine-Mesh Bundle Test (BFBT) void distribution cases are run with CTF, which are steam/water two-phase tests run at prototypical BWR operating conditions in an $8 \times 8$ rod-bundle facility. Additionally, the GE $3 \times 3$ validation study is expanded with new test cases as well as additional analysis. 
This page intentionally left blank 


\section{CONTENTS}

EXECUTIVE SUMMARY iii

CONTENTS $\quad \mathrm{v}$

FIGURES

TABLES $\quad$ ix

ACRONYMS $\quad x$

1 INTRODUCTION 1

2 SINGLE-PHASE FLOW DISTRIBUTION TESTING 2

2.1 Two-Channel Friction Model Verification . . . . . . . . . . . . . . . . . . . 3

2.2 Turbulent Mixing Verification . . . . . . . . . . . . . . . . . . . 6

$2.32 \times 3$ Validation . . . . . . . . . . . . . . . . . . . . . . . . . . . 11

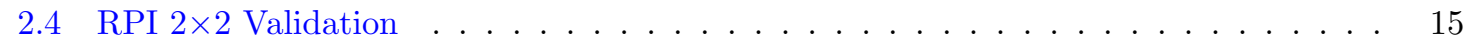

2.5 GE $3 \times 3$ Validation . . . . . . . . . . . . . . . . . . . . . . . . . . 24

3 VOID DRIFT VALIDATION 31

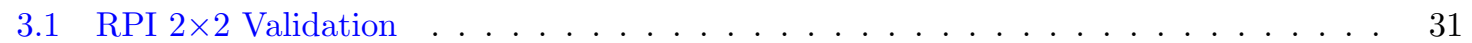

3.2 GE $3 \times 3$ Validation . . . . . . . . . . . . . . . . . . . . . . . . . . . . 38

3.3 BWR Full-Size Fine-Mesh Bundle Test (BFBT) $8 \times 8$ Validation . . . . . . . . . . . . 46

4 CONCLUSION $\quad 80$ 


\section{FIGURES}

Diagram of the two-channel flow split problem. . . . . . . . . . . . . . 5

Two-channel flow-split results . . . . . . . . . . . . . . . . 6

Model of problem for testing single-phase turbulent mixing of enthalpy. . . . . . . 7

Turbulent-mixing problem results . . . . . . . . . . . . . . . . . . 10

Mixing problem mass flow rates . . . . . . . . . . . . . . . . . . . . 10

Mixing problem mass flow rates with no mixing . . . . . . . . . . . . . . . 11

$2 \times 3$ facility cross section . . . . . . . . . . . . . . . . . . . . . 12

$2 \times 3$ facility side view . . . . . . . . . . . . . . . . . . . . . 12

$2 \times 3$ opearting conditions . . . . . . . . . . . . . . . . . 14

$2 \times 3$ results using Rogers and Rosehart . . . . . . . . . . . . . . . . . . . 14

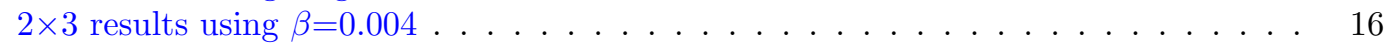

$2 \times 3$ results using $\beta=0.007 \ldots \ldots \ldots \ldots \ldots \ldots \ldots \ldots$

$2 \times 3$ results using CTF friction model _ . . . . . . . . . . . . . . . . 17

Watts Bar simulation sensitivity to $\beta \ldots \ldots \ldots \ldots \ldots \ldots$. . . . . . . . . . . . .

$2 \times 2$ Case 1 results with no mixing . . . . . . . . . . . . . . . 20

$2 \times 2$ Case 2 results with no mixing . . . . . . . . . . . . . . 20

$2 \times 2$ Case 1 results with mixing . . . . . . . . . . . . . . . . . 21

$2 \times 2$ Case 2 results with mixing . . . . . . . . . . . . . . . 21

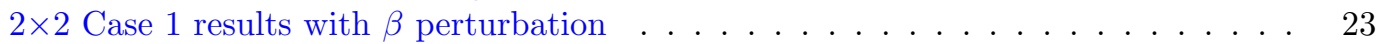

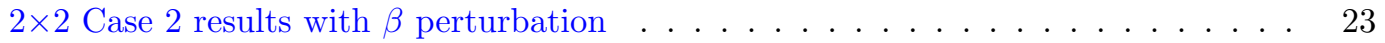

GE $3 \times 3$ Case 1 B results with no mixing . . . . . . . . . . . . 25

GE $3 \times 3$ Case $1 \mathrm{C}$ results with no mixing . . . . . . . . . . . . 25

GE $3 \times 3$ Case 1 D results with no mixing . . . . . . . . . . . . . 26

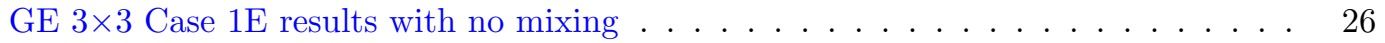

GE $3 \times 3$ Case 1 B results with mixing . . . . . . . . . . . . . 27

GE $3 \times 3$ Case 1 C results with mixing . . . . . . . . . . . . . . 27

GE $3 \times 3$ Case $1 \mathrm{D}$ results with mixing . . . . . . . . . . . . . . . 28

GE $3 \times 3$ Case 1 E results with mixing . . . . . . . . . . . . . . 28

GE $3 \times 3$ single-phase test summary . . . . . . . . . . . . . . . . 30

GE $3 \times 3$ single-phase test summary using Rogers and Rosehart . . . . . . . . . . . . 30

CTF convergence terms for Test 3 of the $2 \times 2$ facility when run at atmospheric pressure. 32

Exit void in Test 3 of $2 \times 2$ facility throughout simulation. . . . . . . . . . . . . 32

Exit void in Test 3 of $2 \times 2$ facility with gaps disabled. . . . . . . . . . . . . 33

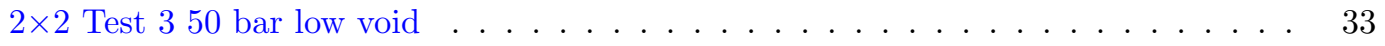

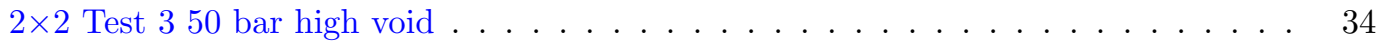

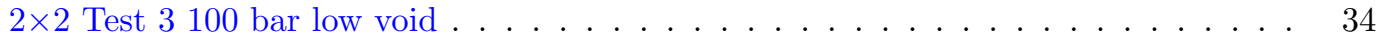

$2 \times 2$ Test 3100 bar medium void $\ldots \ldots \ldots \ldots \ldots \ldots \ldots$

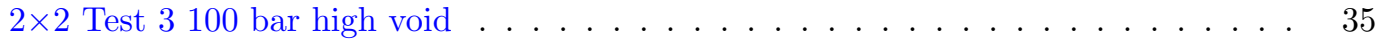

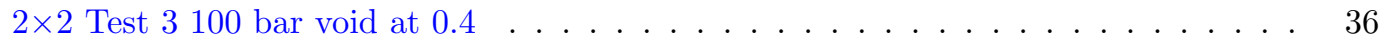

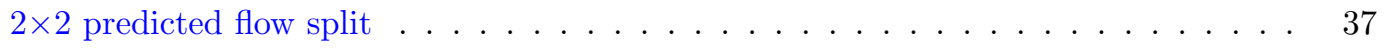

$2 \times 2$ predicted flow split in extended model _ . . . . . . . . . . . . . 37

$2 \times 2$ Test 3 void for extended facility . . . . . . . . . . . . . . . . 38

GE $3 \times 3$ quality summary . . . . . . . . . . . . . . . . . . . 40

GE $3 \times 3$ quality summary without void drift . . . . . . . . . . . . . 40

GE $3 \times 3$ mass flux summary . . . . . . . . . . . . . . . . . . . . 41

GE $3 \times 3$ mass flux summary without void drift . . . . . . . . . . . . . . 41

GE $3 \times 3$ mass flux summary using Rogers and Rosehart . . . . . . . . . . . . . . 42

GE $3 \times 3$ quality summary using Rogers and Rosehart . . . . . . . . . . . . . . . 42

GE $3 \times 3$ Case $2 \mathrm{C} 1$ flow split . . . . . . . . . . . . . . . . . . . 44

GE $3 \times 3$ Case 2 C2 flow split . . . . . . . . . . . . . . . . . . 44

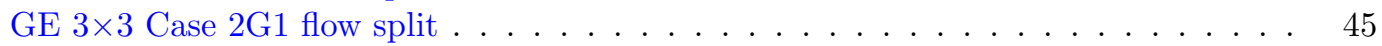

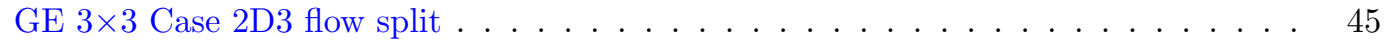


53 Assembly $0-1,0-2$, and $0-3$ description . . . . . . . . . . . . . . . . . . . . 47

54 Assembly 0-1, 0-2, and 0-3 description (continued) . . . . . . . . . . . . . . 48

55 Assembly 1, 2, and 3 description . . . . . . . . . . . . . . . . . 48

56 Assembly 1 power ............................. . . 49

57 Assembly 4 power ............................ . . . 50

58 Assembly 4 description . . . . . . . . . . . . . . . . . . . . . . . 50

59 BFBT void measurements . . . . . . . . . . . . . . . . . . . . . 51

60 Assembly 0-1, 0-2, 0-3, and 1 flow areas ................... . . 51

61 Assembly 4 flow areas . . . . . . . . . . . . . . . . . . . . . 52

$62 \mathrm{C} 2 \mathrm{~A}$ ferrule grid geometry . . . . . . . . . . . . . . . . . . . . 53

63 BFBT loss coefficients . . . . . . . . . . . . . . . . . . . . . 54

64 Comparison of measured and predicted bundle-averaged outlet void. . . . . . . . . . 55

65 Comparison of measured and predicted bundle-averaged outlet thermal equilibrium quality. . . . . . . . . . . . . . . . . . . . . . 5 55

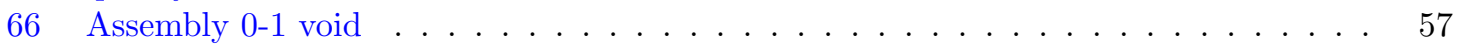

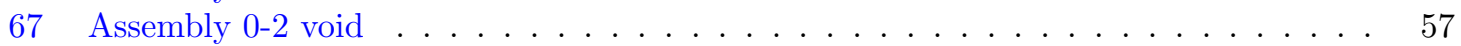

68 Assembly $0-3$ void . . . . . . . . . . . . . . . . . . . . . . . . . 58

69 Assembly 1 void ............................. . . . 58

70 Assembly 4 void ............................ . . 59

71 BFBT void summary ........................... . . . 60

72 BFBT void summary with droplets . . . . . . . . . . . . . . . . . . . 60

73 BFBT corner channel void . . . . . . . . . . . . . . . . . . . . 61

74 BFBT corner channel void with droplets . . . . . . . . . . . . . . . . . . . . 61

75 BFBT side channel void . . . . . . . . . . . . . . . . . . . . . . 62

76 BFBT side channel void with droplets . . . . . . . . . . . . . . . . . . . . 62

77 BFBT inner channel void . . . . . . . . . . . . . . . . . . . 63

78 BFBT inner channel void with droplets . . . . . . . . . . . . . . . . 63

79 BFBT unheated channel void . . . . . . . . . . . . . . . . . . . . . 64

80 BFBT unheated channel void with droplets . . . . . . . . . . . . . . . . . 64

81 BFBT void with no void drift . . . . . . . . . . . . . . . . . . . . 65

82 BFBT corner void with no void drift . . . . . . . . . . . . . . . . . . 65

83 BFBT side void with no void drift . . . . . . . . . . . . . . . . . . . 66

84 BFBT inner void with no void drift . . . . . . . . . . . . . . . . . . . . 66

85 BFBT unheated void with no void drift . . . . . . . . . . . . . . . . . 67

86 Example of diagonal lines from where subchannel void data is extracted. . . . . . . 69

87 Measured and predicted void along diagonals of Test 0011-55. . . . . . . . . . . . . 70

88 Measured and predicted void along diagonals of Test 0011-55 with void drift disabled. 70

89 Measured and predicted void along diagonals of Test 0011-61. . . . . . . . . . . . . 71

90 Measured and predicted void along diagonals of Test 0011-61 with void drift disabled. 71

91 Measured and predicted void along diagonals of Test 0021-16. . . . . . . . . . . . . . 72

92 Measured and predicted void along diagonals of Test 0021-16 with void drift disabled. 72

93 Measured and predicted void along diagonals of Test 0021-21. . . . . . . . . . . . . . 73

94 Measured and predicted void along diagonals of Test 0021-21 with void drift disabled. 73

95 Measured and predicted void along diagonals of Test 0031-16. . . . . . . . . . . . . . 74

96 Measured and predicted void along diagonals of Test 0031-16 with void drift disabled. 74

97 Measured and predicted void along diagonals of Test 0031-21. . . . . . . . . . . . . 75

98 Measured and predicted void along diagonals of Test 0031-21 with void drift disabled. 75

99 Measured and predicted void along diagonals of Test 1071-55. . . . . . . . . . . . . 76

100 Measured and predicted void along diagonals of Test 1071-55 with void drift disabled. 76

101 Measured and predicted void along diagonals of Test 1071-61. . . . . . . . . . . . . . 77

102 Measured and predicted void along diagonals of Test 1071-61 with void drift disabled. 77

103 Measured and predicted void along diagonals of Test 4101-53. . . . . . . . . . . . . 78

104 Measured and predicted void along diagonals of Test 4101-53 with void drift disabled. 78

105 Measured and predicted void along diagonals of Test 4101-61. . . . . . . . . . . . . . 79 
106 Measured and predicted void along diagonals of Test 4101-61 with void drift disabled. 79 


\section{TABLES}

$1 \quad$ NUREG Subchannel Flow Dimensions . . . . . . . . . . . . . . . . . . . . . . 18

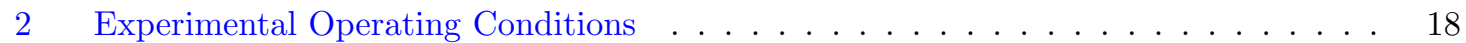

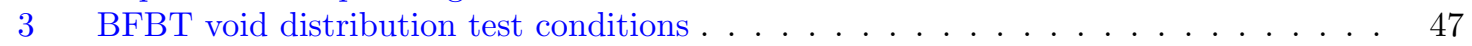

4 BFBT Assembly Type 1 axial power distribution. . . . . . . . . . . . . . . . . . 49

5 Summary of the CTF predictions of BFBT void distribution cases $\ldots \ldots \ldots$. . . . 67 


\section{ACRONYMS}

BFBT BWR Full-Size Fine-Mesh Bundle Test

BWR boiling water reactor

GE General Electric

LWR light water reactor

PWR pressurized water reactor

RPI Rensselaer Polytechnic Institute

RMSE root-mean-square of error

rRMSE relative root-mean-square of error

$\mathbf{V} \& \mathbf{V}$ validation and verification 


\section{INTRODUCTION}

This report documents the annual update to the CTF validation and verification (V\&V) test matrix. This update is focused on expanding the testing of the void drift model in CTF as well as the supporting models that most significantly affect the phenomena. CTF uses a simple diffusion model based on mixing-length theory for capturing turbulent mixing and void drift. This is summarized by Equation 1.

$$
W_{i j}^{D^{\prime}}=\epsilon \frac{s_{i j}}{z_{i j}^{T}}\left(\rho_{f}-\rho_{g}\right)\left[\alpha_{v, j}-\alpha_{v, i}-\left(\alpha_{v, j}-\alpha_{v, i}\right)_{\mathrm{equil}}\right]
$$

The suffixes, $i$ and $j$, are indices representing two adjacent subchannels. In the equation, $W_{i j}^{D^{\prime}}$ is the linear mixing rate (units of $\mathrm{kg} \mathrm{m}^{-1} \mathrm{~s}^{-1}$ ), $s_{i j}$ is the gap width (units of $\mathrm{m}$ ), $z_{i j}^{T}$ is the turbulent mixing length (units of $\mathrm{m}), \rho_{f}$ and $\rho_{g}$ are the two phase densities, and the bracketed terms, $\left(\alpha_{v, j}-\right.$ $\left.\alpha_{v, i}\right)$ and $\left(\alpha_{v, j}-\alpha_{v, i}\right)_{\text {equil }}$, are the turbulent mixing and void drift driving forces. The turbulent mixing driving force is simply the gradient in the parameter of interest between adjacent suchannelsthe turbulent mixing of momentum is driven by the gradient in momentum, $\rho v$; the turbulent mixing of energy is driven by the gradient in energy, $h$; and so on. The void drift driving term is defined by the model proposed by Lahey and Moody [1] as shown in Equation 2.

$$
\left(\alpha_{v, i}-\alpha_{v, j}\right)_{\mathrm{equil}}=K_{a}\left(\alpha_{v, i}+\alpha_{v, j}\right) \frac{G_{i}-G_{j}}{G_{i}+G_{j}}
$$

The term, $K_{a}$, is a geometry dependent scaling parameter that is typically taken as 1.4. The remaining terms are the void fractions, $\alpha$, and mass fluxes, $G$, of adjacent subchannels. A full derivation of the CTF model of turbulent mixing and void drift as it applies to the two-phase, three-field model is available in the CTF Theory Manual [2].

The intention of this study is to show that different components of these models are working as intended and comparing favorably to experimental data. The approach is to first show that these models compare to analytical solutions of simple problems (solution verification). This task, in and of itself, does not prove that the model is useful for real world applications; it only proves that it has been implemented in a bug-free fashion and gives the correct answer for that particular use case. The second step is to show that the model, under ideal conditions, gives favorable answers for realworld problems (i.e., experimental data). The first task is designed to be more of a separate-effects study, meaning a limited scope of the code is being exercised. The second task-comparing with experimental data - naturally becomes more of an integral-effects study.

Specific considerations made are

1. the single-phase flow distribution that is input into the turbulent mixing and void drift models is correct,

2. the single-phase turbulent mixing model works as intended, and

3. the void drift model improves two-phase modeling results by capturing the trend of void to migrate to larger-area, higher- velocity subchannels.

Simple problems with analytical solutions are formulated to demonstrate: 1) that the flow split is correctly calculated, and 2) that the turbulent mixing is correctly calculated. After this, CTF is used to model real rod-bundle experiments and is shown to calculate the flow split and turbulent mixing correctly. The scope of this study is limited to single-phase cases only. Performing two-phase turbulent mixing verification is difficult from a separate-effects perspective because it is impossible to separate the competing effects of turbulent mixing and void drift. Naturally, the void drift validation work is more of an integral effects study, combining the effects of single- and two-phase mixing and void drift. The best we can do in this case is to model unheated experimental facilities without spacer grids to at least reduce the number of physical phenomena being modeled.

This is attempted by modeling the low-pressure air/water $2 \times 2$ facility that was operated at Rensselaer Polytechnic Institute (RPI) [3]. This short rod bundle was unheated and did not contain 
any spacer grids; air was used as the gas-phase fluid to observe the void drift phenomena. However, as will be shown, considerable difficulty was encountered in modeling this facility using CTF. The code has great difficulty in converging on systems with two-phase injection and low pressure (atmospheric pressure), likely due, in part, to the large gradient between phase properties at these low pressures.

To conclude the study and still address the issue of validating the CTF void drift model, CTF is used to model tests with operating conditions that are representative of actual boiling water reactor (BWR) facilities: the General Electric (GE) $3 \times 3$ facility and the BWR Full-Size Fine-Mesh Bundle Test (BFBT) $8 \times 8$ facility. The GE facility already has been modeled and documented in the CTF Validation Manual [4]; however, the tests are expanded to include the single-phase cases and to show CTF's ability to capture the single-phase flow distribution correctly.

The $8 \times 8$ facility is unique from the $3 \times 3$ facility in several ways, including

1. a larger rod bundle geometry,

2. multiple guide tube and unheated rod configurations,

3. non-uniform axial and radial power distributions,

4. different spacer grid geometry,

5. different void measurement system, and

6. taller test facility.

These differences result in a valuable set of data being added to the testing matrix.

\section{SINGLE-PHASE FLOW DISTRIBUTION TESTING}

CTF models three drivers of lateral flow:

1. Pressure-driven directed cross-flow

2. Single- and two-phase turbulent mixing

3. Void drift

By modeling single-phase cases, we eliminate the two-phase turbulent mixing and void drift components. Single-phase mixing can be enabled or disabled from CTF input, which leaves only directed cross-flow. Directed cross-flow can result from any lateral pressure imbalance, which includes

1. unequal pressure drops in adjacent channels,

2. density gradients, and

3. advection of flow due to lateral boundary conditions.

If we design an unheated case with an injection mass flow rate boundary condition at the bottom of the model, lateral flow migration will be caused by unequal axial pressure drops. For a model with no form losses (spacer grids) and connected channels with different geometry, flow redistribution will be driven by unequal frictional losses, allowing for an analytical solution to be developed for the ideal flow split. This is done for both two- and multiple-channel models and is used to verify the CTF single-phase friction model.

Likewise, if the geometry of the channels is identical, the lateral flow migration will be caused only by single-phase turbulent mixing and only if there is a gradient in one of the solution variables between the subchannels. An analytical solution is built for this problem and compared with CTF results to verify the single-phase turbulent mixing model.

After verification testing is completed, CTF is used to model single-phase turbulent-mixing tests to demonstrate that the turbulent mixing model can predict real experimental results correctly.

Finally, two rod bundle experiments that measure the outlet mass flow rates of individual channels are modeled to demonstrate CTF's capability to predict real rod-bundle flow distributions. 


\subsection{Two-Channel Friction Model Verification}

Problem Description A model of two channels connected by a gap is created. One channel has a larger hydraulic diameter than the other. The inlet velocity is uniform in the two channels, the case is unheated, and the coolant is single-phase and highly subcooled. This creates a difference in Reynolds number at the inlet of the two channels, which creates different frictional pressure drops in the two channels, as the friction model is Reynolds dependent.

The different frictional pressure drops create a lateral pressure gradient that drives flow from the higher resistance channel to the lower resistance channel. Moving up the channels, velocity grows larger in the low-resistance channel, which increases frictional pressure drop in that channel. Simultaneously, velocity decreases in the high-resistance channel, which decreases frictional pressure drop. This continues until the frictional pressure drop is the same in both channels, at which point cross-flow ceases. At this point, the channels are said to be in mechanical equilibrium.

An analytical solution is derived for this point of mechanical equilibrium. We consider a control volume in each channel at this level where equilibrium has been reached. It is safe to neglect the lateral momentum equation because cross-flow has stopped. An axial momentum equation can be formed for each channel control volume. The general axial momentum equation is shown below.

$$
\rho\left[\frac{\partial \boldsymbol{V}}{\partial t}+u \frac{\partial \boldsymbol{V}}{\partial x}+v \frac{\partial \boldsymbol{V}}{\partial y}+w \frac{\partial \boldsymbol{V}}{\partial z}\right]=\rho g-\nabla p+\nabla \dot{\tau}_{i j}
$$

The density is removed from the left-hand side terms since it is assumed constant in the problem. The bracketed terms include: 1) time-change of momentum, 2) axial ( $x$ ) advection of momentum, 3 ) lateral ( $y$ ) advection of momentum, and 4) lateral $(z)$ advection of momentum. The three right-hand side terms are the relevant force terms, including: 1) gravity, 2) pressure, and 3) shear.

This equation can be significantly reduced considering

1. the case is steady-state, eliminating the temporal term;

2. there is no cross-flow, eliminating lateral convection terms; and

3. the axial velocity distribution in this control volume is constant, as density is constant and there is no cross-flow, meaning the axial momentum convection term can be eliminated.

This eliminates the entire left-hand side of the equation and leaves the following equation for an individual subchannel, where $x$ is taken as the axial direction:

$$
\frac{d P}{d x}=\rho g+\frac{d \tau_{w}}{d x}
$$

Because the two channels are in mechanical equilibrium, the pressure drops in the channels are equal, allowing us to equate the right-hand side of each individual channel equation. Note that the gravity head is identical in the two channels, allowing the term to be cancelled. Finally, integrating over the control volume height, $d x$, allows us to obtain the final relation between the two channels.

$$
\tau_{w, 1}=\tau_{w, 2}
$$

The wall drag, $\tau_{w}$, is determined from the CTF friction model, which is substituted into Equation 5 to produce the following expansion:

$$
\frac{f_{1} u_{1}^{2}}{2 D_{h, 1}}=\frac{f_{2} u_{2}^{2}}{2 D_{h, 2}}
$$

The terms, $f, u$, and $D_{h}$, represent the Darcy friction factor, liquid velocity, and channel hydraulic diameter, respectively. The subscripts indicate which channel the term represents. The CTF friction factor model is used in the problem to calculate $f$ as a function of Reynolds number. It has the following form:

$$
f=C_{1} R e^{C_{2}}
$$


$C_{1}$ and $C_{2}$ are model coefficients. Expanding the Reynolds number and substituting this into Equation 6 yields the following relationship between channel velocities:

$$
\frac{C_{1}\left(\frac{\rho u_{1} D_{h, 1}}{\mu}\right)^{C_{2}} u_{1}^{2}}{D_{h, 1}}=\frac{C_{1}\left(\frac{\rho u_{2} D_{h, 2}}{\mu}\right)^{C_{2}} u_{2}^{2}}{D_{h, 2}}
$$

Canceling terms and reducing leads to the following form:

$$
\left(\frac{u_{1}}{u_{2}}\right)^{2+C_{2}}=\left(\frac{D_{h, 2}}{D_{h, 1}}\right)^{C_{2}-1}
$$

The hydraulic diameters of the two channels are defined by the model geometry. For CTF, $C_{2}$ is -0.2. If we also consider the mass conservation equation, which tells us that the sum of the outlet mass flow rates is equal to the inlet mass flow rate, we can obtain the expected solutions for the absolute outlet mass flow rate of each channel. First, Equation 9 is set in terms of mass flow rate to produce the following:

$$
\left(\frac{\dot{m}_{1}}{\dot{m}_{2}} \frac{A_{2}}{A_{1}}\right)^{2+C_{2}}=\left(\frac{D_{h, 2}}{D_{h, 1}}\right)^{C_{2}-1}
$$

Next, the mass conservation equation is used to relate the mass flow rates in the individual channels to the total mass flow rate in the system:

$$
\begin{aligned}
& \dot{m}_{\mathrm{in}}=\dot{m}_{1}+\dot{m}_{2} \\
& \dot{m}_{\mathrm{in}}=\dot{m}_{2}\left(1+\left(\frac{D_{h, 2}}{D_{h, 1}}\right)^{\frac{C_{2}-1}{2+C_{2}}} \frac{A_{1}}{A_{2}}\right)
\end{aligned}
$$

CTF Model Description Channel 2 has a hydraulic diameter that is twice the size of the Channel 1 hydraulic diameter. The area and wetted perimeter of Channel 1 are set to values close to those expected of typical pressurized water reactor (PWR) rod-lattice geometry. The outlet pressure is $155 \mathrm{bar}$ and inlet mass flux is $3500 \mathrm{~kg} \mathrm{~m}^{-2} \mathrm{~s}^{-1}$. The inlet temperature is set to $200^{\circ} \mathrm{C}$ to keep the model sufficiently subcooled, and the case is unheated. Turbulent mixing and void drift is disabled so that pressure is the only driver for cross-flow. A diagram of this model is shown in Figure 1. The length of the model is set to $10 \mathrm{~m}$ to allow the flow to completely redistribute within the CTF solution space.

The axial mesh is set to different sizes, including $2.54 \mathrm{~cm}, 5.08 \mathrm{~cm}$, and $10.16 \mathrm{~cm}$; however, it is found that axial mesh size has no impact on the axial mass flow rate profiles. With the geometry defined, Equation 12 is used to calculate that the expected outlet mass flow rates in Channels 1 and 2 .

Discussion of Results The CTF solution includes the axial mass flow rate distribution in each channel. The analytical solution only gives us the expected flow rate distribution at the exit. Therefore, we cannot compare the CTF axial flow distribution to the analytical solution, but we can at least guarantee that CTF achieves the correct flow split when mechanical equilibrium is achieved. The mass flux in each channel is normalized before plotting using the following relationship:

$$
G_{i, \text { norm }}=\frac{G_{i}-\bar{G}}{\bar{G}} \times 100
$$

$\bar{G}$ is the average mass flux between channels, which is equal to the inlet mass flux. Therefore, the normalized mass flux in each channel is zero at the inlet and then re-distributes due to frictional resistance. Figure 2 shows the CTF-predicted flow distribution in the two-channel system. The correct analytical flow split is shown with the dashed lines.

These results demonstrate that CTF predicts the expected flow split between the two channels at about $7 \mathrm{~m}$ from the inlet. 


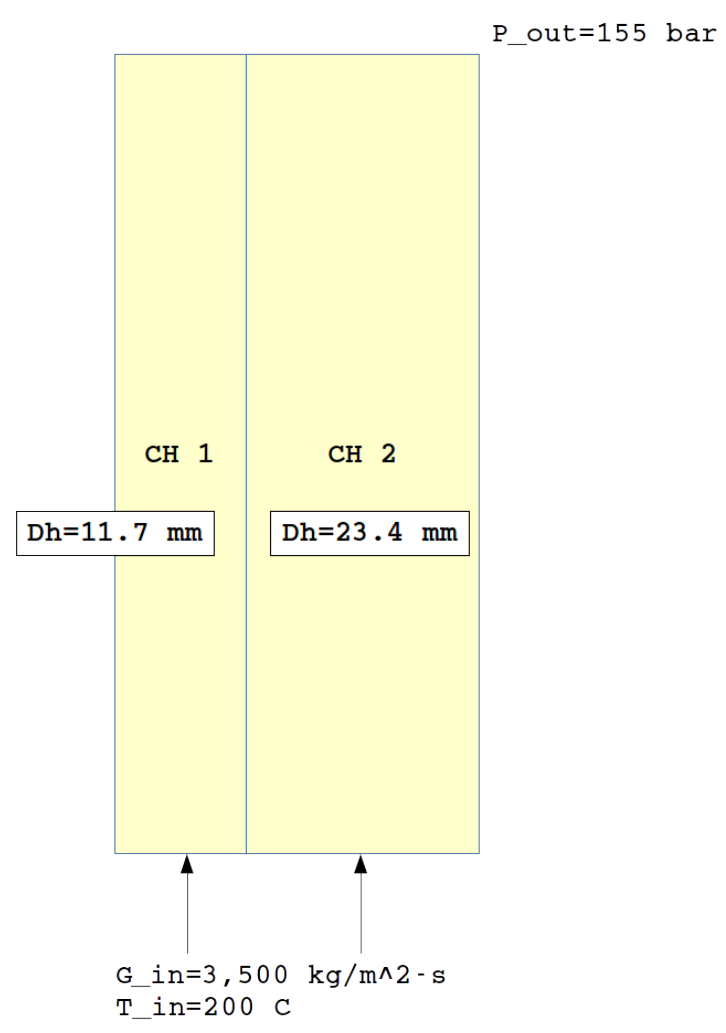

Figure 1. Diagram of the two-channel flow split problem. 


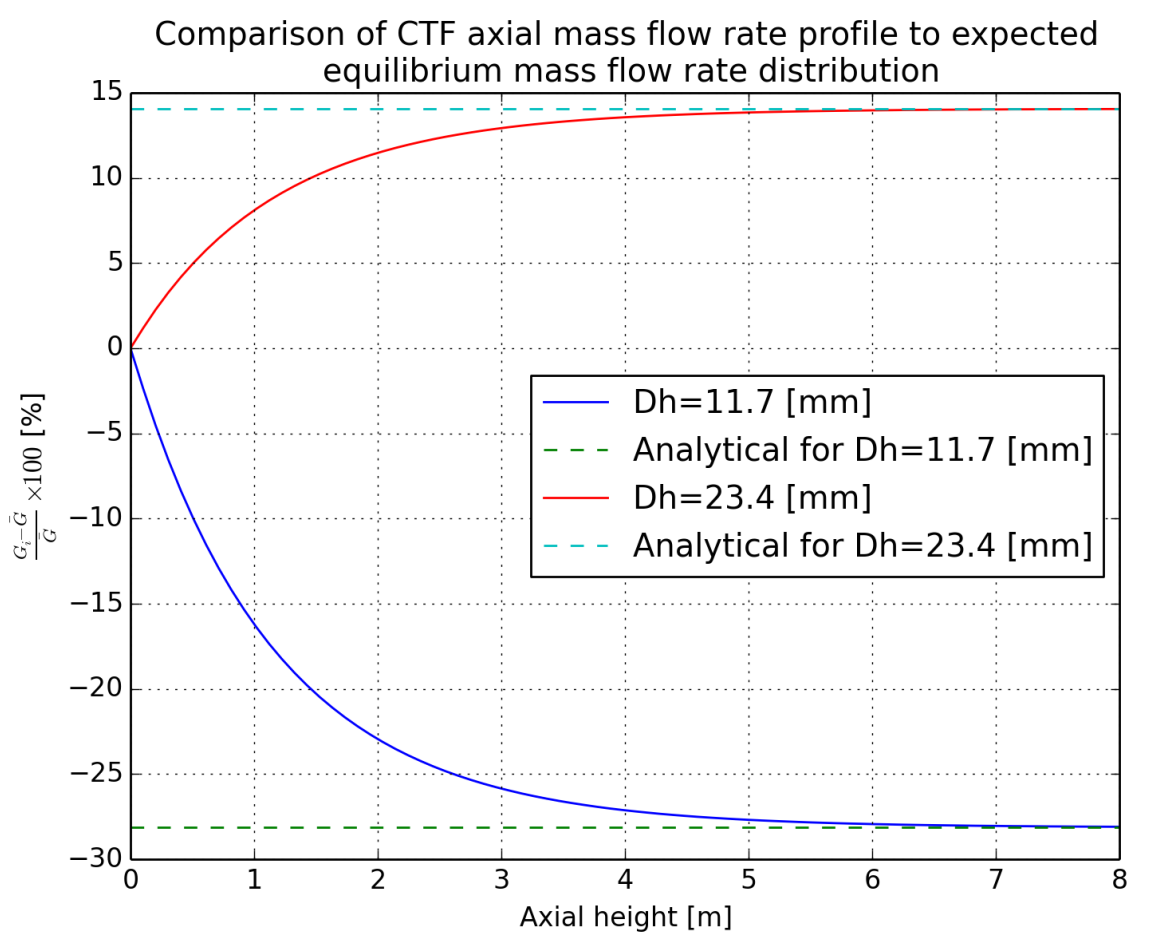

Figure 2. CTF-predicted axial mass flux distribution in two-channel system compared with analytical solution.

\subsection{Turbulent Mixing Verification}

Problem Description The problem consists of two channels connected by a gap. Because the CTF model for turbulent mixing is gradient-driven, it is necessary to make a gradient in either energy or momentum. Because there is no net transfer of mass due to turbulent mixing in single phase, unheated flows, it is not possible to analyze mass transfer in this case. Forming an analytical solution requires us to form and solve the relevant governing equations for the system.

If we choose to look at turbulent mixing of momentum, we will need to set velocity of one channel higher than the other. The result will be migration of velocity due to pressure-driven directed cross-flow (due to higher frictional pressure drop in the high velocity channel) as well as turbulent mixing of the momentum. We wish to verify that the turbulent mixing model works as expected without interference from other effects. We can disable the friction model to stop the pressure-driven directed cross-flow. However, because the axial velocity profile will not be constant in the channel, the convective terms of the momentum equation cannot be eliminated, which requires a complicated solution of the equations.

The energy equation can be solved much more easily as long as we disable the temperaturedependent density in CTF. With this disabled, the velocity profile will be constant, as the turbulent mixing model for energy does not actually move mass from one channel to another; it captures the effect of mixing on the migration of energy from one channel to the other.

This problem is a modification of Example 6-1 in Todreas and Kazimi Volume II [5]. The problem in the textbook uses the concept of tracer dyes to demonstrate mixing. In place of looking at mixing of a dye, the mixing of enthalpy is observed in this problem.

The design of the system is shown in Figure 3. The geometry of the two channels is identical in this case, which should eliminate any pressure-driven directed cross-flow. Channel geometry is based on typical PWR rod-lattice geometry. To activate the turbulent-mixing model, the temperature of one channel is raised $10^{\circ} \mathrm{C}$ over the second channel. The "vuq-param.txt" file is used to set a constant liquid density in the system. 


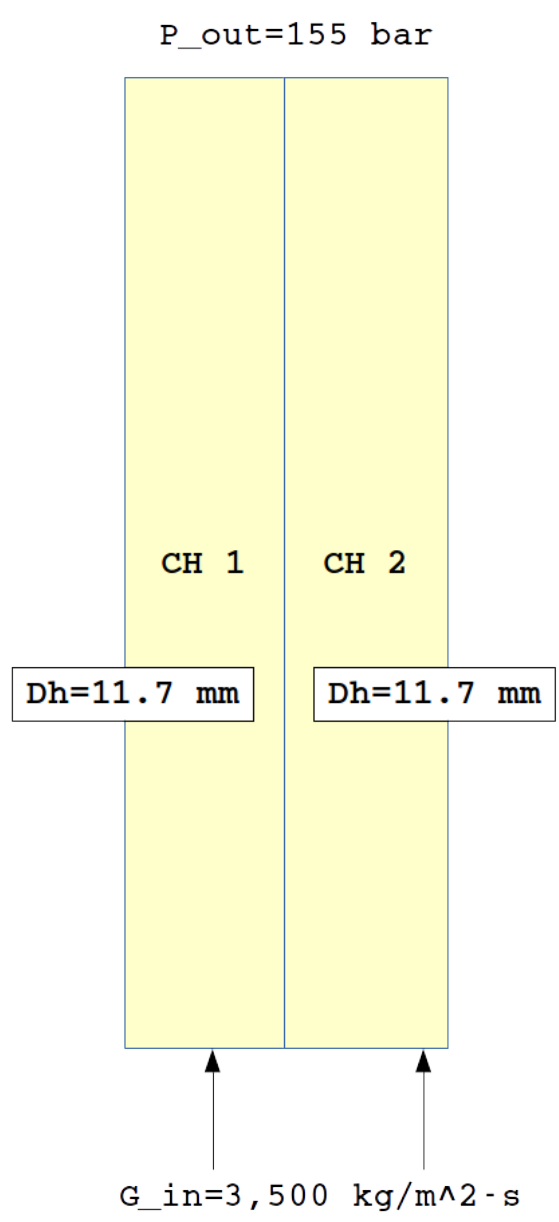

Figure 3. Model of problem for testing single-phase turbulent mixing of enthalpy. 
For this case, we can set up an energy equation for each channel. The CTF energy equation is as follows:

$$
\begin{gathered}
\frac{\partial}{\partial t}\left(\alpha_{k} \rho_{k} h_{k}\right)+\frac{\partial}{\partial x}\left(\alpha_{k} \rho_{k} h_{k} u_{k}\right)+\frac{\partial}{\partial y}\left(\alpha_{k} \rho_{k} h_{k} v_{k}\right)+\frac{\partial}{\partial z}\left(\alpha_{k} \rho_{k} h_{k} w_{k}\right)= \\
\sum_{\text {gap=1 }}^{N G A P} q_{k, \text { gap }}^{T^{\prime \prime \prime}}+\Gamma^{\prime \prime \prime} h+q_{w k}^{\prime \prime \prime}+\alpha_{k} \frac{\partial P}{\partial t}
\end{gathered}
$$

The left-hand side terms include: 1) time-change of energy, 2) axial $(x)$ advection of energy, 3) lateral $(y)$ advection of energy, and 4) lateral $(z)$ advection of energy. The terms, $\alpha, \rho, h$, and $u$, represent the volume fraction, density, enthalpy, and velocity, respectively. The subscript, $k$, indicates the field; liquid, vapor, or droplet. The right-hand side terms include: 1) turbulent mixing of energy (lateral direction only), 2) the implicit heat transfer, 3) energy entering the volume from the wall, and 4) the pressure-work on the volume over time. The term, $\Gamma^{\prime \prime \prime}$ represents the volumetric evaporation rate (transfer of mass from the liquid phase to the vapor phase).

We can make the following assumptions about this case:

1. The case is steady-state, eliminating the transient change in energy and the pressure work term.

2. The case is single-phase, eliminating all $k$ phase subscripts, void fractions, and the mass transfer term.

3. The case is unheated.

4. Because the case is set up so there is no lateral directed cross-flow, only the axial convective term remains.

The simplified equation, with $x$ being the axial direction, becomes

$$
\frac{\partial}{\partial x}(\rho h u)=q_{y}^{T^{\prime \prime \prime}}
$$

The density and velocity can be removed from the derivative because they are constants in the solution. This is applied directly to our problem of interest by formulating it for each channel in the model. Substituting the lateral transport of enthalpy due to turbulent mixing $\left(q_{y}^{T}\right)$ with the CTF form of the model yields the following set of equations:

$$
\begin{aligned}
& \dot{m}_{1} \frac{d}{d x} h_{1}+W_{1 \rightarrow 2}^{\prime}\left(h_{1}-h_{2}\right)=0 \\
& \dot{m}_{2} \frac{d}{d x} h_{2}-W_{1 \rightarrow 2}^{\prime}\left(h_{1}-h_{2}\right)=0
\end{aligned}
$$

Each equation is multiplied by the cross-sectional area to convert the velocity to mass flow rate and the volumetric mixing rate to a linear one. The $W_{1 \rightarrow 2}^{\prime}$ term represents the mixing rate of energy from Channel 1 to Channel 2. It has units of $\mathrm{kg} \mathrm{m}^{-1} \mathrm{~s}^{-1}$ and is defined as

$$
W_{1 \rightarrow 2}^{\prime}=\beta S_{12} \bar{G} .
$$

The $\beta$ term is the turbulent mixing coefficient; it is the "tuning parameter" for the mixing model. Physically, it is a non-dimensional coefficient that represents the ratio of the lateral mass flux due to mixing to the axial mass flux. The other terms, $S_{12}$ and $\bar{G}$, are the gap width between Channels 1 and 2 (in $\mathrm{m}$ ) and the area-weighted average mass flux between the two channels (in $\mathrm{kg} \mathrm{m}^{-2} \mathrm{~s}^{-1}$ ). For this case, we set $\beta$ to a "typical" value of 0.0035 , the gap thickness based on problem geometry $(0.003 \mathrm{~m})$, and the mass flux based on problem operating conditions.

Returning to Equations 16 and 17, we can solve the enthalpy distribution in a channel by relating the enthalpy in the two channels at any axial level, as follows: 


$$
\dot{m}_{1} h_{1}+\dot{m}_{2} h_{2}=\dot{m}_{1} h_{1, \text { in }}+\dot{m}_{2} h_{2, \text { in }}
$$

Because the mass flow rates in the channels are identical, this term cancels out and we are left with a relationship between the enthalpy in Channels 1 and 2 and the known inlet enthalpy boundary conditions. This is substituted into Equation 16 to develop a first-order, linear, ordinary differential equation that describes the enthalpy profile in Channel 2:

$$
\frac{d}{d x} h_{2}+\frac{2 W_{1 \rightarrow 2}^{\prime}}{\dot{m}} h_{2}-\frac{W_{1 \rightarrow 2}^{\prime}}{\dot{m}}\left(h_{1, \text { in }}+h_{2, \text { in }}\right)=0
$$

The solution of this equation is

$$
h_{2}=\frac{1}{2}\left(h_{1, \text { in }}+h_{2, \text { in }}\right)+C \exp \left(\frac{-2 W_{1 \rightarrow 2}^{\prime}}{\dot{m}} x\right) .
$$

Using the inlet enthalpy boundary conditions, the value of the constant, $C$ is determined. The same process is repeated for the first channel, leading to the following final solutions for enthalpy distribution in the system due to turbulent mixing.

$$
\begin{aligned}
& h_{1}=\frac{1}{2}\left(h_{1, \text { in }}+h_{2, \text { in }}\right)-\frac{1}{2}\left(h_{2, \text { in }}-h_{1, \text { in }}\right) \exp \left(\frac{-2 W_{1 \rightarrow 2}^{\prime}}{\dot{m}} x\right) \\
& h_{2}=\frac{1}{2}\left(h_{1, \text { in }}+h_{2, \text { in }}\right)+\frac{1}{2}\left(h_{2, \text { in }}-h_{1, \text { in }}\right) \exp \left(\frac{-2 W_{1 \rightarrow 2}^{\prime}}{\dot{m}} x\right)
\end{aligned}
$$

CTF Model Description The CTF input deck is set up from Figure 3. Axial meshing is set to $2.54 \mathrm{~cm}$. The gap thickness is set to $0.003 \mathrm{~m}$ and its length is set to $0.0126 \mathrm{~m}$, which would result from a PWR lattice with $12.6 \mathrm{~mm}$ pitch and $9.5 \mathrm{~mm}$ rod outside diameter. The turbulent mixing model is set so that a single-phase turbulent-mixing parameter, $\beta$, could be set equal to the value used in the analytical solution. The liquid density is set to a constant value of $700 \mathrm{~kg} \mathrm{~m}^{-3}$. Note, however, that density does not appear in the analytical solution and, thus, has no impact on the $\mathrm{CTF}$ results in terms of turbulent mixing of enthalpy.

Discussion of Results The case is run to steady-state in CTF and the axial enthalpy profile is extracted for each channel. Figure 4 shows results for the two channels compared with the analytical solution that is calculated above. The figure demonstrates excellent agreement between code and expected results.

As noted above, the turbulent mixing model is defined so that it predicts net transfer of the parameter of interest due to turbulent exchange between channels. This transfer may or may not include mass transfer. For a single-phase, unheated case, turbulent exchange leads to zero net mass transfer because each unit of liquid volume that moves from Channel 1 to 2 is replaced with an equal volume that moves from Channel 2 to 1 . The exchange does cause enthalpy to be moved, as is observed in Figure 4. A check is made on the axial mass flow rate profile in each of the channels in Figure 5.

It is noted that there is a slight discrepancy in the axial mass flow rate profile. Ideally, the normalized mass flux should be zero everywhere; however, the plot indicates that it varies by as much as $0.13 \%$ from the average mass flux. This discrepancy is further investigated by disabling the turbulent-mixing and void drift, leading to the flow redistribution shown in Figure 6.

The magnitude of the flow redistribution is small; however, results indicate that something in the solution is causing a slight drift in mass flux despite the channels being geometrically identical. 


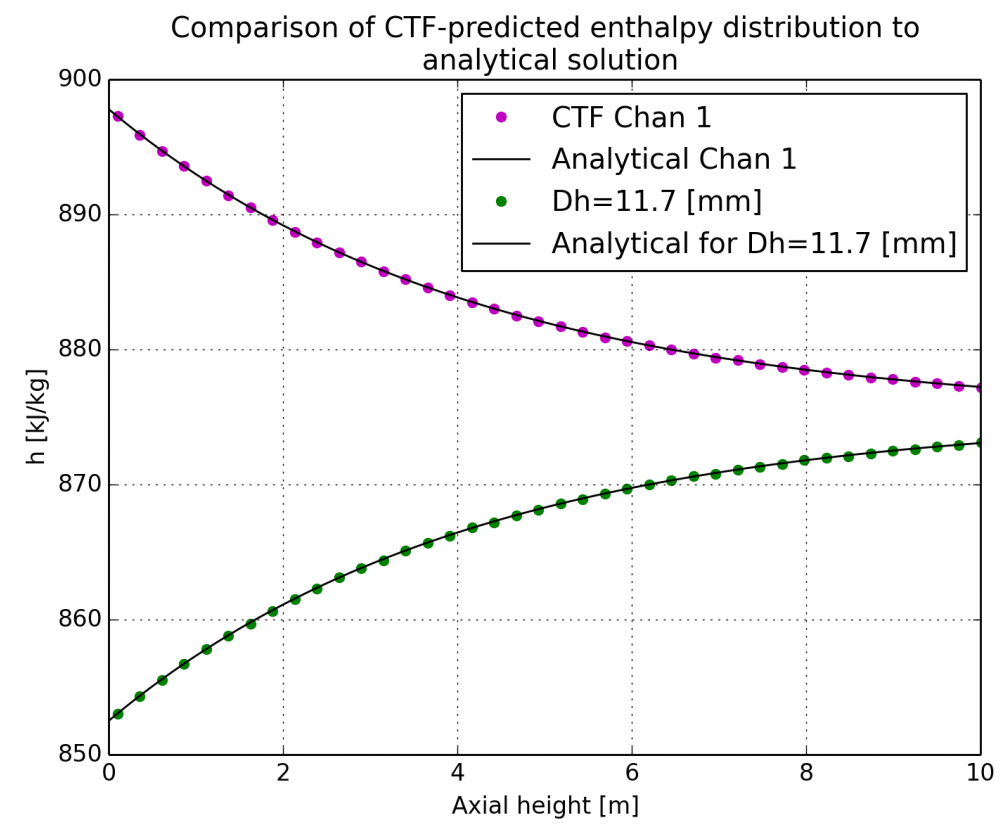

Figure 4. Comparison of CTF-predicted liquid enthalpy in channels and the analytical solution.

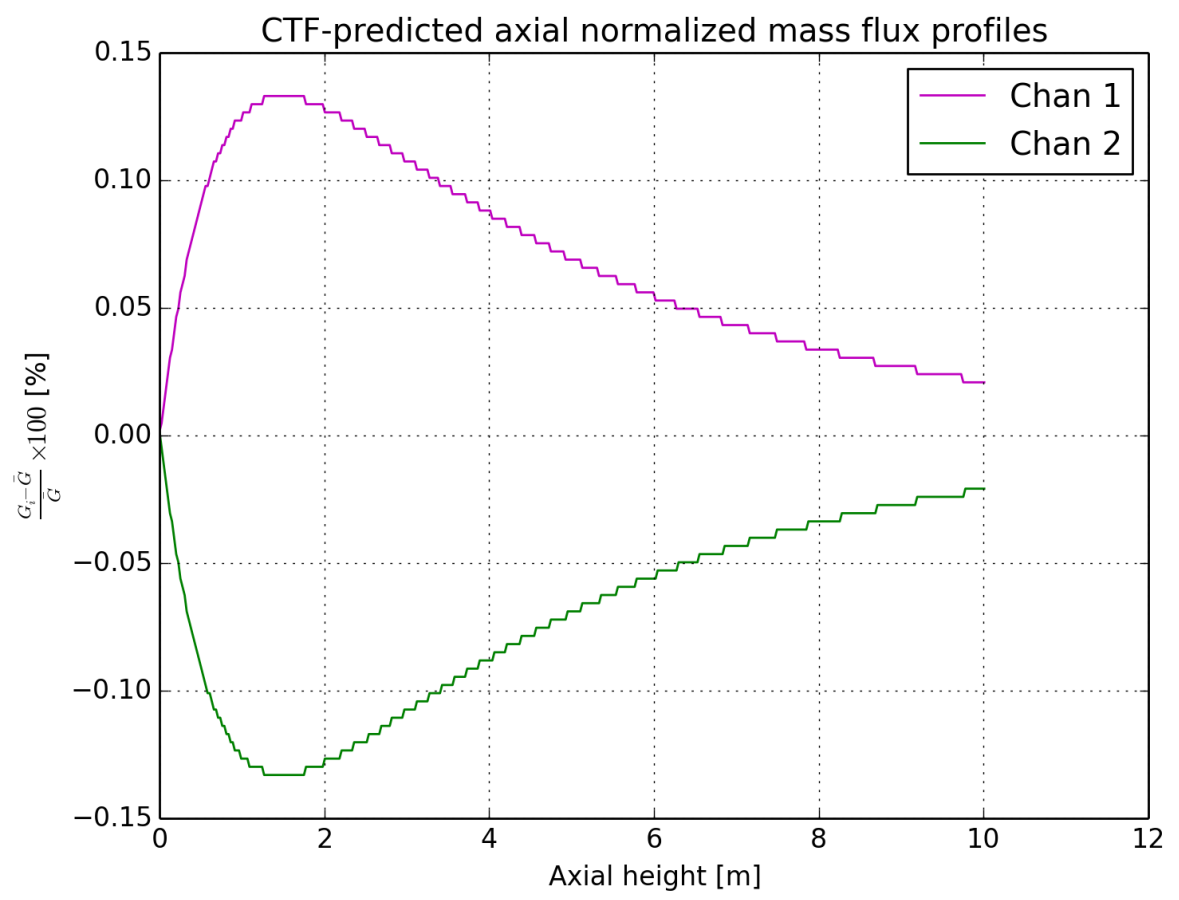

Figure 5. CTF-predicted axial mass flow rate profile in the channels. 


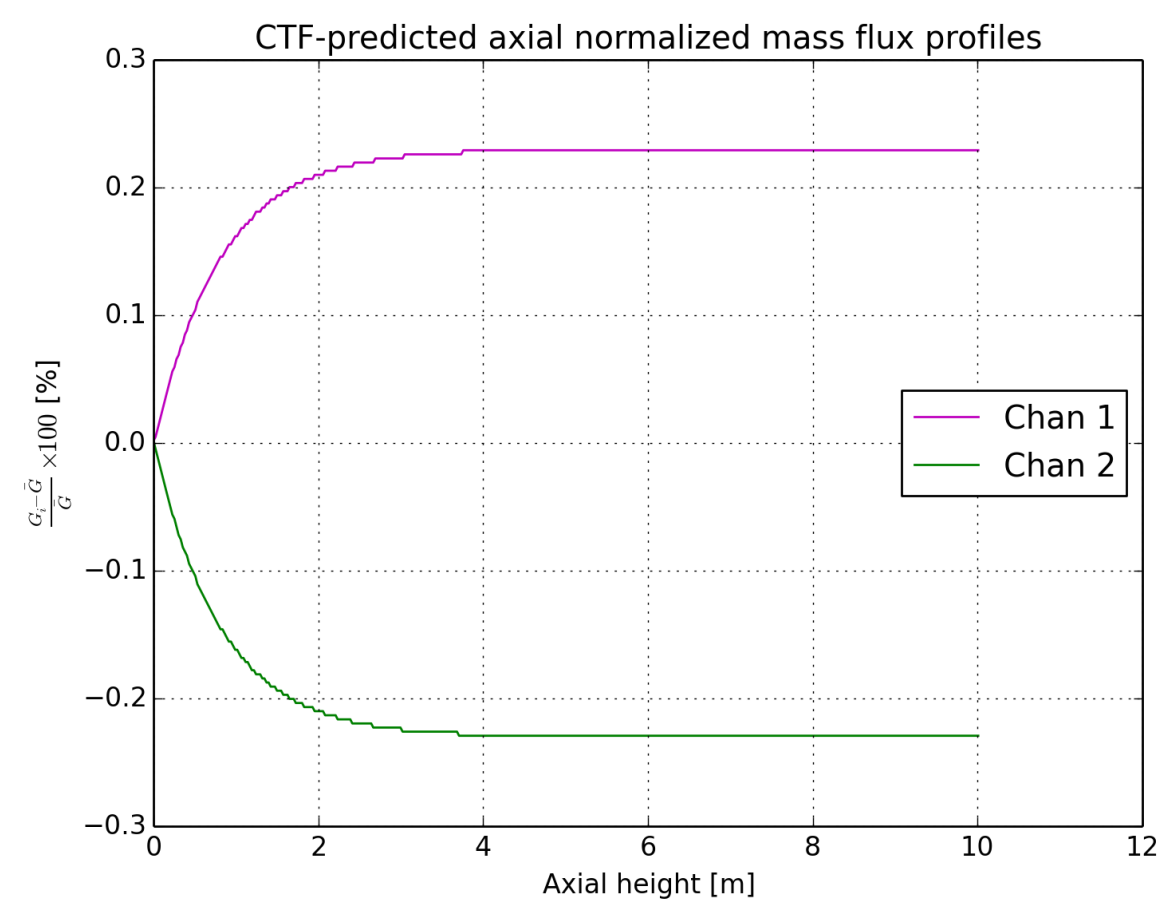

Figure 6. CTF-predicted axial mass flow rate profile in the channels with turbulentmixing disabled.

\section{$2.32 \times 3$ Validation}

Facility Description The $2 \times 3$ facility is an air/water facility that was operated at Kumamoto University in the early 2000s in order to quantify effects of mixing and void drift [6]. The test section is uniquely designed so that there are only two subchannel types - side and inner-leaving out the additional complexity of the corner type subchannel. Figure 7 shows the cross-section of the facility and provides geometric information. The assembly geometry is larger than a typical BWR lattice due to the lower density (and thus larger bubble sizes) of air at atmospheric pressure.

Figure 8 shows an axial schematic of the facility. The test section has four axial sections: 1) an entry section, where side and inner channels are separated by a physical barrier; 2) a tracer injection section, where each individual channel is physically separated from one another; 3) a mixing section, where all channel are connected and lateral cross-flow is allowed to take place; and 4) a discharge section, where groups of channels are partitioned and flow is sent to measurement devices.

Because the mixing section is short $(2.25 \mathrm{~m})$, the inlet flow of individual subchannel types is adjusted in the experiment so that flow in the mixing section is in mechanical equilibrium throughout the entire length. In this way, the test eliminates the effects of flow redistribution due to geometry differences between side and inner channels.

The facility is used for two different types of tests: single- and two-phase mixing tests [6] and void drift tests [7]. The single-phase mixing tests are the focus of this study, as difficulty is encountered in modeling low-pressure air/water tests using CTF, as will be noted in discussing the $2 \times 2$ facility results.

The mixing tests made use of tracer dyes in the facility. The tracer dyes include Acid orange II for the water phase and methane for the gas phase. They are injected into one of the inner subchannels in the tracer injection section and collected and measured at three axial elevations in the mixing section. Measurements are taken by gas chromatograph for the gas phase and spectrophotometer for the liquid phase.

The author derived a tracer-dye conservation equation for each subchannel and used this system 


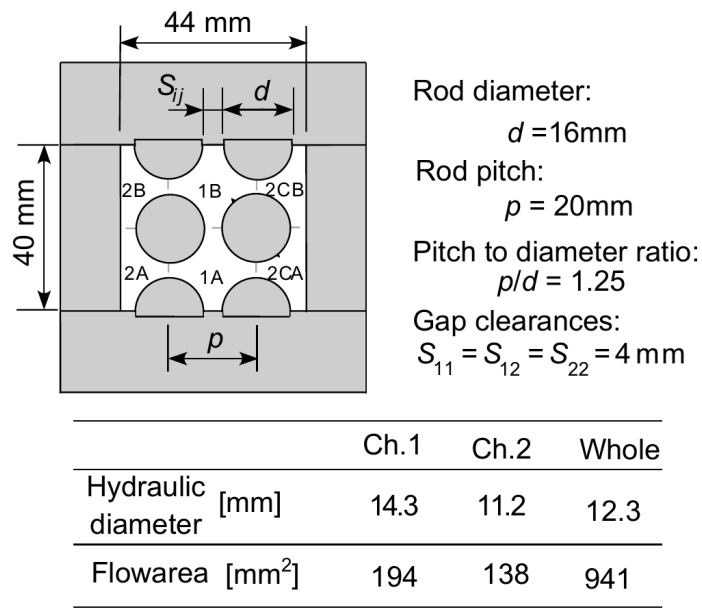

Figure 7. Cross-sectional diagram of the $2 \times 3$ facility and relevant geometric information (reprinted from M. Sadatomi et al. "Single- and Two-Phase Turbulent Mixing Rate between Adjacent Subchanels in a Vertical $2 \times 3$ Rod Array Channel". In International Journal of Multiphase Flow 30 (2004), pp. 481-498.

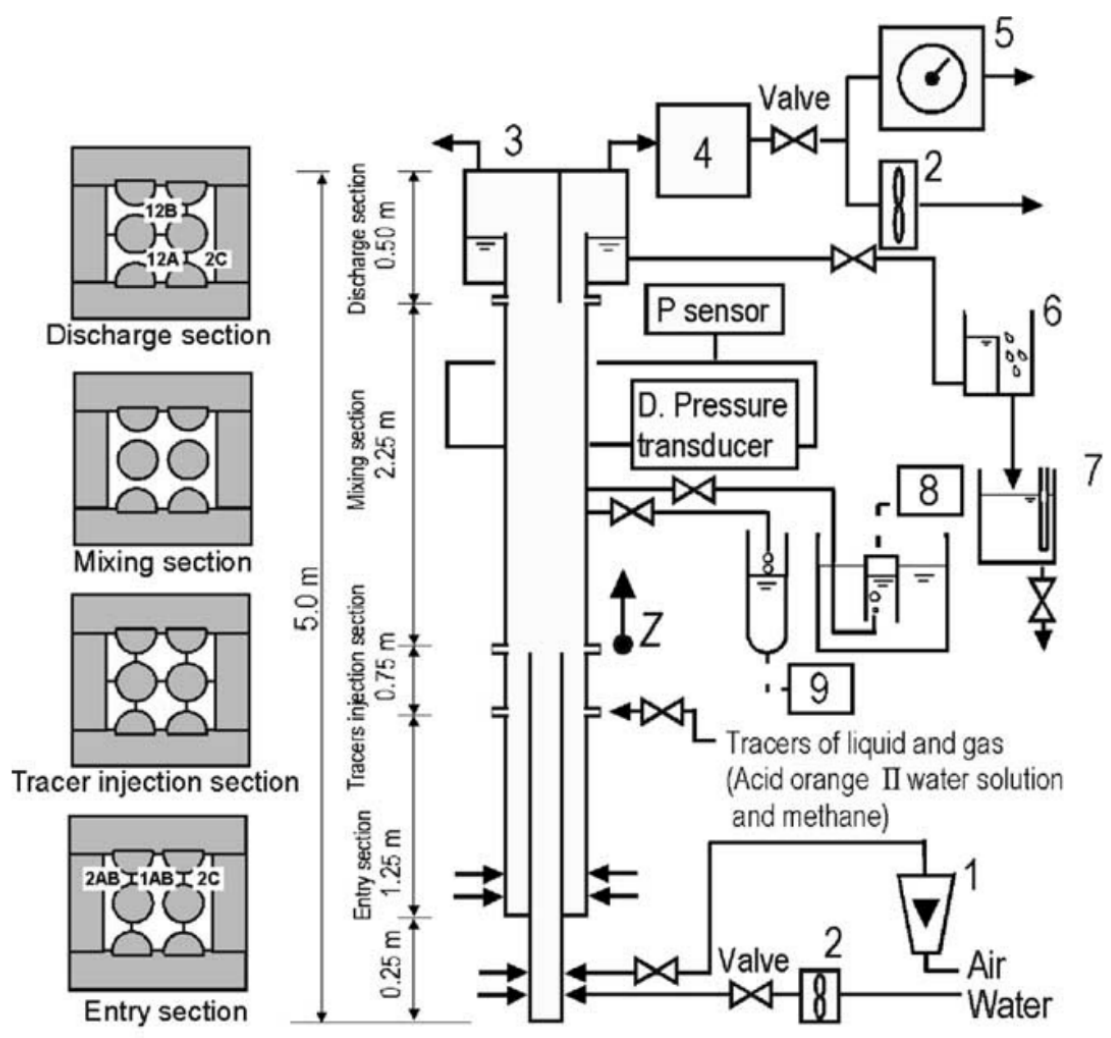

Figure 8. Side-view schematic of $2 \times 3$ facility with visualization of channel partitioning in different axial sections (reprinted from M. Sadatomi et al. "Single- and Two-Phase Turbulent Mixing Rate between Adjacent Subchanels in a Vertical $2 \times 3$ Rod Array Channel". In International Journal of Multiphase Flow 30 (2004), pp. 481-498. 
of equations to derive the channel mixing term, $w_{i j}^{\prime}$, as a function of tracer dye concentration. Flow conditions for the mixing tests are shown in Figure 9 as liquid and vapor superficial velocity. Only the single-phase tests are modeled in this study, which includes four data points. The system is run at room temperature and atmospheric pressure.

CTF Model Description Flow area and wetted perimeter are directly taken from the values of Figure 7. An axial mesh size of $2.54 \mathrm{~cm}$ is employed, and only the $2.25 \mathrm{~m}$ mixing section is modeled. Because the experimenters set the inlet flow to equal the equilibrium distribution, a similar approach is used in setting the inlet flow rate in CTF. First, the total injection mass flow rate is determined using the CTF-predicted liquid density, facility flow area, and liquid velocity specified in the experiment. CTF is run, the outlet flow distribution is obtained, and this is used as the inlet distribution for the next simulation. This process is repeated until cross-flow ceases throughout the facility.

The friction correlation will drive the flow distribution, as shown in Section 2.1. The author indicates that the Sadatomi friction factor correlation [8] leads to the best agreement with the measured flow distribution; however, its complexity makes it difficult to enter into CTF without significant code changes. The author also shows results of the Blasius equation, which seems to also perform well. Therefore, the Blasius correlation (Equation 24) is used as a first step in this study; however, the CTF friction correlation (Equation 25), which is a default model in CTF, is also tested.

$$
\begin{gathered}
f=0.316 R e^{-0.25} \\
f=0.204 R e^{-0.2}
\end{gathered}
$$

The single-phase turbulent mixing coefficient is varied during this study to investigate its impact on mixing results. The inlet temperature is set to $24^{\circ} \mathrm{C}$ and the outlet pressure is set to 1.01325 bar.

Discussion of Results Results are shown as the nondimensional mixing rate, $W_{i j}^{\prime} / \mu$, vs. the two-channel Reynolds number. The two-channel Reynolds number is calculated as follows:

$$
R e_{i j}=\frac{\rho u_{i j} D h_{i j}}{\mu}
$$

Where the average two-channel velocity, $u_{i j}$, is an area weighted average of the velocities in the two adjacent channels, $i$ and $j$. The hydraulic diameter, $D h_{i j}$, is the hydraulic diameter of the two-channel system, $\rho$ is density, and $\mu$ is dynamic viscosity. This is the way in which the author presents mixing results. A mixing rate is given for each unique channel connection, which includes: 1) inner-to-inner, 2) side-to-inner, and 3) side-to-side for each of the four single phase tests. Figure 10 shows the CTF results compared with the experimental results using the Rogers and Rosehart correlation [2] to predict single-phase mixing. The Blasius friction factor correlation is used in this case.

Two types of data points are shown in the plot: pluses represent the CTF predictions and circles represent the experimental measurements. There are three colors of the data points: red represents the inner-to-inner connection, green represents the side-to-side connection, and blue represents the side-to-inner connection. Ideally, a "plus" and a "circle" data point should sit in a vertical column; this would mean that the $i j$ Reynolds number of CTF matches the experimental value exactly. Looking at the figure, it is evident this is not the case. The CTF-predicted Reynolds number tends to be higher than its experimental counterpart in every case.

Likely, there are some differences in steam properties and inlet mass flow rate that lead to this discrepancy. However, it is evident that there is a near-linear trend for nondimensional mixing with respect to Reynolds number that we can use for comparison. The results of Figure 10 indicates that the Rogers and Rosehart correlation over-predicts the mixing rate observed in this facility substantially.

The study is re-run with a user-set, constant single-phase mixing coefficient of 0.004. A mixingcoefficient optimization study done using the CE $5 \times 5$ facility [9] (an electrically heated rod bundle 


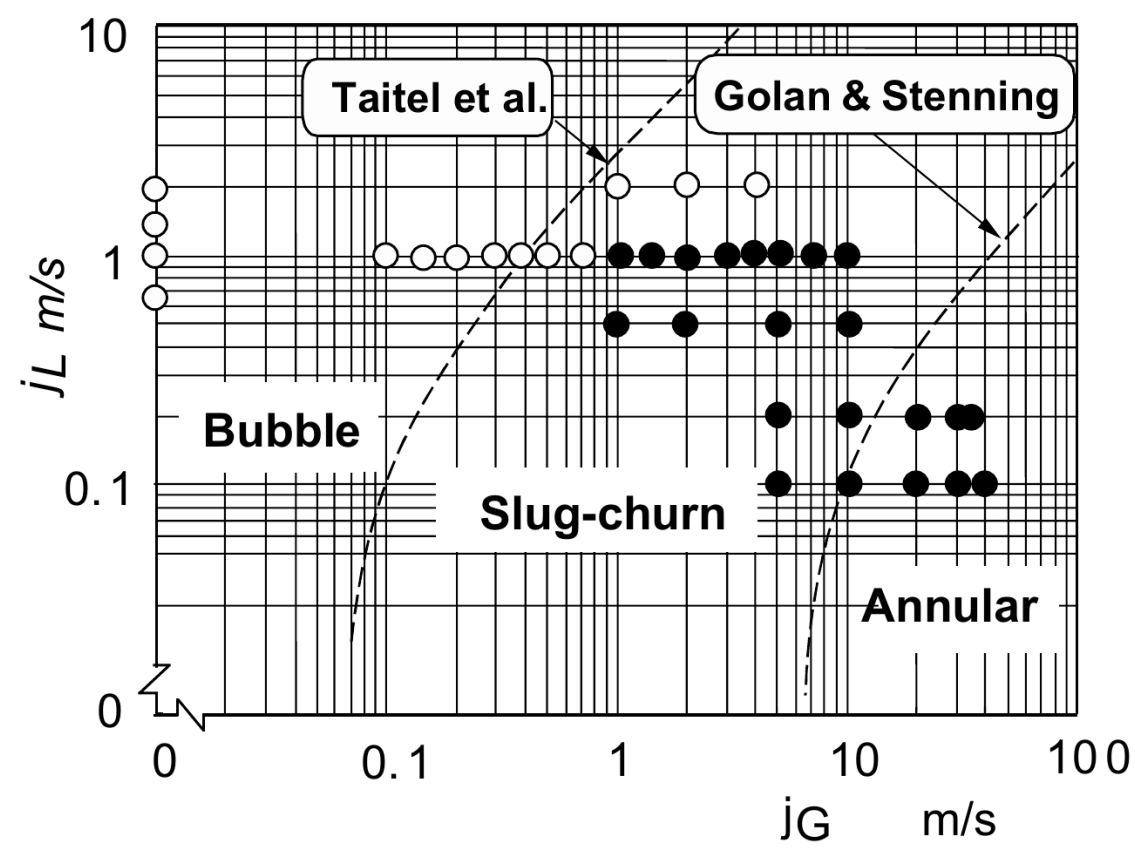

Figure 9. Operating conditions for the $2 \times 3$ facility (reprinted from M. Sadatomi et al. "Single- and Two-Phase Turbulent Mixing Rate between Adjacent Subchanels in a Vertical $2 \times 3$ Rod Array Channel". In International Journal of Multiphase Flow 30 (2004), pp. 481-498.

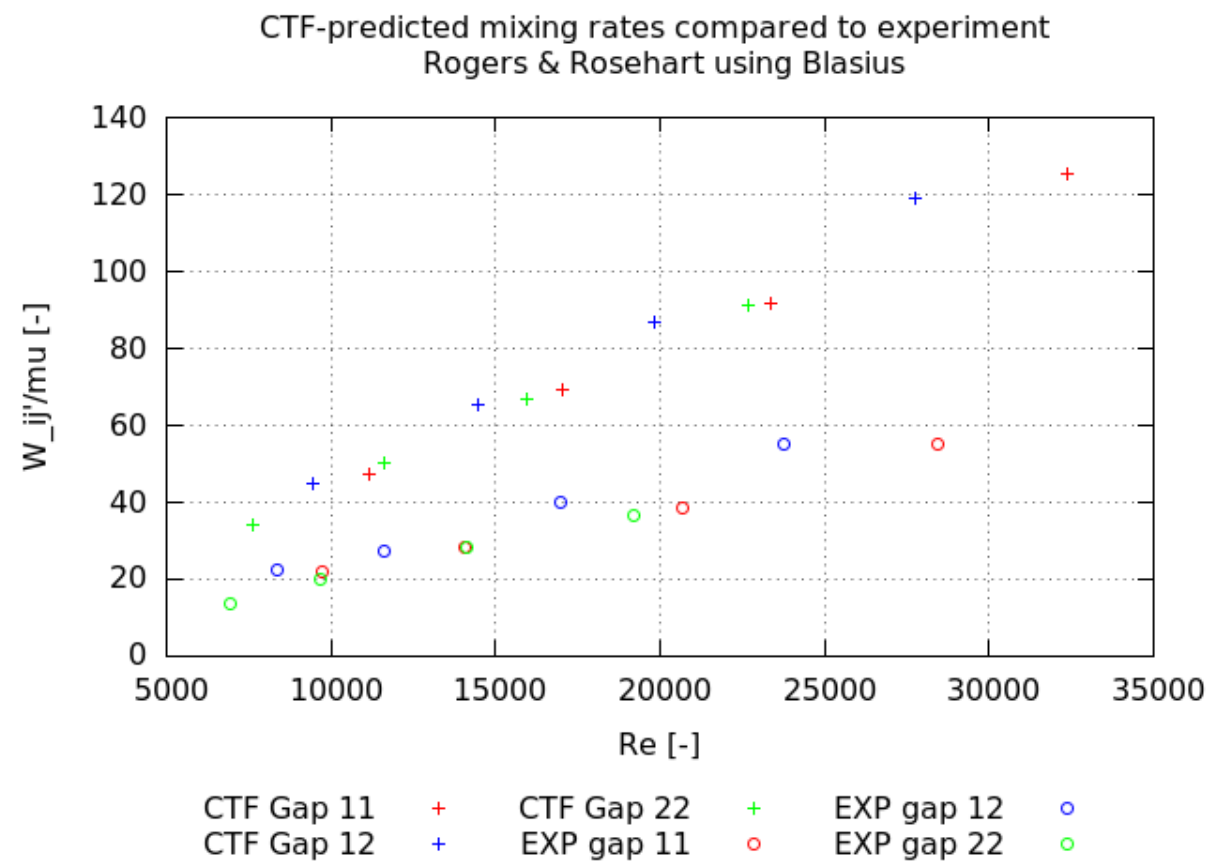

Figure 10. Comparison of CTF-predicted mixing rates and experimental measured rates from $2 \times 3$ facility using Rogers and Rosehart for $\beta$ and the Blasius friction correlation. 
representative of PWR geometry) found that a value of 0.0044 was optimum for that configuration [10], so 0.004 is considered to be a lower bounding value. Figure 11 shows the results of changing the mixing coefficient to 0.004 .

Results indicate that this mixing coefficient underpredicts the mixing in the facility. As it turns out, a mixing coefficient of about 0.007 tends to lead to the best agreement, as shown in Figure 12. The choice of friction factor correlation has little impact on the predicted mixing rates. Figure 13 shows the results using $\beta=0.004$ with the friction factor correlation set to the CTF correlation instead of the Blasius correlation.

It is important to note that the mixing coefficient is simply a tuning parameter that will be dependent on the actual geometry of the facility being modeled. This facility is a square lattice, but the geometry is much larger than typical PWR or BWR rod-lattice geometry. This study is useful for showing that CTF is capable of predicting the correct mixing rate if $\beta$ is tuned correctly to the facility. Furthermore, it offers a range of values from which to select the mixing coefficient.

The physical relevance of the mixing rate is not immediately obvious. It is better to observe the impact of the term on simulation parameters that affect the solution. The CASL Problem 7 challenge problem (quarter symmetry model of Watts Bar Unit 1) is modeled using a power distribution from a coupled MPACT/CTF solution of the facility. The mixing coefficient is changed from 0.0035 to 0.05 , with 0.05 being much greater than the value predicted by Rogers and Rosehart. The impact of changing this parameter on local predicted liquid density is shown in Figure 14. The results are presented as density in a cell when $\beta$ is 0.05 minus density in the cell when $\beta$ is 0.0035 . This calculation is made in each of the roughly 500,000 computational cells of the model and presented in the figure. The results show that differences increase to a maximum at the outlet of the facility and reach as much as $0.01 \mathrm{~g} \mathrm{~cm}^{-3}$, which will have a small, but noticeable impact on reactivity in those locations.

\subsection{RPI $2 \times 2$ Validation}

Facility Description The intended purpose of this experiment (Air/Water Subchannel Measurements of the Equilibrium Quality and Mass Flux Distribution in a Rod Bundle) is to investigate the fully developed two-phase flow distribution in a $2 \times 2$ rod array test section. The test facility includes a $91.4 \mathrm{~cm}$ long unheated $2 \times 2$ rod bundle with an air/water mixture as the working fluid. With a bundle hydraulic diameter of $23.2 \mathrm{~mm}$, an length-to-diameter ratio of 39 is calculated, leading to an expected fully-developed flow condition at the bundle outlet.

Four $0.1397 \mathrm{~cm}$ thick 314 stainless steel tubes with $2.54 \mathrm{~cm}$ outside diameter are used to simulate the fuel rods. The wall thickness insured a vibration-free environment during the experiment, and a lower tie plate provides support for the rods. No spacer grids are used in this experiment. In order to realistically simulate boiling, two different techniques were used to distribute the air into the bundle inlet: a sinter sections technique and a mixing tee technique. An isokinetic sampling approach is used at the bundle outlet to measure liquid and vapor mass flow rates for each unique subchannel type (i.e., corner, side, and inner). The maximum experimental measurement uncertainty of exit mass flux is estimated to be $\pm 5 \%$. Further details of the facility are summarized in the technical report [3] as well as the CTF Validation Manual [4].

CTF Model Description Table 1 is used to set the channel flow areas in the CTF model. A $2.54 \mathrm{~cm}$ axial mesh is used. The pressure in Table 2 is used as the outlet pressure boundary condition. The inlet mass flowrate is set from Table 2 , and the inlet temperature is set to $21.1^{\circ} \mathrm{C}$, because the technical document specifies that the tests were run at room temperature.

Discussion of Results In this step of the validation, we are concerned only with correctly predicting the single-phase flow distribution. Therefore, only the two single-phase tests are modeled (Tests 1 and 2). The ideal flow distribution is determined similar to how it is in Section 2.1; the momentum equation is used to relate velocity in two individual subchannels, and the mass conservation equation is used to link all channels together. In this case, we have two unique momentum equations and one 


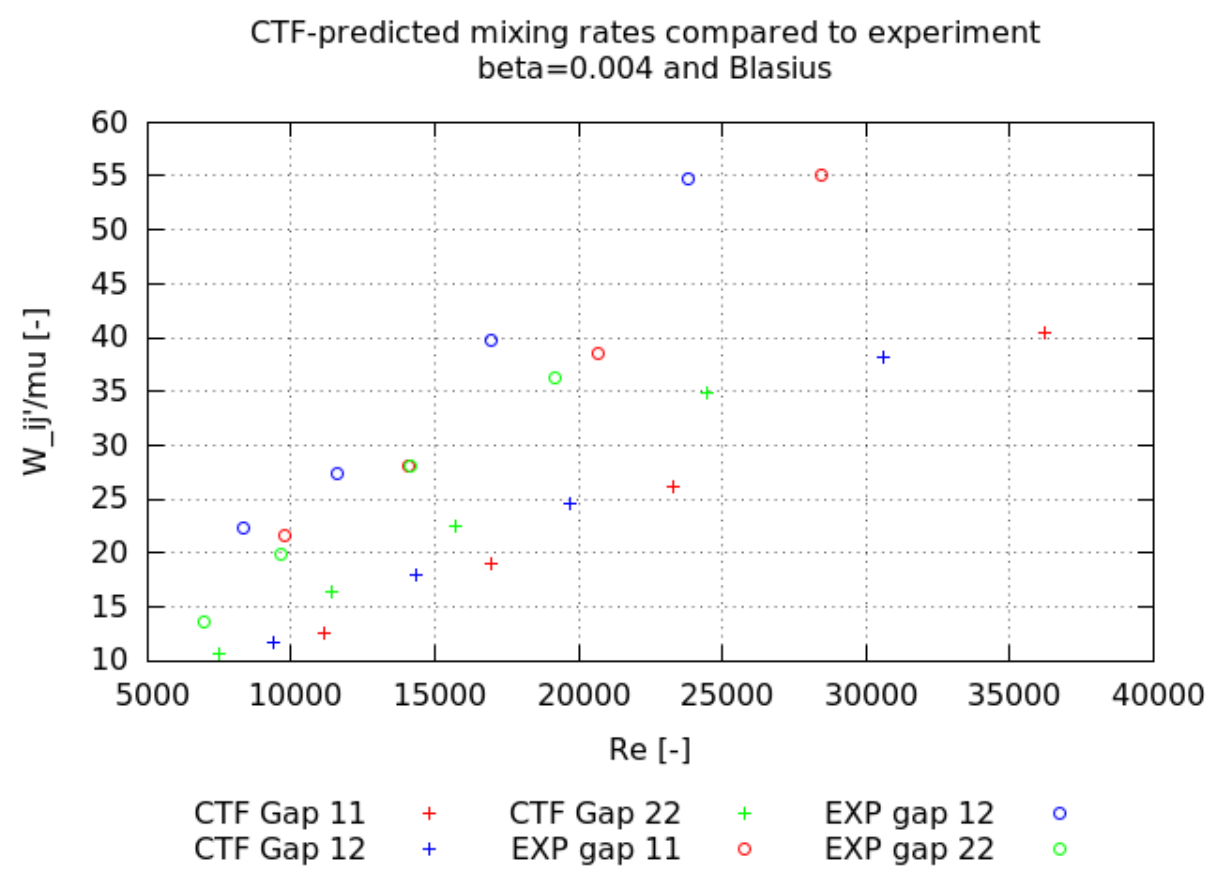

Figure 11. Comparison of CTF-predicted mixing rates and experimental measured rates from $2 \times 3$ facility using $\beta=0.004$ and the Blasius friction correlation.

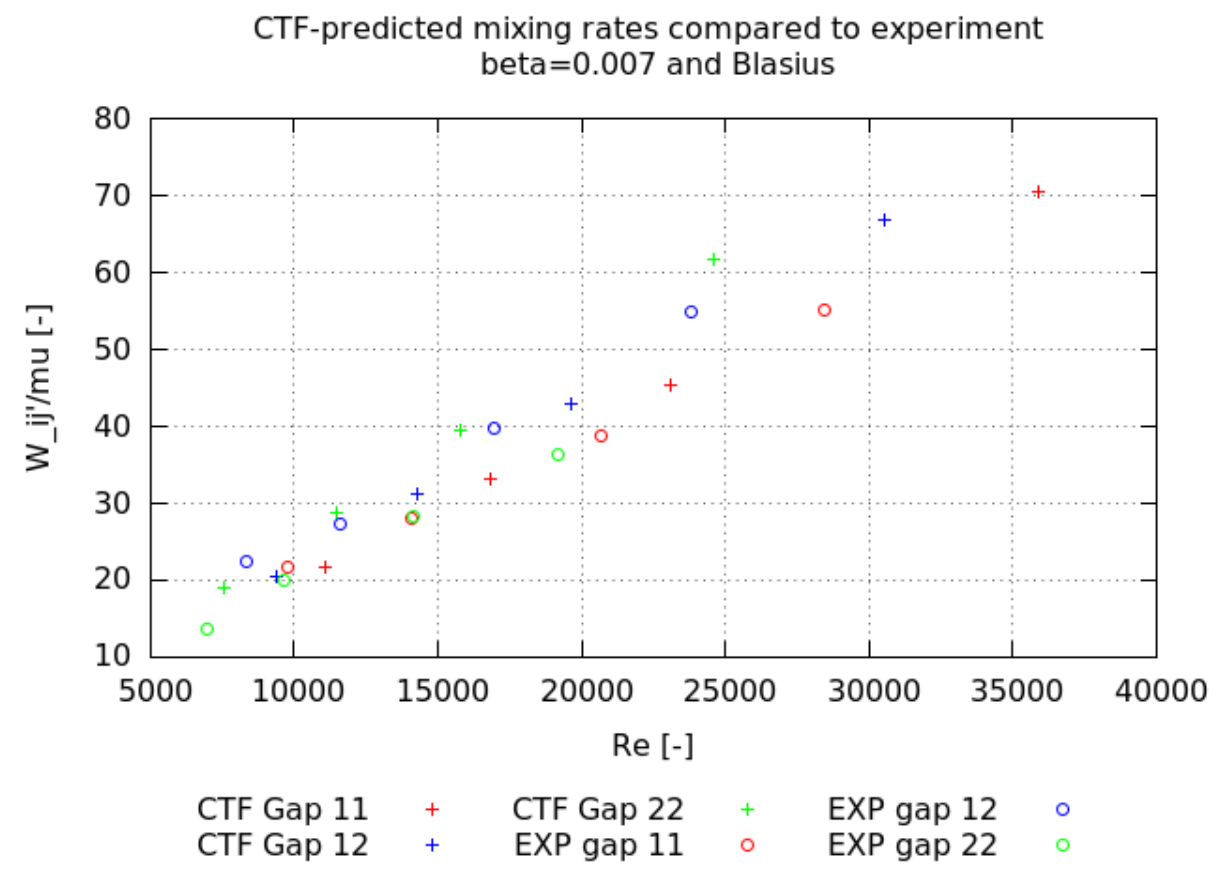

Figure 12. Comparison of CTF-predicted mixing rates and experimental measured rates from $2 \times 3$ facility using $\beta=0.007$ and the Blasius friction correlation. 


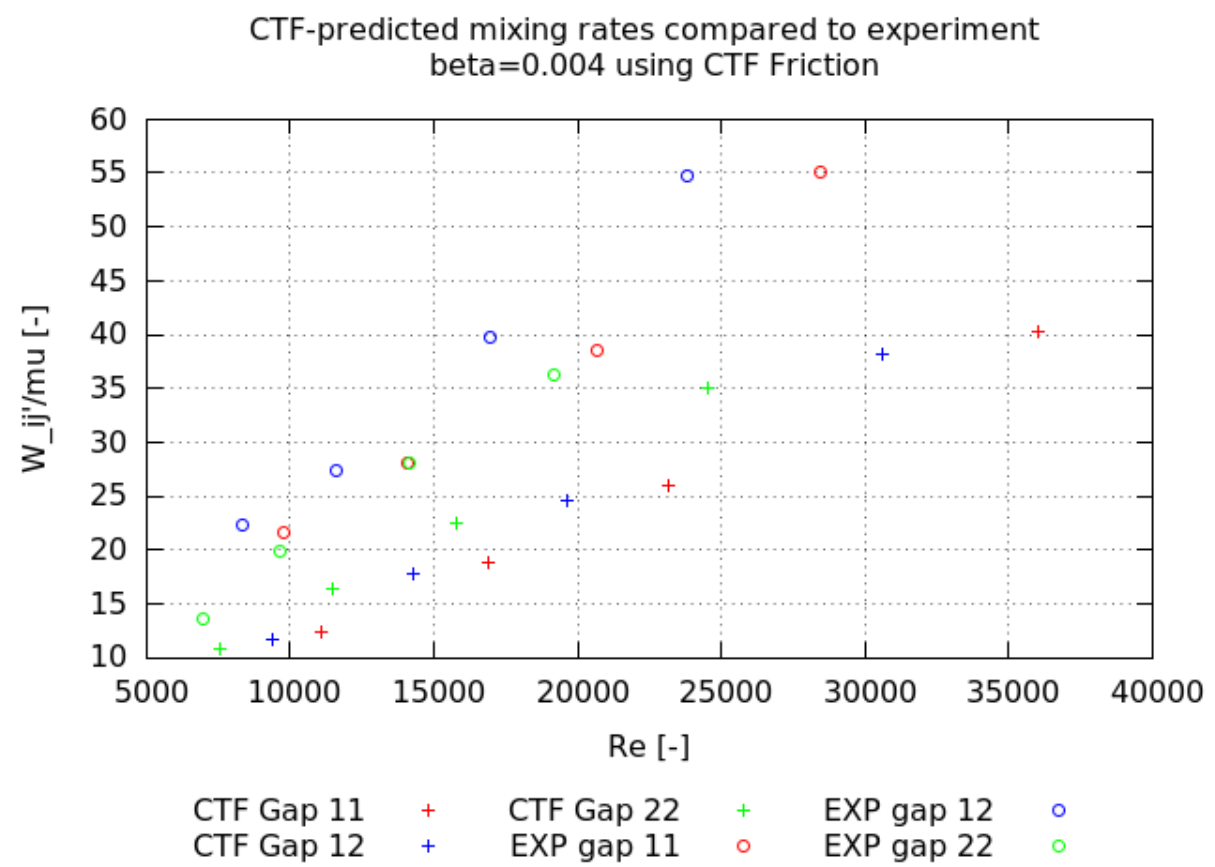

Figure 13. Comparison of CTF-predicted mixing rates and experimental measured rates from $2 \times 3$ facility using $\beta=0.007$ and the $\mathrm{CTF}$ friction correlation.

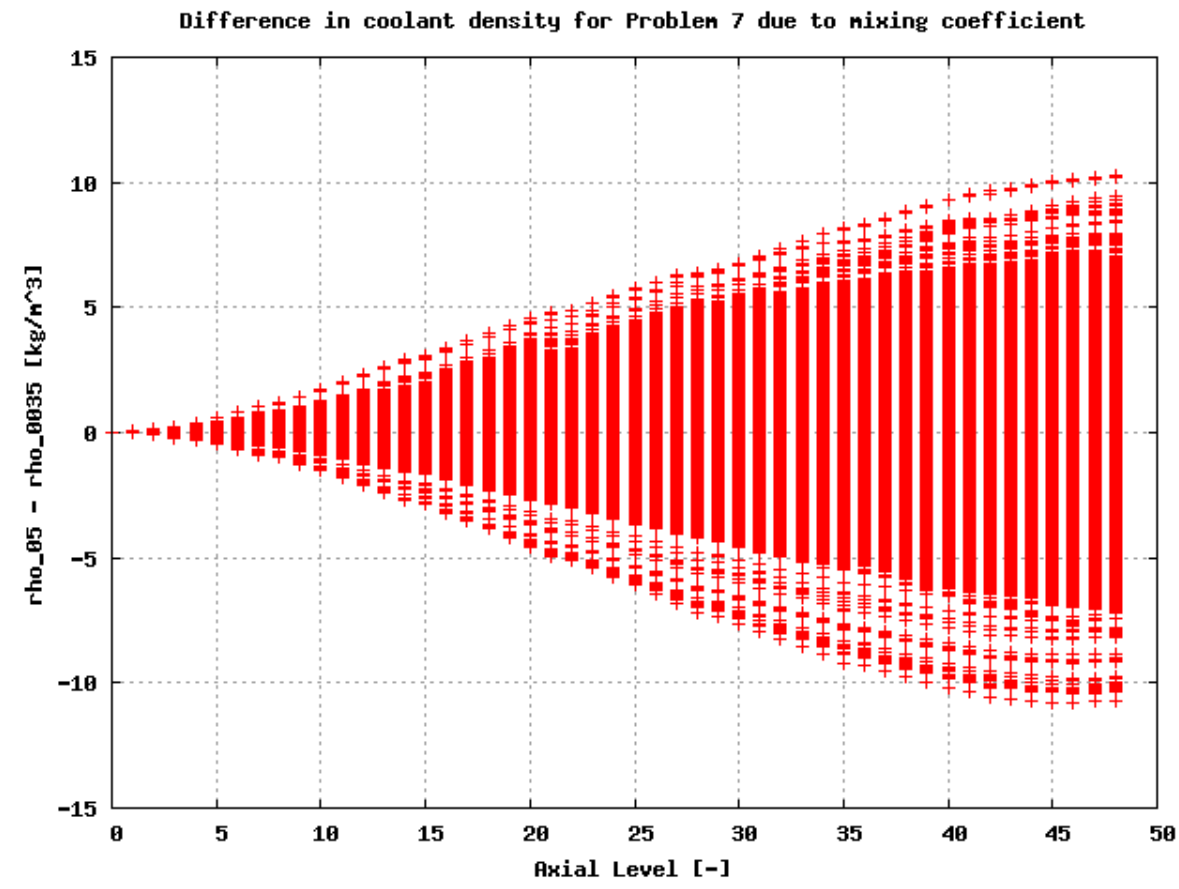

Figure 14. Variation of local liquid density in CTF simulation of Watts Bar Unit 1 when $\beta$ is changed from 0.0035 to 0.05 . 
Table 1. NUREG Subchannel Flow Dimensions

\begin{tabular}{lrr}
\hline Type & Flow Area $\left(i n^{2}\right)$ & Hydraulic Diameter (in) \\
\hline Corner & 2.08 & 1.59 \\
Side & 4.68 & 2.50 \\
Center & 7.13 & 3.57 \\
Total & 34.14 & 2.32 \\
\hline
\end{tabular}

Table 2. Experimental Operating Conditions

\begin{tabular}{lrrrr}
\hline Case & $\begin{array}{r}\mathrm{G} \cdot 10^{-6} \\
{\left[\mathrm{lbm} \mathrm{ft}^{-1} \mathrm{~h}^{-2}\right]}\end{array}$ & $\begin{array}{r}\langle\bar{\alpha}\rangle \\
{[\%]}\end{array}$ & $\begin{array}{r}\mathrm{P}_{i n} \\
{[\mathrm{psig}]}\end{array}$ & Mixing Technique \\
\hline 1 & 0.333 & 0.0 & 6.0 & \\
2 & 0.666 & 0.0 & 15.0 & \\
\hline 3 & 0.333 & 20.4 & 6.0 & \\
4 & 0.333 & 32.8 & 6.5 & \\
5 & 0.333 & 46.1 & 8.0 & Mixing Tee \\
6 & 0.666 & 23.6 & 15.5 & \\
7 & 0.666 & 37.5 & 19.0 & \\
8 & 0.666 & 52.7 & 26.0 & \\
\hline 9 & 0.333 & 20.4 & 6.0 & \\
10 & 0.333 & 32.8 & 6.3 & \\
11 & 0.333 & 46.1 & 8.0 & 4 \\
12 & 0.666 & 23.6 & 15.5 & \\
13 & 0.666 & 37.5 & 19.0 & \\
14 & 0.666 & 52.7 & 29.5 & \\
\hline
\end{tabular}


mass equation with three unknown variables. The coefficients for the CTF friction correlation are used in the momentum equation, leading to an exponent of $2 / 3$ on the ratio of hydraulic diameters.

$$
\begin{aligned}
\frac{u_{\text {corner }}}{u_{\text {side }}} & =\left(\frac{D h_{\text {corner }}}{D h_{\text {side }}}\right)^{2 / 3} \\
\frac{u_{\text {corner }}}{u_{\text {inner }}} & =\left(\frac{D h_{\text {corner }}}{D h_{\text {inner }}}\right)^{2 / 3} \\
\dot{m}_{\text {tot }} & =4 \rho u_{\text {corner }} A_{\text {corner }}+4 \rho u_{\text {side }} A_{\text {side }}+\rho u_{\text {inner }} A_{\text {inner }}
\end{aligned}
$$

Solving this system of equations gives an ideal flow split for each individual channel type:

$$
\begin{gathered}
\dot{m}_{\text {corner }}=\frac{\dot{m}_{\text {tot }}}{C}\left(\frac{D h_{\text {corner }}}{D h_{\text {inner }}}\right)^{2 / 3}\left(\frac{A_{\text {corner }}}{A_{\text {inner }}}\right) \\
\dot{m}_{\text {side }}=\frac{\dot{m}_{\text {tot }}}{C}\left(\frac{D h_{\text {side }}}{D h_{\text {inner }}}\right)^{2 / 3}\left(\frac{A_{\text {side }}}{A_{\text {inner }}}\right) \\
\dot{m}_{\text {inner }}=\frac{\dot{m}_{\text {tot }}}{C}, \text { where } \\
C=4\left(\frac{D h_{\text {corner }}}{D h_{\text {inner }}}\right)^{2 / 3} \frac{A_{\text {corner }}}{A_{\text {inner }}}+4\left(\frac{D h_{\text {side }}}{D h_{\text {inner }}}\right)^{2 / 3} \frac{A_{\text {side }}}{A_{\text {inner }}}+1 .
\end{gathered}
$$

Each single-phase case is first run with turbulent mixing disabled, so as to see that CTF predicts the correct single-phase flow distribution. Results show that CTF does predict the correct flow distribution; however, not within the axial length of the test section, which is $1 \mathrm{~m}$. The CTF model is extended to $7 \mathrm{~m}$ to show that the correct flow split is eventually achieved. Figures 15 and 16 show the results of running CTF with no turbulent mixing for Case 1 and 2 of the $2 \times 2$ facility, respectively.

The channel mass flux results are normalized as shown in Equation 13. The figures show four important pieces of information:

1. The CTF normalized channel mass fluxes are shown for corner, side, and inner type channels (red, blue, and green) using the solid lines,

2. The analytical solution for the flow split (obtained using Equations 30, 31, and 32) is shown with the three horizontal dashed lines using the same color scheme to denote channel types,

3. The experimental measurements are shown with the dot-dash lines using the same color scheme for denoting channel type, and

4. The shaded regions show the maximum experimental measurement uncertainty for channel mass flux $(5 \%)$, as quoted in the $2 \times 2$ technical report [3].

The figures shows that the trend for flow to migrate into the lower resistance inner channel and out of the higher resistance corner channel is correctly predicted. The CTF channel flows hit the expected values at about $6 \mathrm{~m}$. The experimental results are not exactly the same as the theoretical values since turbulent mixing drives momentum from the higher velocity inner channel back to the corner and side channels. However, note that the experimental results are obtained within $1 \mathrm{~m}$ of test section length.

Figures 17 and 18 show the same results with turbulent mixing enabled in CTF and the singlephase turbulent mixing coefficient, $\beta$, set to 0.007 (the ideal value discovered in Section 2.2).

Enabling turbulent mixing leads to a new mechanical equilibrium point in CTF. The inner channel flow does not go as high and corner channel flow does not go as low. The results seem to indicate that the CTF mixing coefficient may be slightly too high for this experimental facility. The 


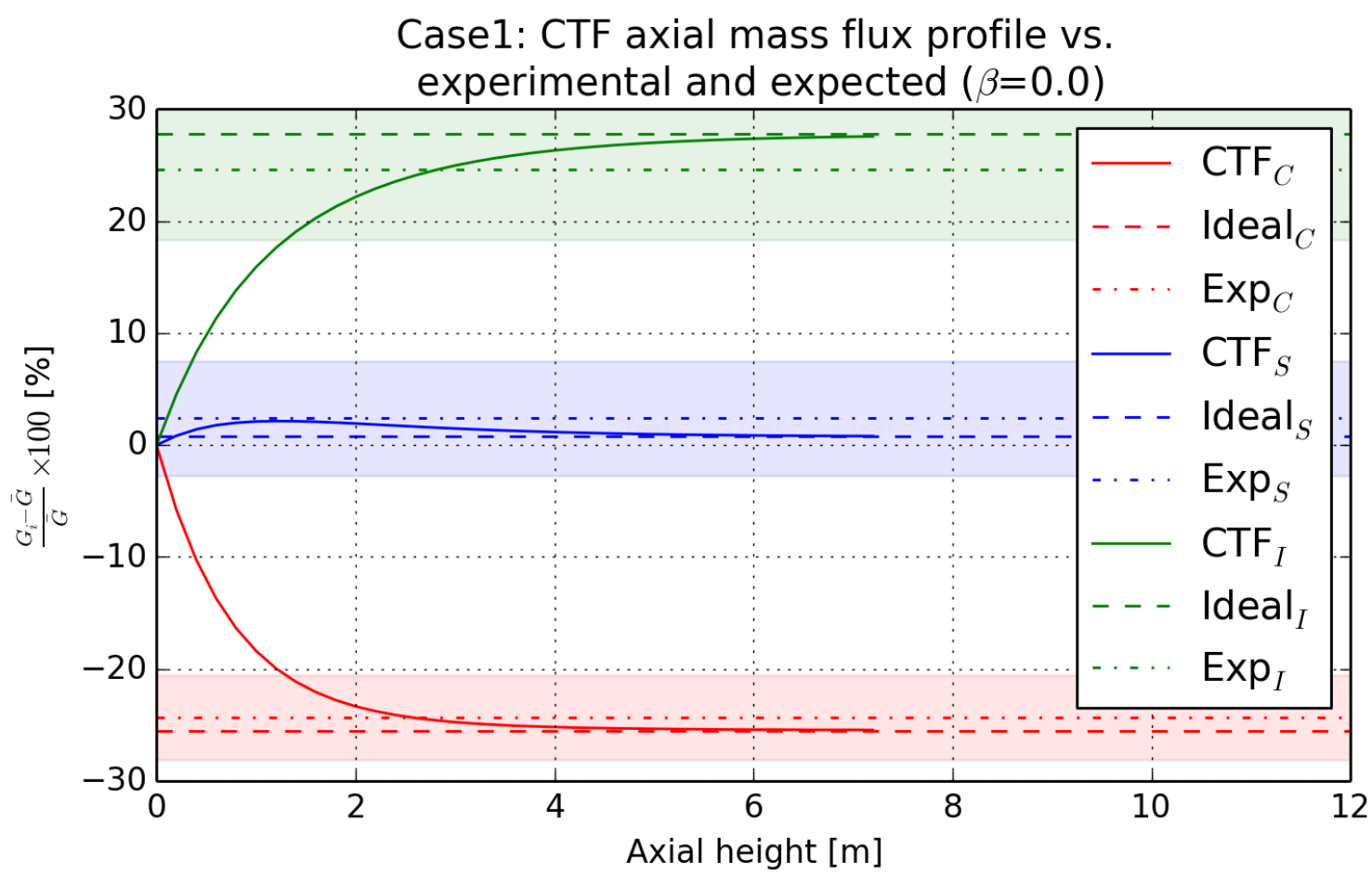

Figure 15. CTF-predicted flow split (no mixing) for $2 \times 2$ Case 1 single-phase test compared with experimental values and ideal flow split.

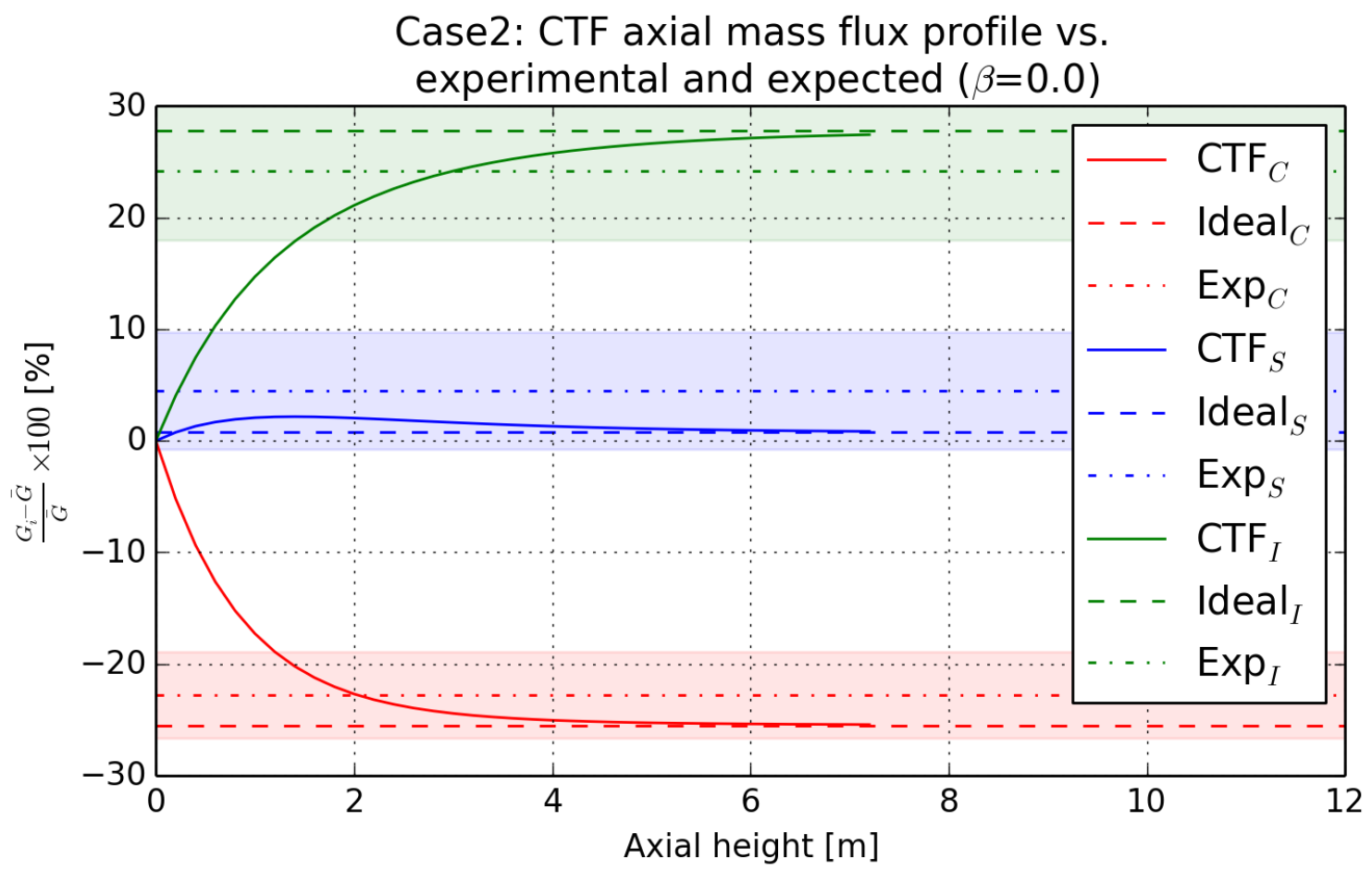

Figure 16. CTF-predicted flow split (no mixing) for $2 \times 2$ Case 2 single-phase test compared with experimental values and ideal flow split. 


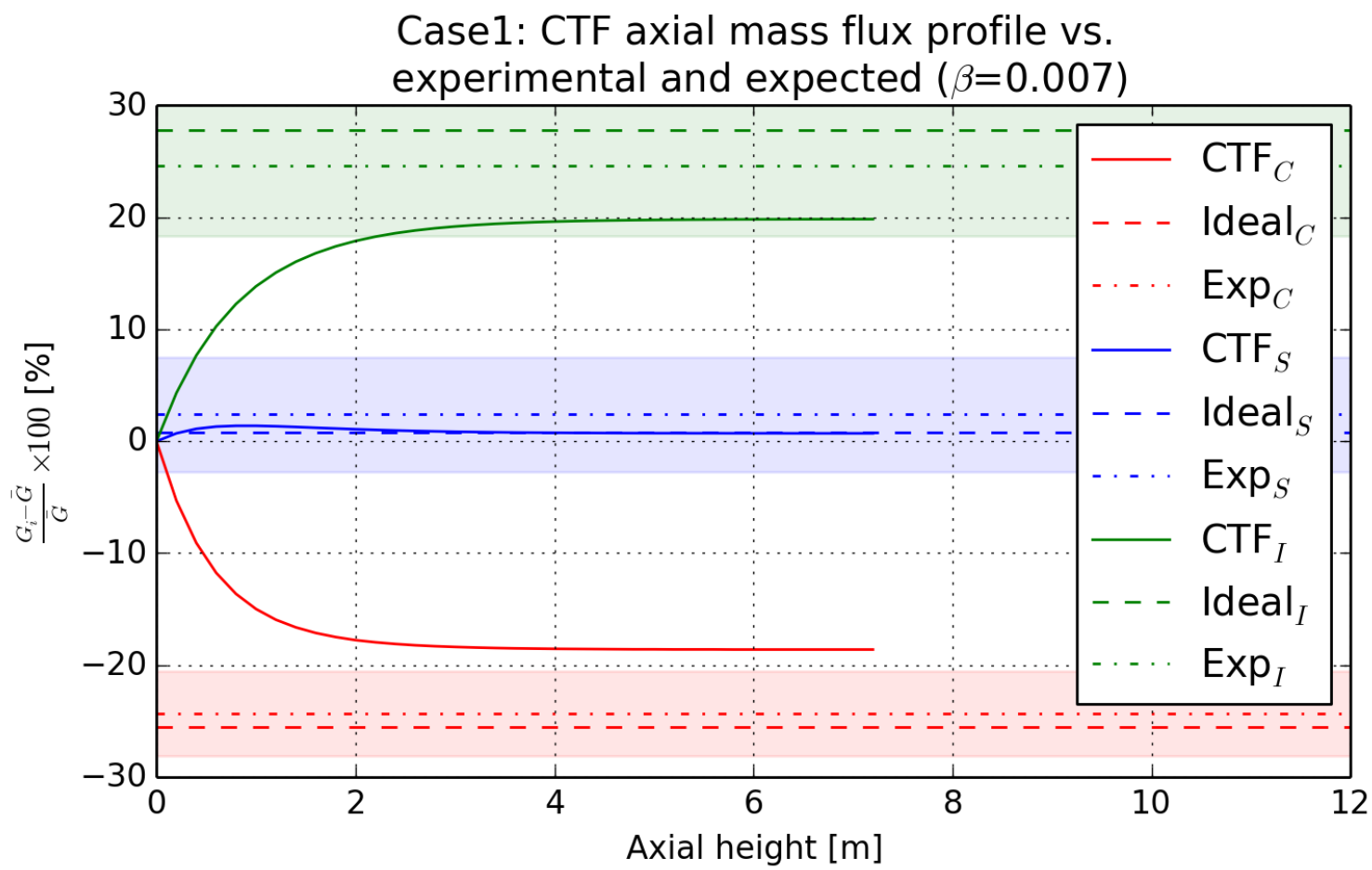

Figure 17. CTF-predicted flow split (with $\beta$ set to 0.007 ) for $2 \times 2$ Case 1 single-phase test compared with experimental values and ideal flow split.

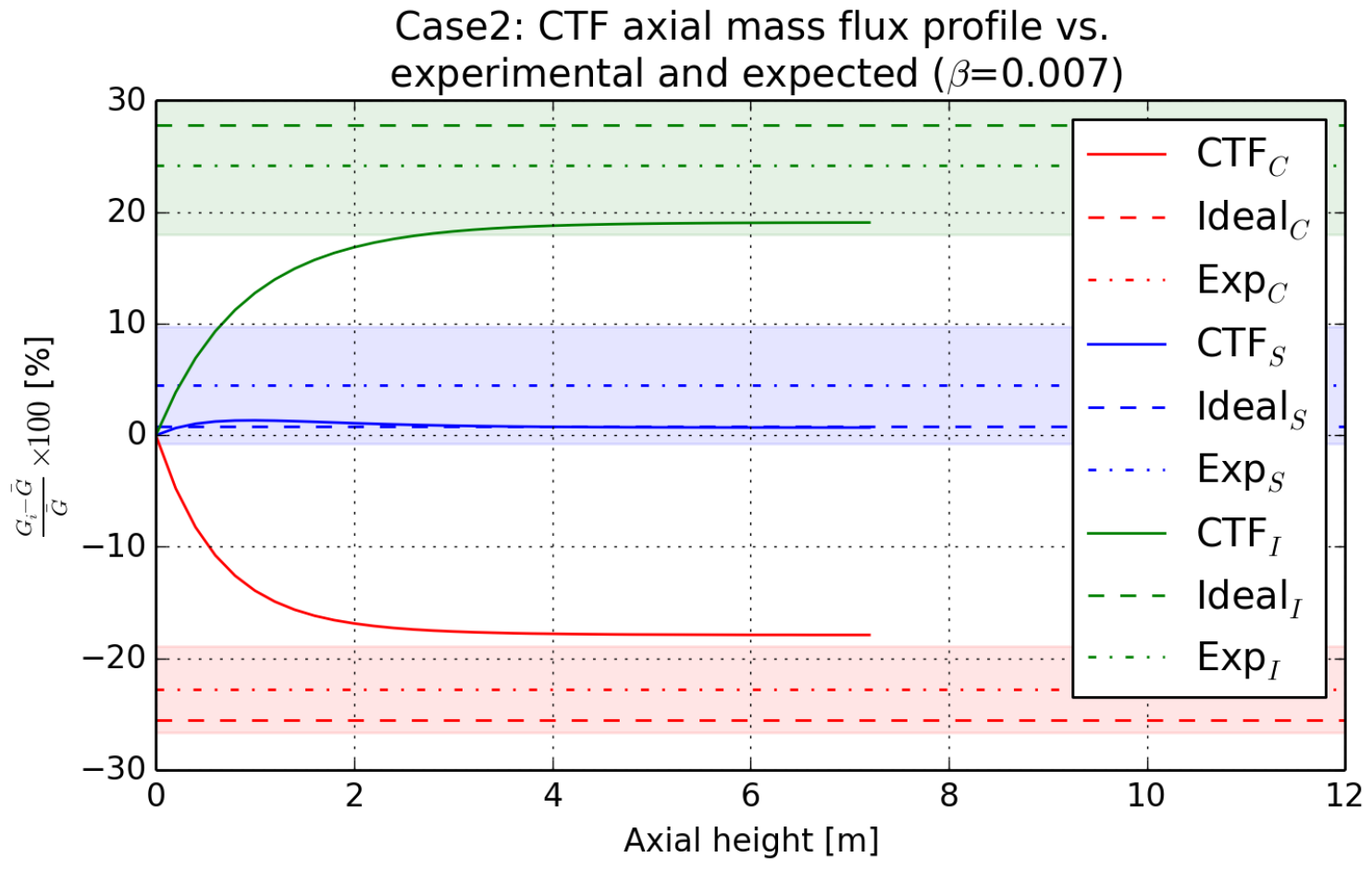

Figure 18. CTF-predicted flow split (with $\beta$ set to 0.007 ) for $2 \times 2$ Case 2 single-phase test compared with experimental values and ideal flow split. 
CTF inner channel mass flux is lower than the experimental inner channel measurement and the CTF corner channel mass flux is higher the than the experimental corner measurement. The corner channel prediction is just outside of the measurement uncertainty bands.

Adjusting the mixing coefficient down to $\beta=0.0035$ leads to a more favorable prediction of the flow split, as shown in Figures 19 and 20.

Similar to the cases without mixing, the flow takes about 5-6 $\mathrm{m}$ to reach equilibrium distribution in the CTF model. Since Case 2 has a mass flux that is twice that of Case 1, it appears that the magnitude of flow has no significant effect on the distance to reach equilibrium flow distribution. 


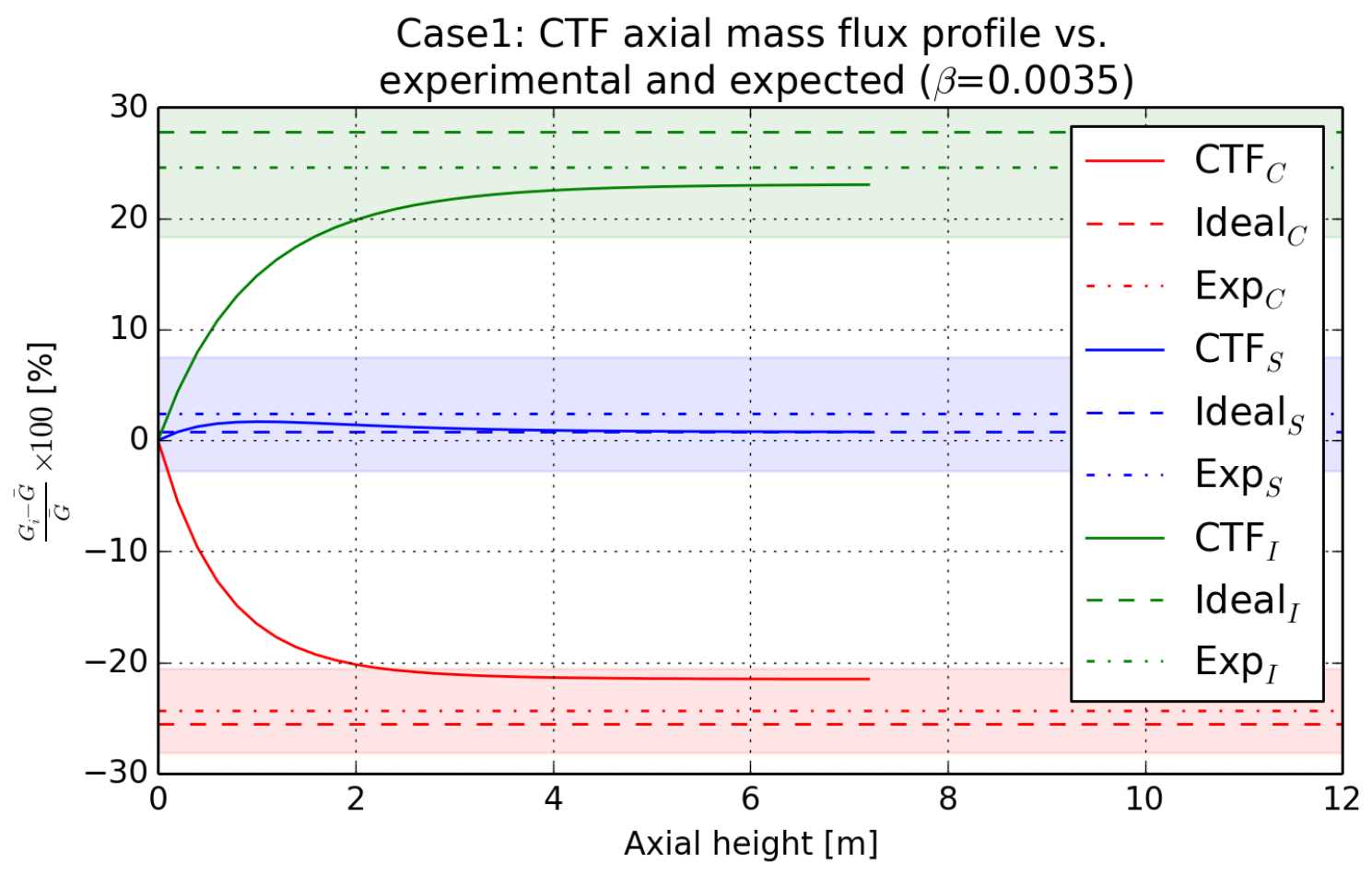

Figure 19. CTF-predicted flow split (with $\beta=0.0035$ ) for $2 \times 2$ Case 1 single-phase test compared with experimental values and ideal flow split.

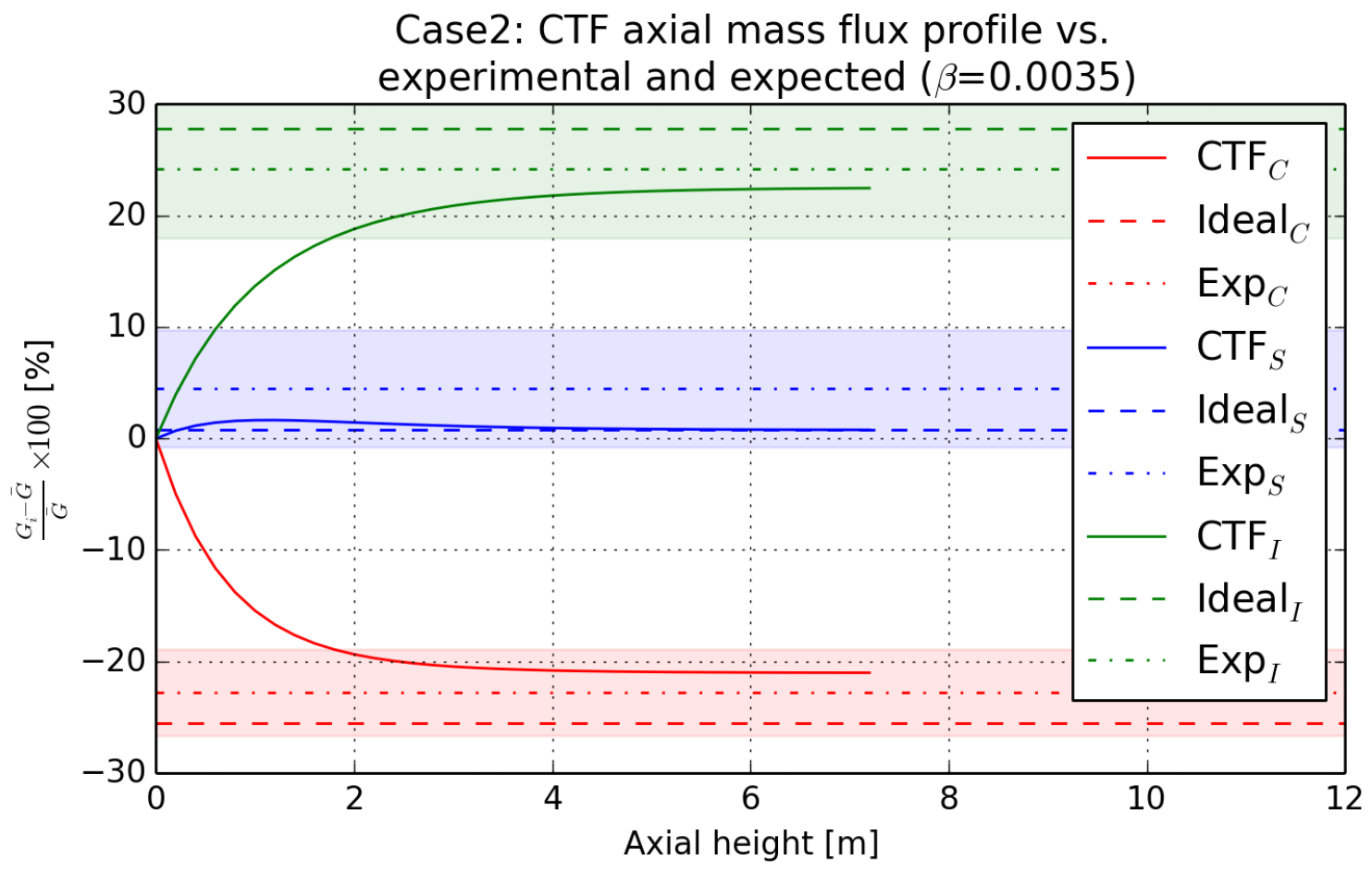

Figure 20. CTF-predicted flow split (with $\beta=0.0035$ ) for $2 \times 2$ Case 2 single-phase test compared with experimental values and ideal flow split. 


\subsection{GE $3 \times 3$ Validation}

Facility Description The GE $3 \times 3$ facility [11] is a classic test for assessing inter-subchannel mixing because mass flux and quality measurements are made for individual subchannel types. A $3 \times 3$ electrically heated bundle with BWR geometry is used. The axial and radial power profiles are uniform for all of these test cases. The same bundle is used for all the tests. Bundle power, flow rate, and inlet subcooling are varied between different experimental cases. Measurements are made at the outlet of the facility using an isokinetic sampling approach, similar to the approach used in the $2 \times 2$ facility. The estimated maximum error in subchannel flow measurements is $\pm 2 \%$. The authors determined that the error in the exit equilibrium quality measurements were \pm 0.02 . Additional details on the facility can be found in the experiment technical report [11] as well as the CTF Validation Manual [4].

Discussion of Results Because this section of the study is reviewing the ability to predict correct single-phase flow distribution, only the four single-phase cases (1B, 1C, 1D, and 1E) are run. These cases are not currently included in the CTF Validation Manual [4]. Considering the difficulty CTF exhibited in correctly predicting the flow split for the $2 \times 2$ facility, it is prudent to assess the flowdistribution prediction for the GE $3 \times 3$ case before analyzing the two-phase void drift cases.

The cases are first run without spacer grids and with turbulent mixing disabled. The ideal flow split is calculated using the same approach as Section 2.4. Again, flow results are displayed as normalized values, the ideal flow split is shown with dashed horizontal lines, and the measured results are shown with dot-dash lines. The measurement uncertainty is shown as a colored, shaded region around the measurement line. Figures 21-24 show the result of these predictions for the four single-phase cases with no turbulent mixing or form losses.

Similar to the $2 \times 2$ facility tests, CTF predicts the correct flow rate distribution; however, it is interesting to note that the equilibrium distribution is achieved in a much shorter length of the facility. The flow distribution is nearly in equilibrium at the $1.8 \mathrm{~m}$ location, which is the exit of the facility; whereas, the $2 \times 2$ facility took nearly $5 \mathrm{~m}$ to reach equilibrium.

Adding the grids and turbulent mixing produces the results shown in Figures 25-28. The channel mass fluxes are closer to the bundle-average value as a result of the turbulent mixing model being enabled. The addition of the grids results in a "choppy" appearance of the axial mass flux distributions. Because the grid form loss coefficient is the same for all channels, the grids have an "equalizing" effect, pushing inner channel flow back into the side and corner channels. The redistribution is driven by the fact that the higher-velocity inner channel flow experiences a greater pressure loss than the lower-velocity corner channel at the grid location. The imbalance in flow losses causes flow to migrate from the higher resistance inner channel to the lower resistance corner channel. The redistribution lasts for a short period downstream of the grid loss and soon reverses so that flow moves back into the inner channel.

All single-phase predicted outlet mass fluxes are compared with their measured counterparts in Figure 29. Each color represents a unique channel type: red for corner, blue for side, and green for inner. Inner- and side-predicted mas fluxes match experimental values closely, having relative rootmean-square of error (rRMSE) (Equation 34) values that are close to experimental measurement uncertainty. Corner results vary from experimental values by a much larger degree.

$$
\begin{gathered}
\mathrm{rRMSE}=\sqrt{\frac{1}{N} \sum_{i=1}^{N} E_{\mathrm{rel}, i}^{2}}, \text { where } \\
E_{\mathrm{rel}}=\frac{x_{\text {measured }}-x_{\text {predicted }}}{x_{\text {measured }}}
\end{gathered}
$$

The excellent agreement between CTF and the experiment found in this single-phase study adds more credibility to the two-phase void drift study performed with this data in the CTF Validation Manual [4]. However, it is noted that the turbulent mixing model in this study is different from the one used in the Validation Manual. The original GE $3 \times 3$ study was done using the Rogers \& Rosehart correlation to calculate the mixing coefficient. This choice is further investigated in this section and the two-phase void drift modeling section. 


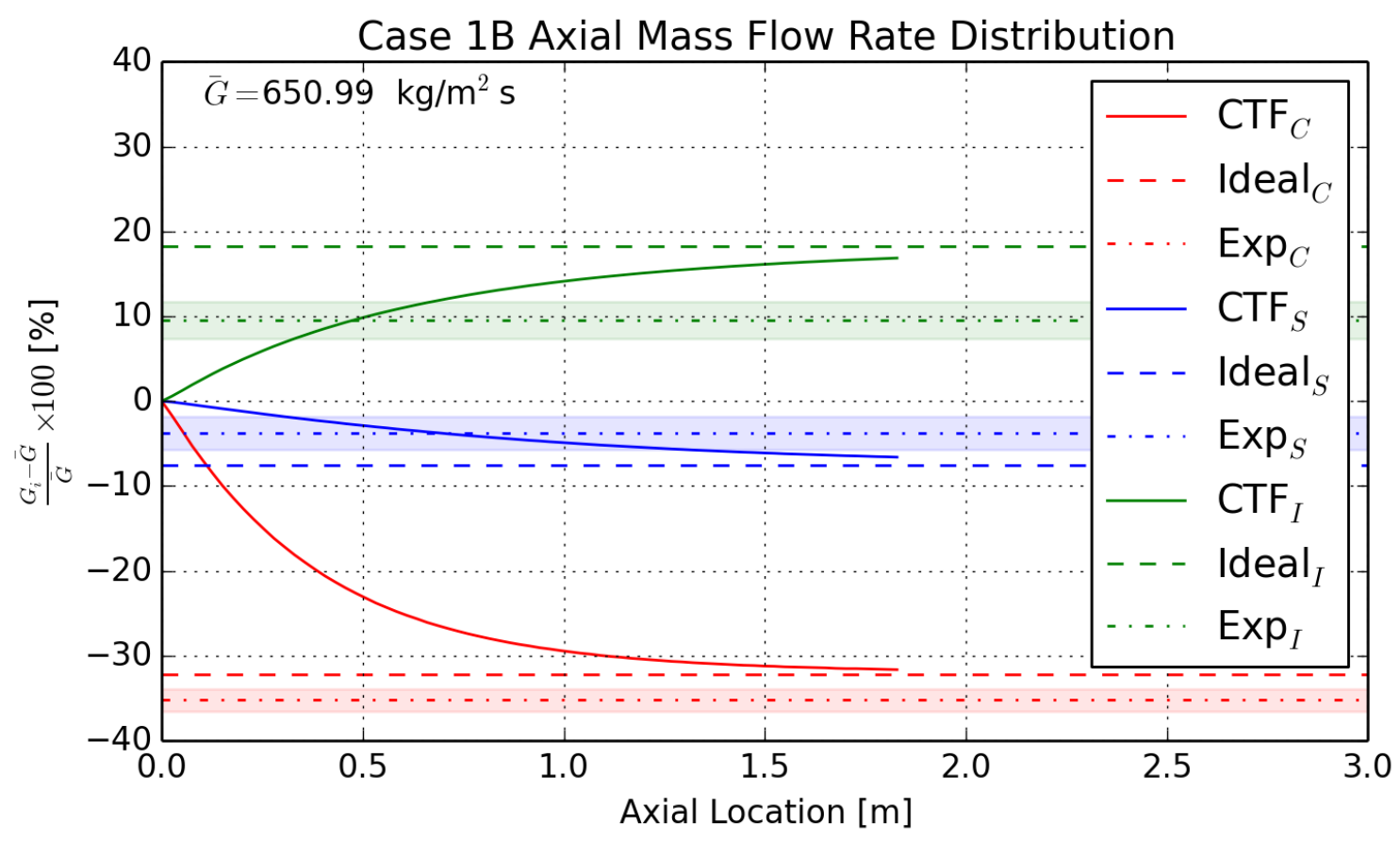

Figure 21. CTF-predicted flow split compared with ideal and measured results for GE $3 \times 3$ Case 1B (no mixing or spacer grids in model).

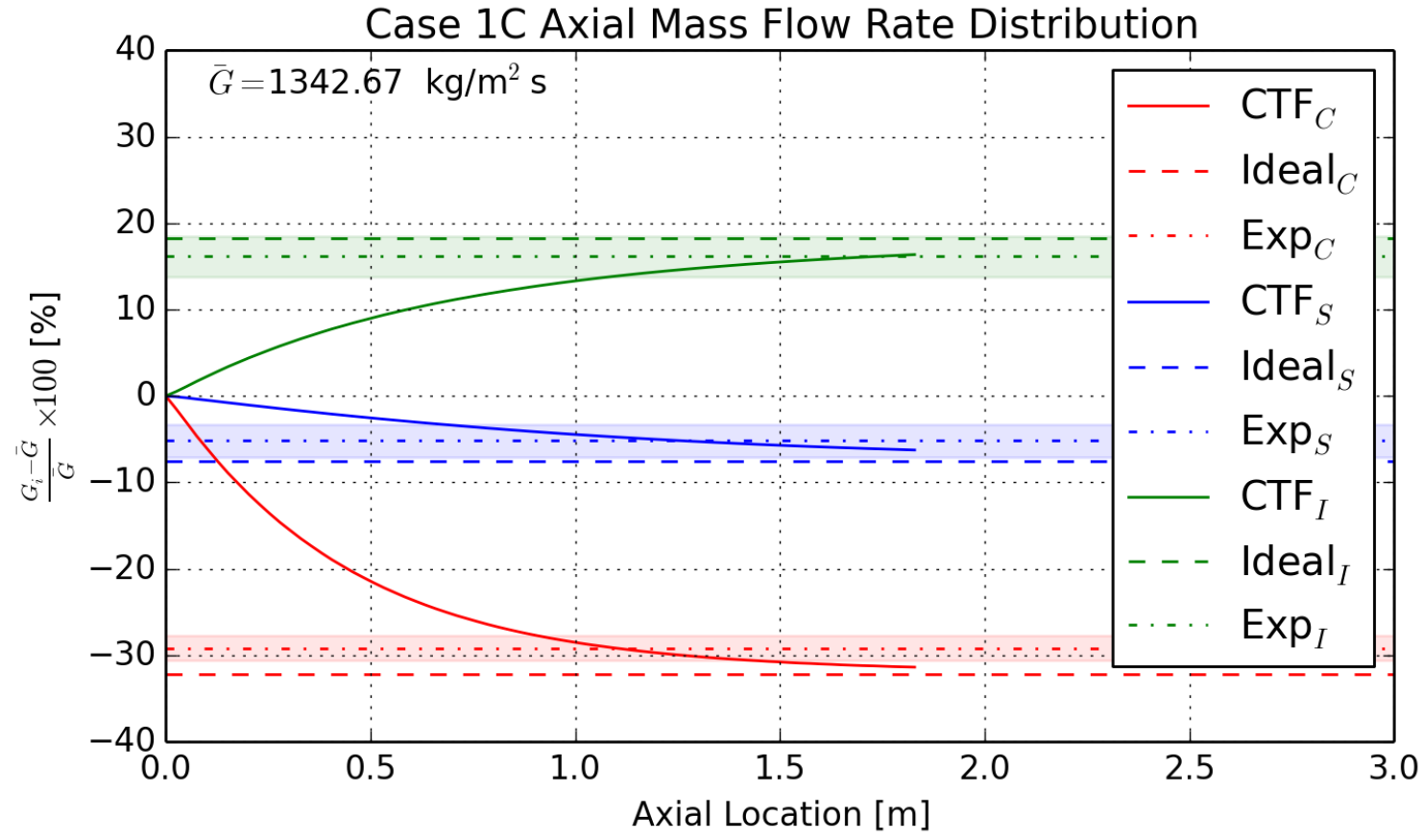

Figure 22. CTF-predicted flow split compared with ideal and measured results for GE $3 \times 3$ Case 1C (no mixing or spacer grids in model). 


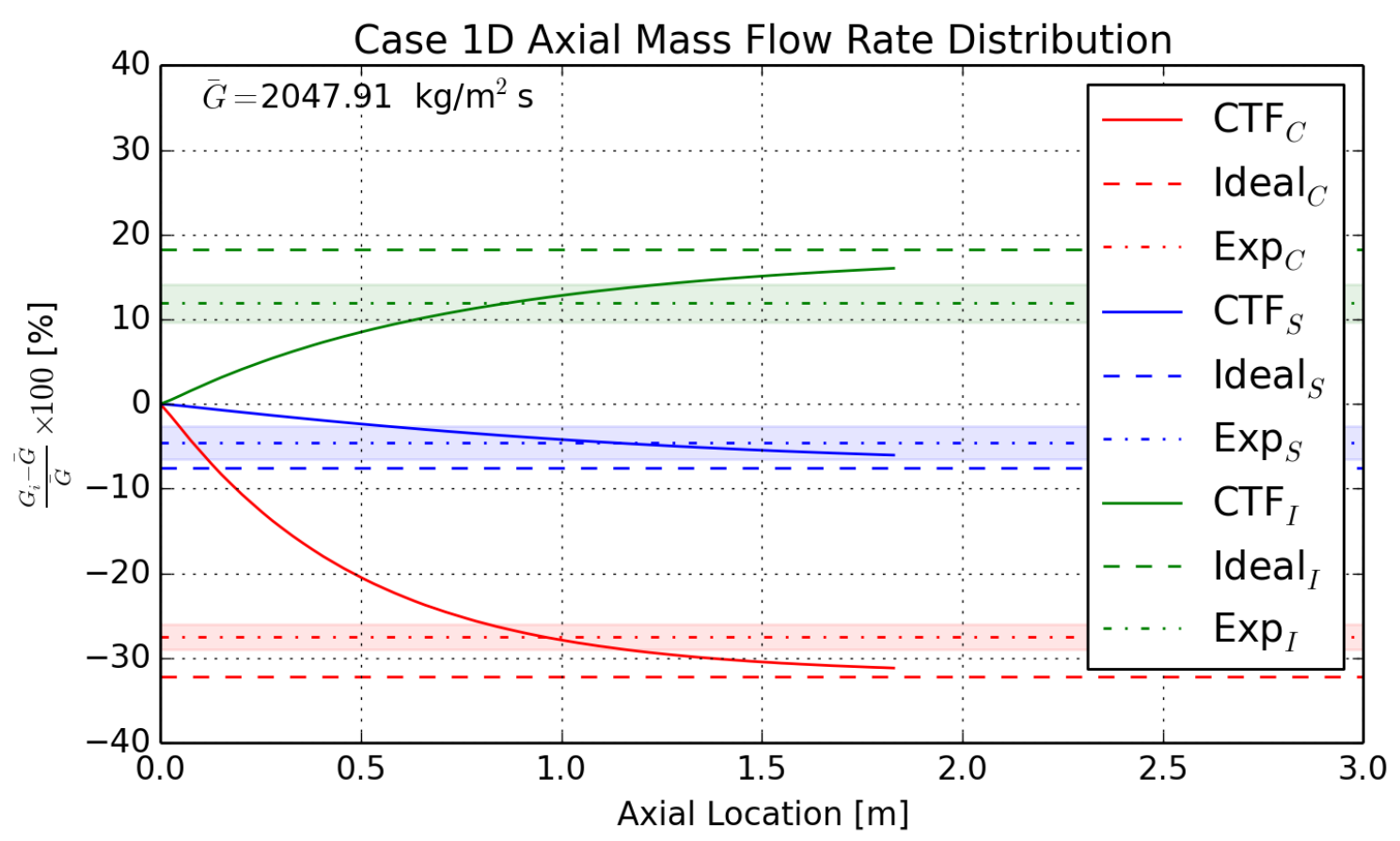

Figure 23. CTF-predicted flow split compared with ideal and measured results for GE $3 \times 3$ Case 1D (no mixing or spacer grids in model).

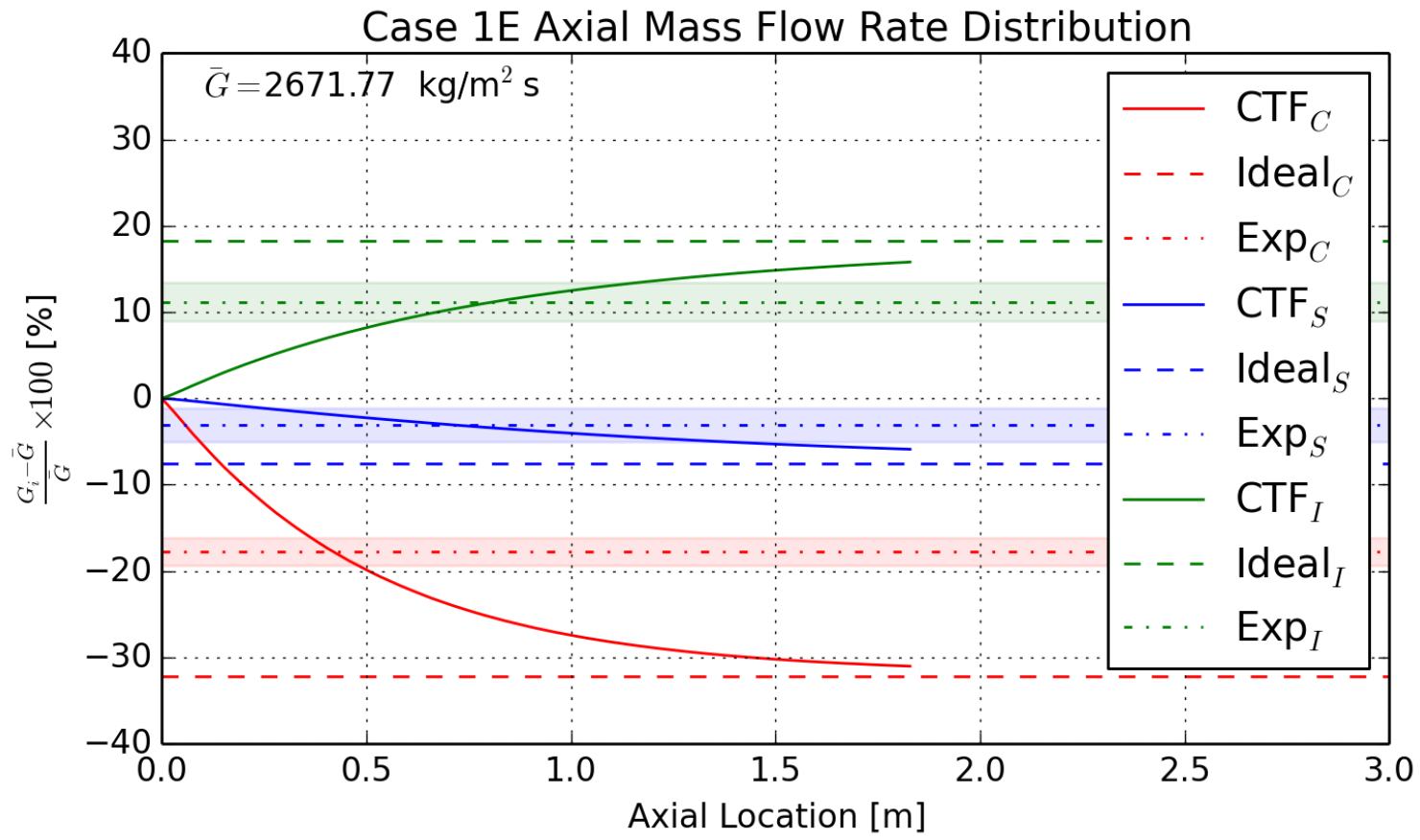

Figure 24. CTF-predicted flow split compared with ideal and measured results for GE $3 \times 3$ Case 1E (no mixing or spacer grids in model). 


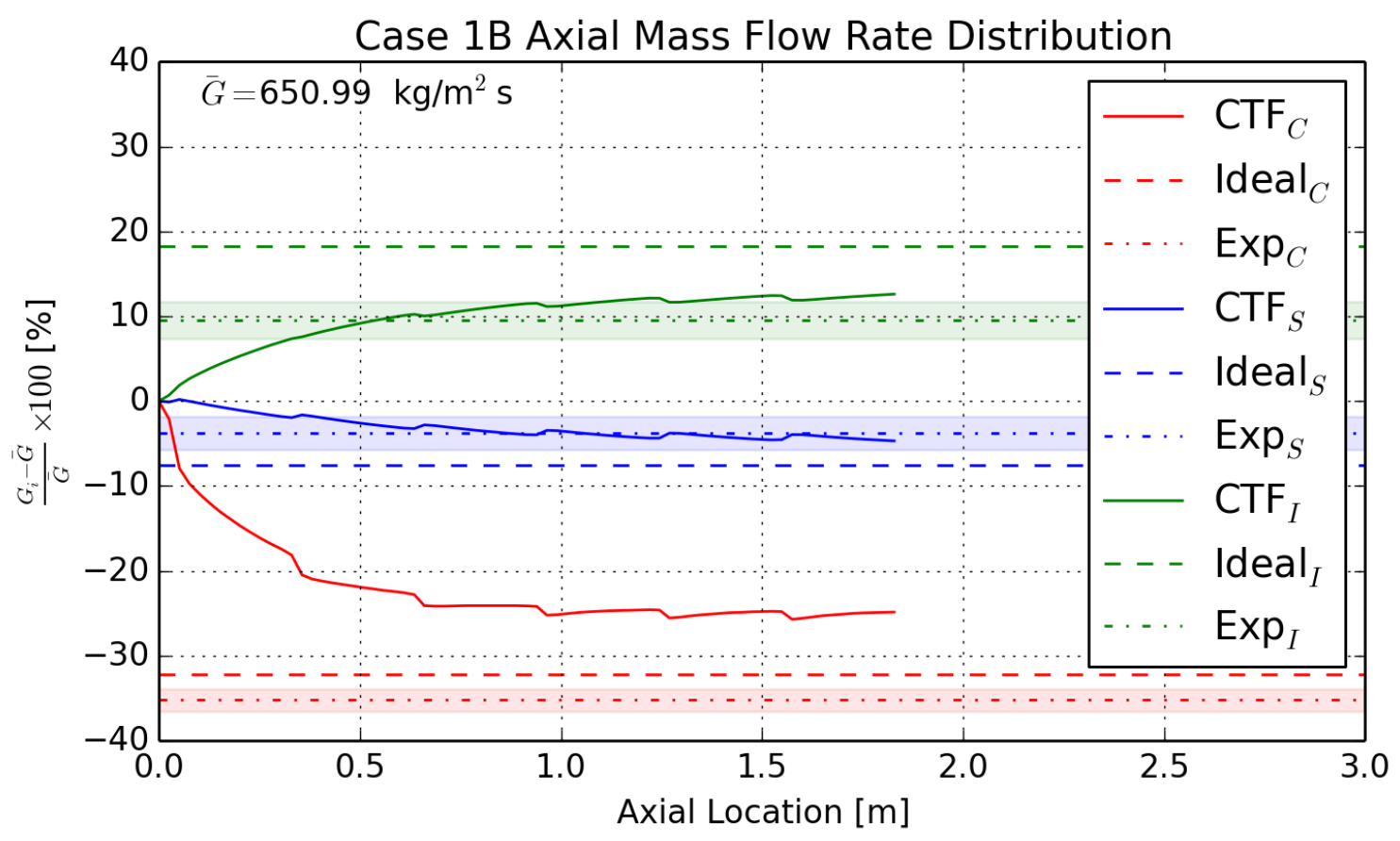

Figure 25. CTF-predicted flow split compared with ideal and measured results for GE $3 \times 3$ Case 1B (with $\beta=0.007$ ).

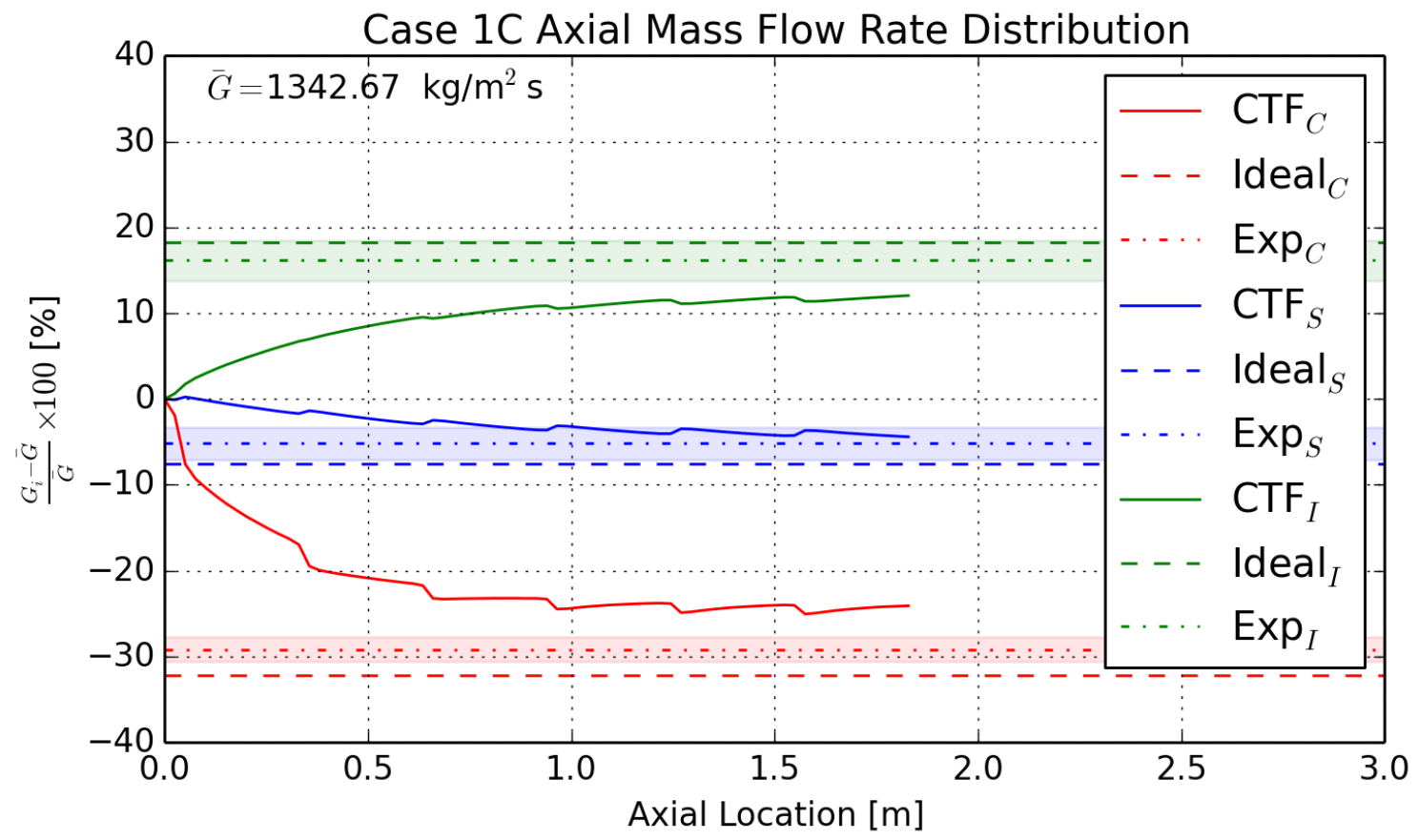

Figure 26. CTF-predicted flow split compared with ideal and measured results for GE $3 \times 3$ Case 1C (with $\beta=0.007$ ). 


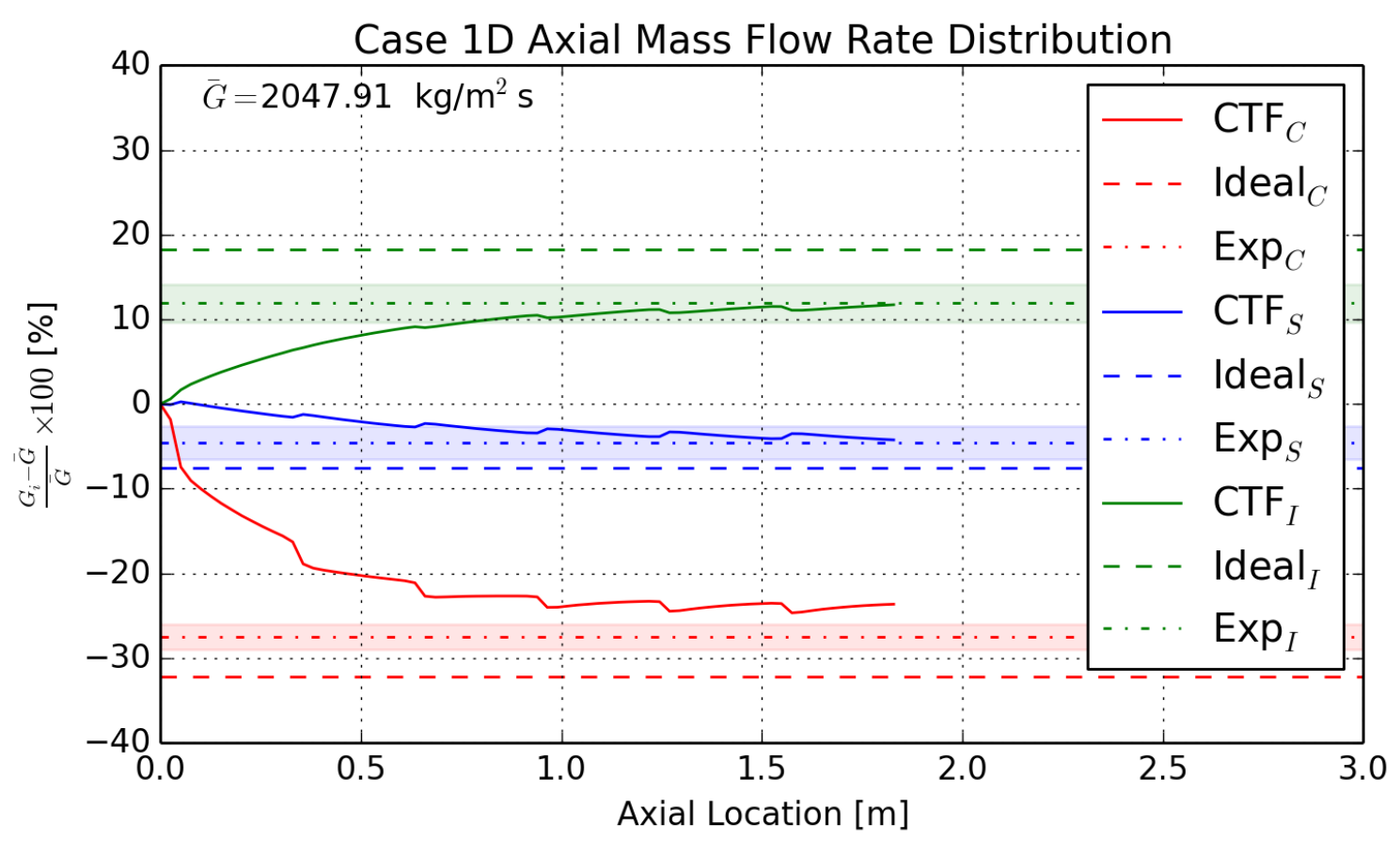

Figure 27. CTF-predicted flow split compared with ideal and measured results for GE $3 \times 3$ Case 1D (with $\beta=0.007$ ).

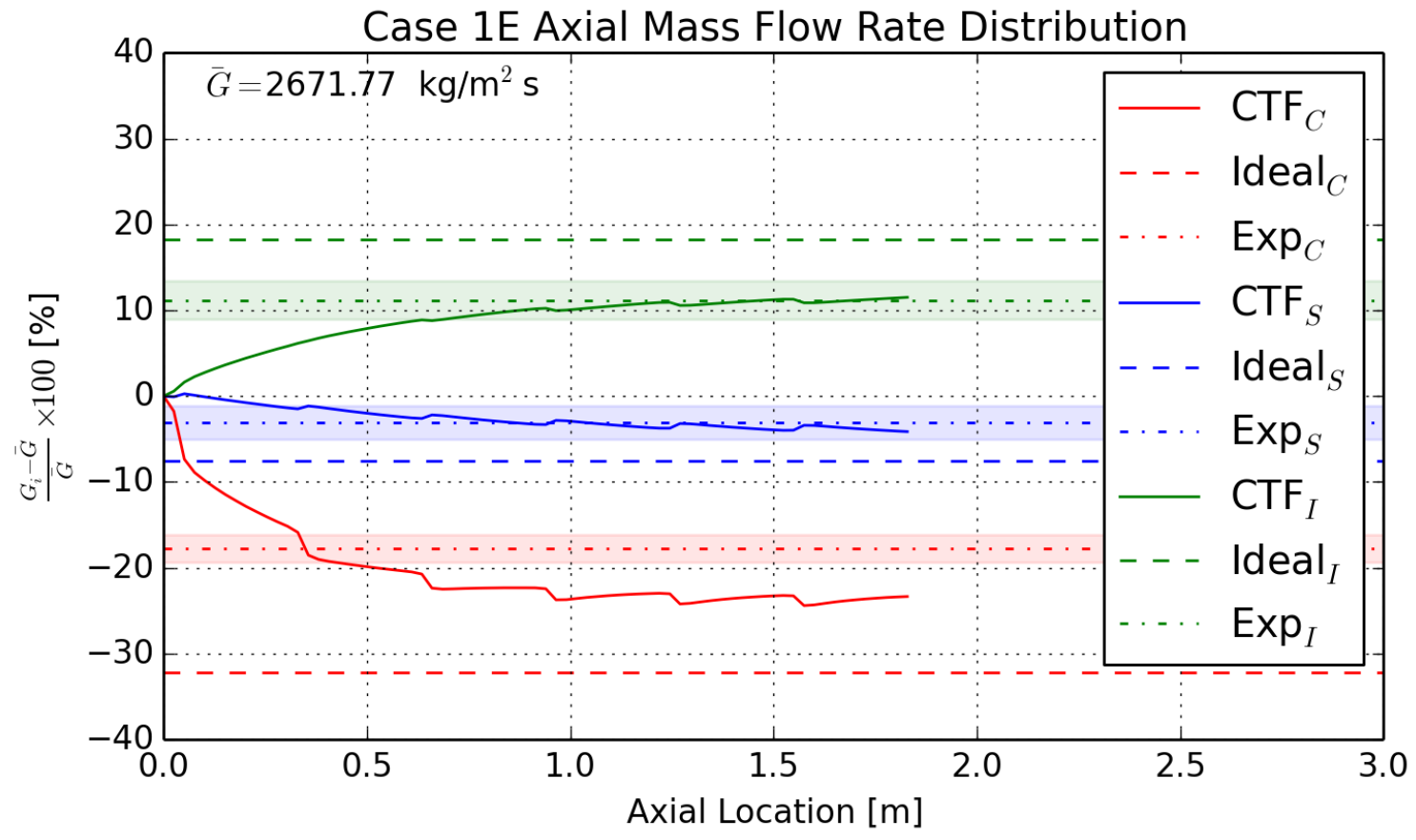

Figure 28. CTF-predicted flow split compared with ideal and measured results for GE $3 \times 3$ Case $1 \mathrm{E}$ (with $\beta=0.007$ ). 
It has been shown in Section 2.2 that the Rogers \& Rosehart model tends to exaggerate the mixing in the system. The single-phase cases are re-run using the Rogers \& Rosehart model for $\beta$ and measured versus predicted exit mass fluxes are shown in Figure 30. Side and inner results are largely unaffected, but the corner channel experiences a more significant drop in accuracy. Overall rRMSE increases by two percentage points. The average error (Equation 36) increases from 3.8\% to $4.8 \%$.

$$
\bar{E}_{\text {rel }}=\Sigma_{i=1}^{N} \frac{\left|x_{\text {measured }, i}-x_{\text {predicted }, i}\right|}{x_{\text {measured }, i}} \times 100
$$




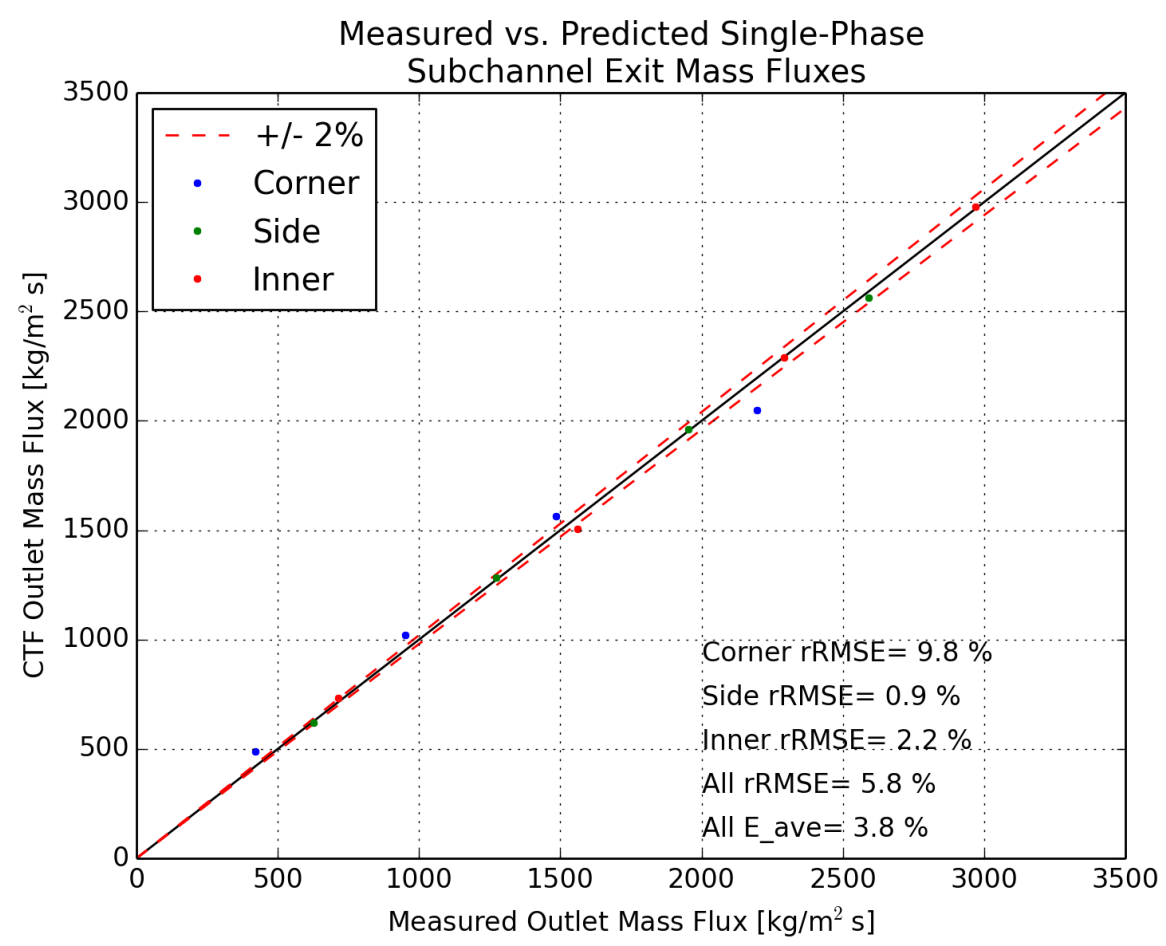

Figure 29. Comparison of predicted and measured exit mass flux for GE $3 \times 3$ singlephase cases using $\beta=0.007$.

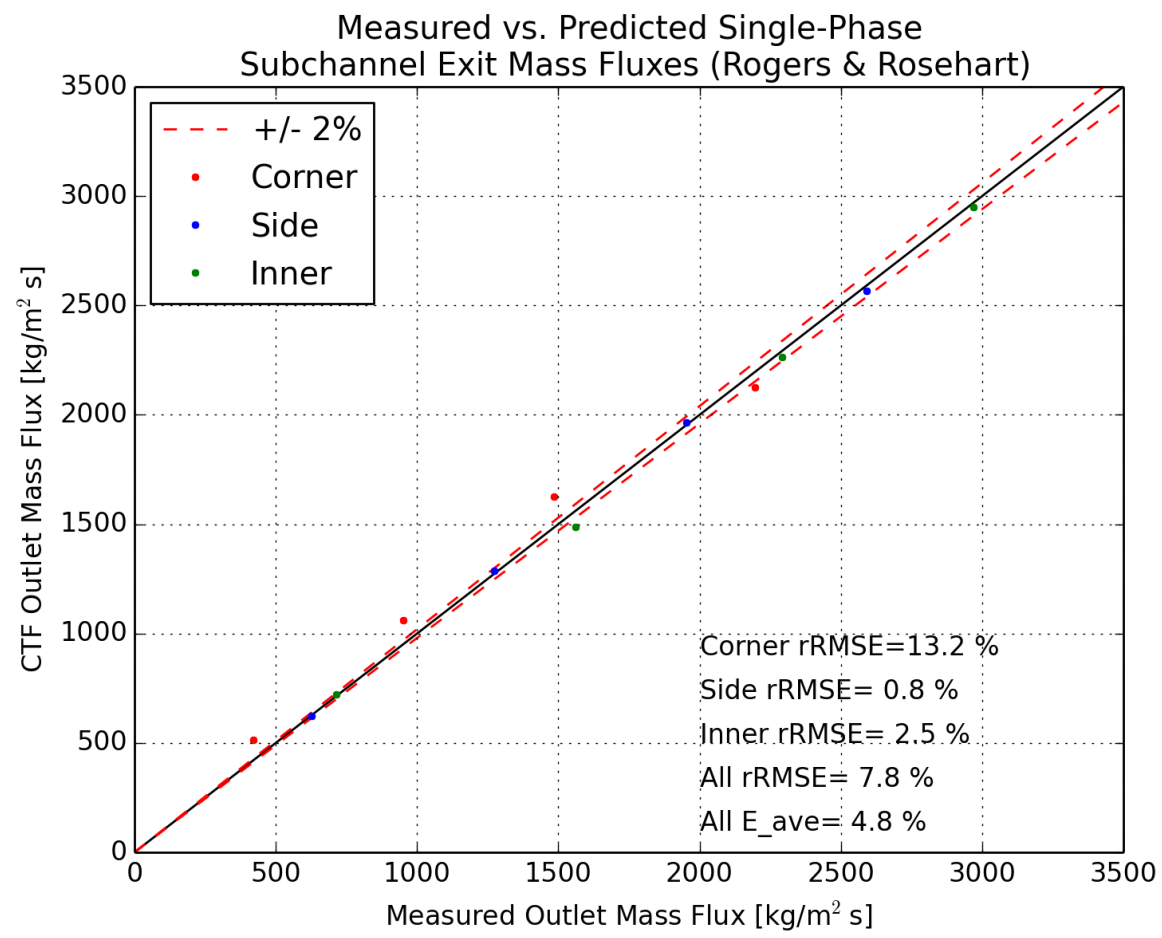

Figure 30. Comparison of predicted and measured exit mass flux for GE $3 \times 3$ singlephase cases using Rogers \& Rosehart to calculate $\beta$. 


\section{VOID DRIFT VALIDATION}

\section{$3.1 \quad$ RPI $2 \times 2$ Validation}

The $2 \times 2$ facility has been introduced in Section 2.4. The single-phase tests performed in the facility were modeled in that section. The facility also includes a series of two-phase tests with inlet void ranging from 20 to $50 \%$. Several attempts are made to model the two phase tests of this facility; however, limited success is achieved when attempting to run the models at the atmospheric pressure specified in the test specification.

It is not possible to model the facility using the non-condensable gas model in CTF because $\mathrm{CTF}$ assumes that the non-condensable gas mixture fills the vapor phase. At atmospheric pressure and room temperature, any vapor in the model condenses away, forcing the non-condensable gas void fraction to zero. Therefore, it is necessary to model the facility as a two-phase, steam/water mixture. It is possible to shut off the evaporation/condensation term in CTF so that the vapor phase behaves as a non-condensable gas.

The first attempt at modeling the facility involves using the experimental pressure as the outlet boundary condition in CTF. Running a two-phase mixture at this pressure leads to large gradients in the steam and liquid fluid properties, which makes it more difficult for the code to converge on a steady-state solution. An example of the code convergence terms is shown in Figure 31. As indicated in the figure, all convergence terms simply oscillate for the duration of the simulation.

It is important to look at actual simulation results behavior throughout the simulation. The exit vapor void is checked in the nine channels of the model at each iteration. They are plotted in Figure 32. The void almost settles on a constant value in each of the channels. The corner channel void (Channels 1, 3, and 9) seem to be experiencing a slow oscillation. However, note that there is no symmetry in the results. The corner channel void (Channels 1, 3, 7, and 9) should all be identical in the converged solution. Likewise, the side channel void (Channels 2, 4, 6, and 8) should be identical.

The case is rerun with the gaps disabled (a collection of nine isolated subchannels) as a sanity check. Results are shown in Figure 33. Here, we see a smooth change in void over the simulation. The simulation only takes about 4,000 iteration to converge. All side-channel voids are identical, and all corner-channel voids are identical, as expected.

It is found that increasing pressure in the system is an effective means of improving code convergence. However, large pressure increases are required to allow all cases to converge. The test case pressure is first increased to 50 bar; the inlet enthalpy is increased to keep the mixture two-phase. Figure 34 shows the result. The void distribution is chaotic in time and space. However, observe that increasing the mixture enthalpy, and thus the bundle void, allows the code to converge (Figure $35)$.

Increasing the pressure further to 100 bar allows the code to converge for a wider range of mixture enthalpy. Figure 36 shows a low void case, Figure 37 shows a higher void case, and Figure 38 shows a very high void case. All cases converge nicely. However, the code still has some trouble converging in a certain range of void fractions. Figure 39 demonstrates that the corner channel voids start to diverge slightly at a bundle-average void of around 0.4.

The previous study involving these tests, which is documented in the CTF Validation document [4], used this artificially elevated operating pressure of 100 bar to allow the cases to converge. Running at this much higher pressure increases the vapor-to-liquid density ratio to 0.08 . In reality, this ratio was about 0.002 in the experiments. Furthermore, the inlet Reynolds number increases from roughly 40,000 to about 120,000 because of changing fluid properties. These changes introduce an unknown amount of uncertainty into the results. Furthermore, as noted in Section 2.4, CTF does not predict the equilibrium flow distribution by the outlet of this facility.

It is useful to investigate the effect of lengthening the CTF model. Figure 40 shows the normalized mass flux distribution in the three channel types along the length of the test section. It looks as though the flow distribution at the outlet has almost reached its equilibrium value; however, when the test section is extended slightly in Figure 41, it is seen that the converged flow distribution has not actually reached equilibrium; axial mass fluxes begin to move apart from one another near the end of the bundle, with mass flux increasing in the inside channel and decreasing in the corner 


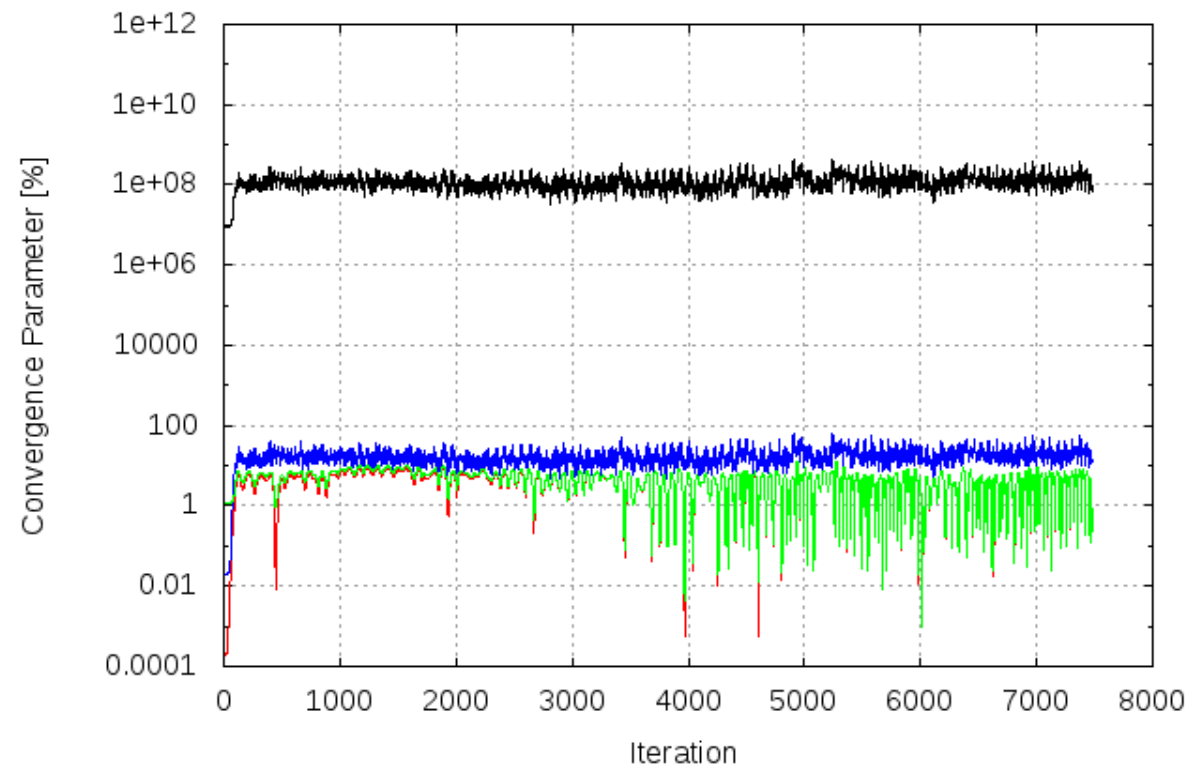

Mass balance

Energy balance

Mass storage Fluid energy storage

Figure 31. CTF convergence terms for Test 3 of the $2 \times 2$ facility when run at atmospheric pressure.

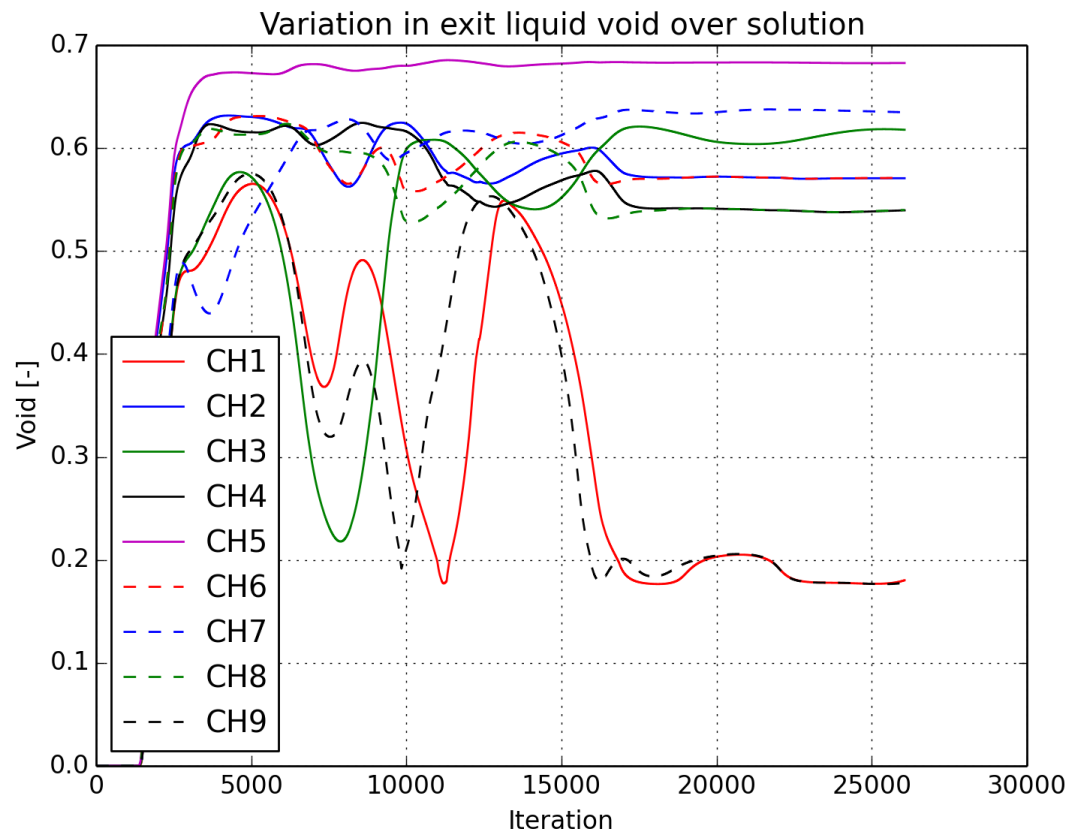

Figure 32. Exit void in Test 3 of $2 \times 2$ facility throughout simulation. 


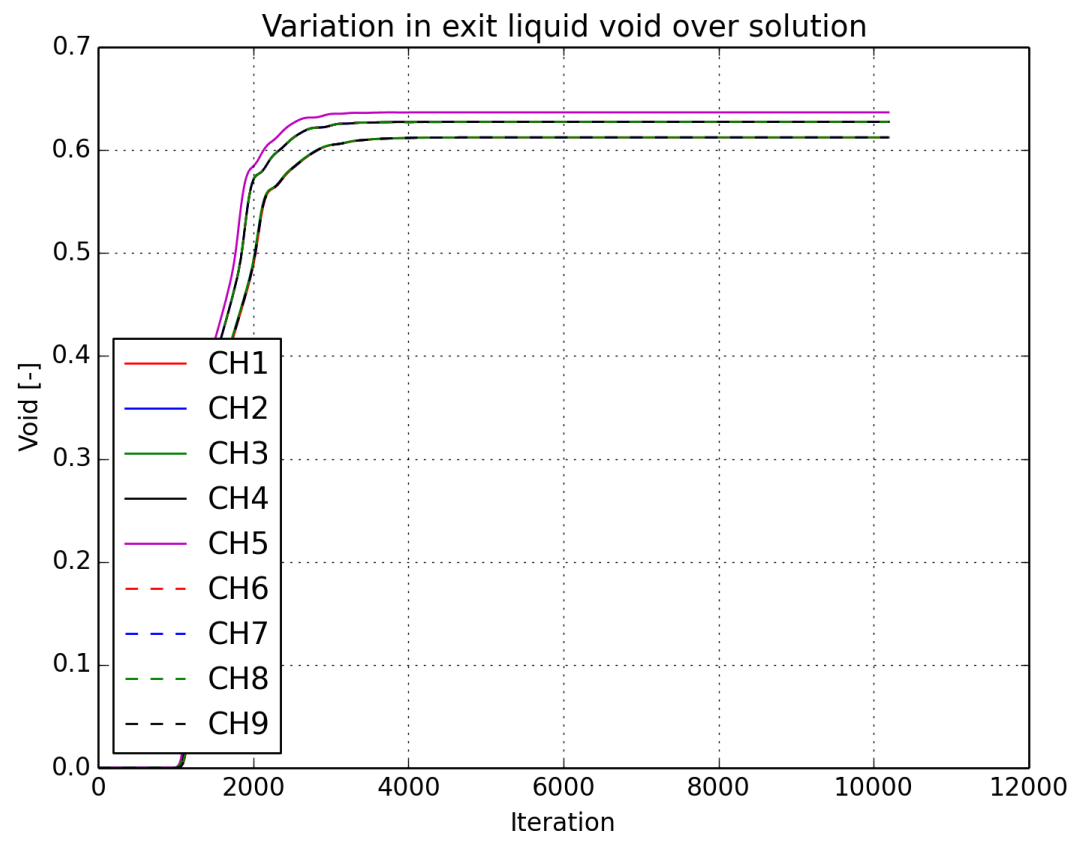

Figure 33. Exit void in Test 3 of $2 \times 2$ facility with gaps disabled.

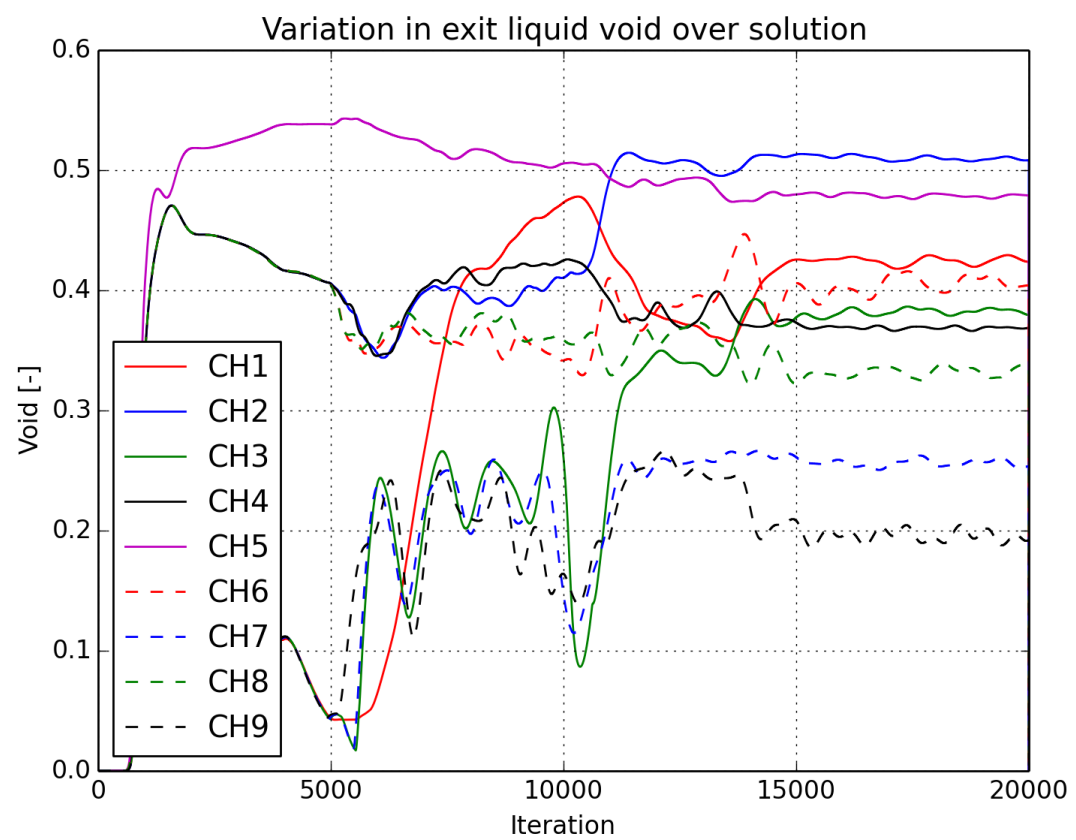

Figure 34. Exit void in Test 3 of $2 \times 2$ with outlet pressure set to 50 bar. 


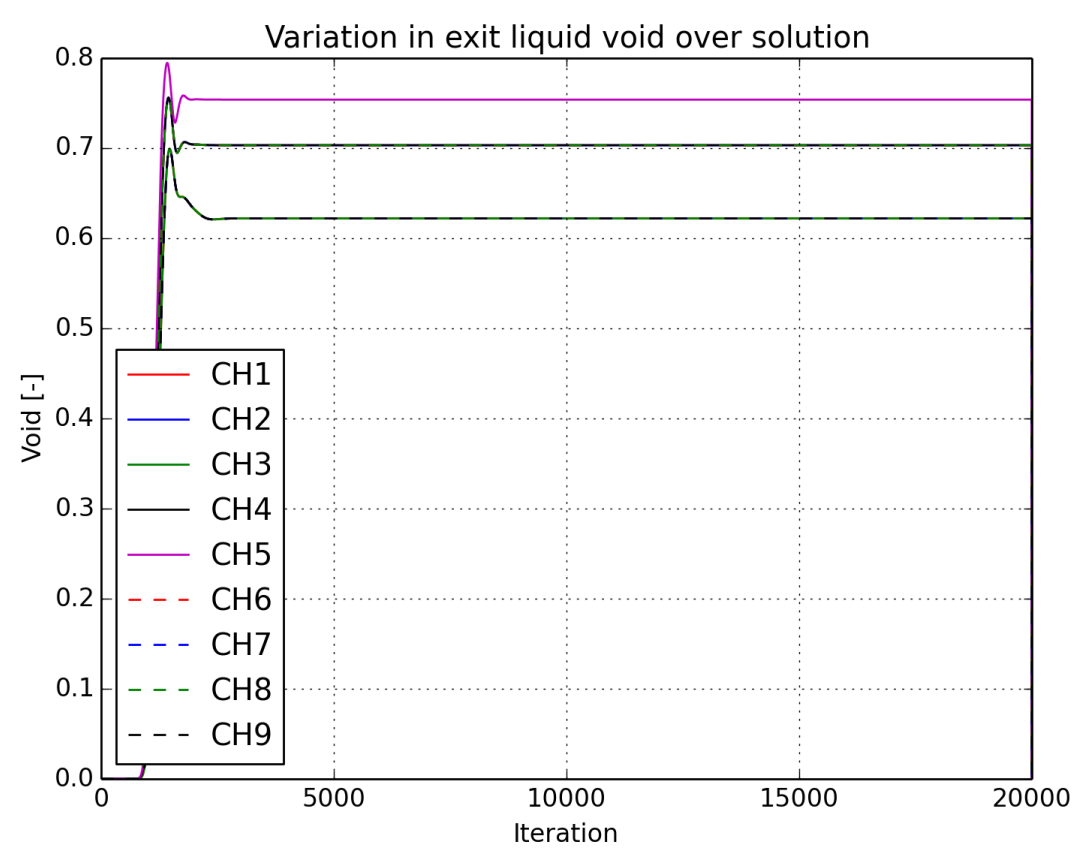

Figure 35. Exit void in Test 3 of $2 \times 2$ with outlet pressure set to 50 bar and mixture enthalpy increased.

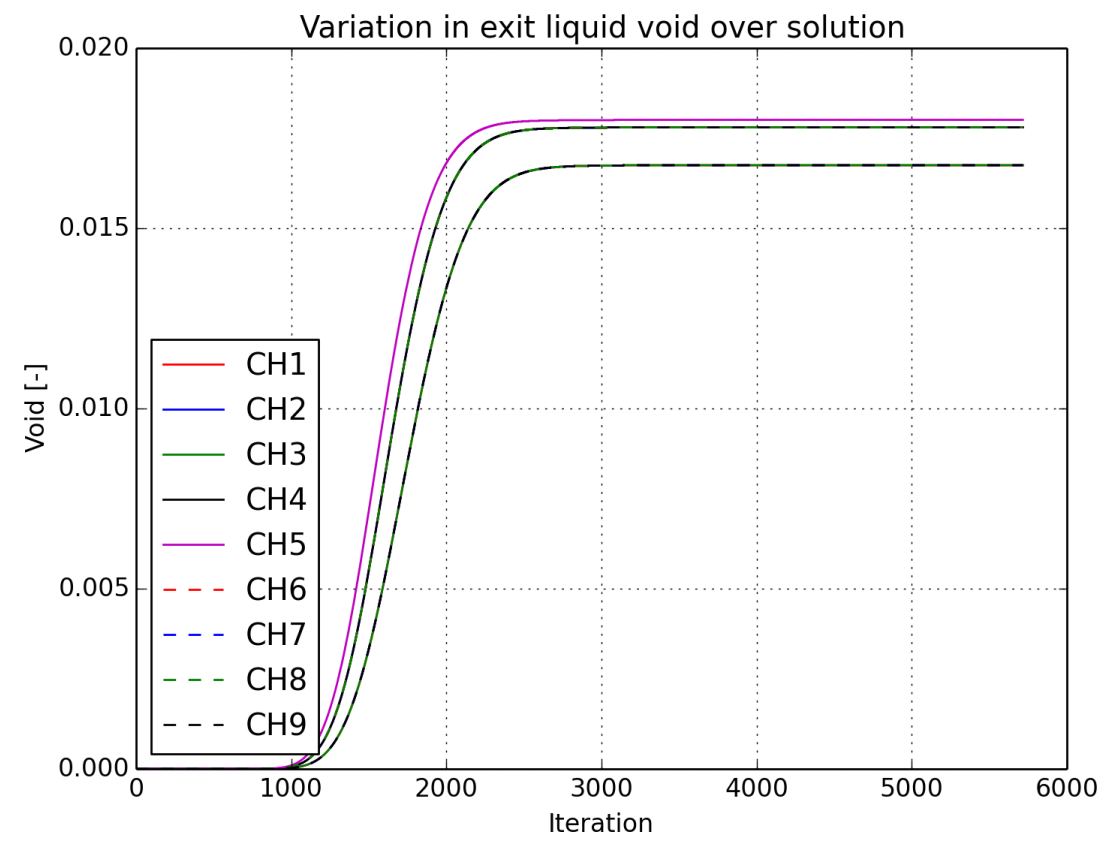

Figure 36. Exit void in Test 3 of $2 \times 2$ with outlet pressure set to 100 bar (low void). 


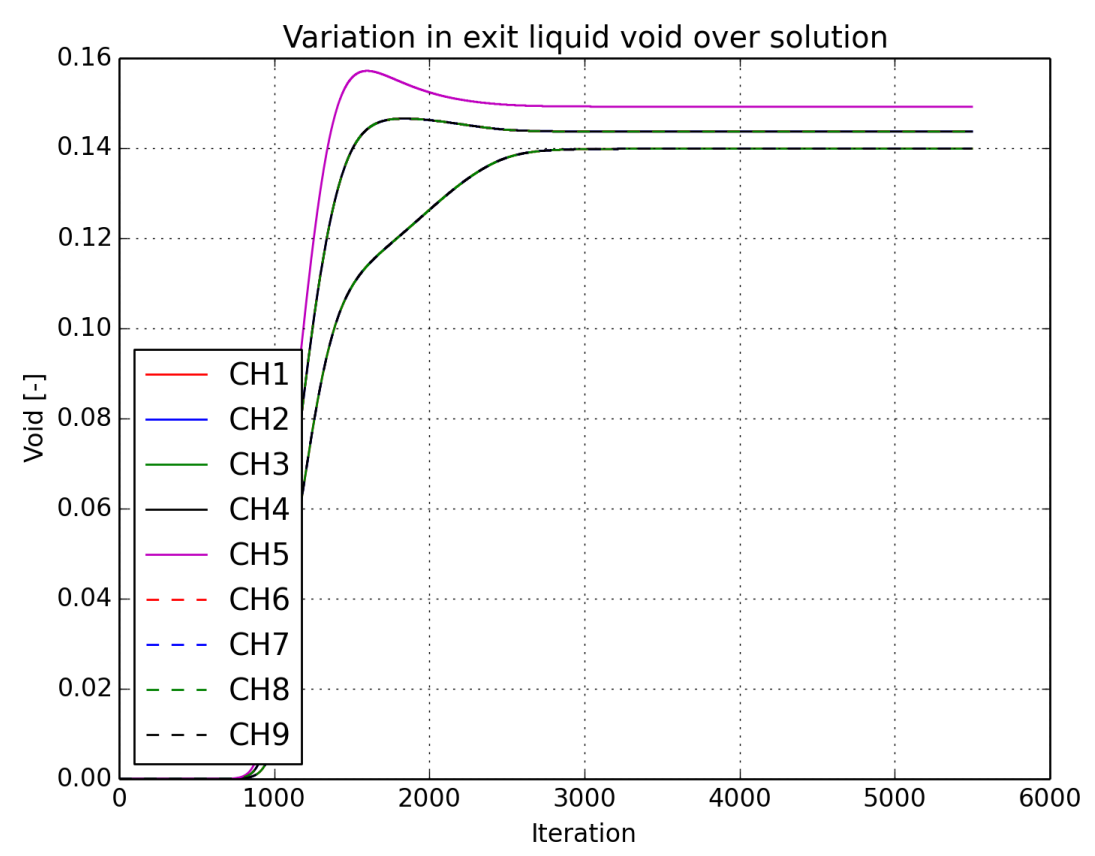

Figure 37. Exit void in Test 3 of $2 \times 2$ with outlet pressure set to 100 bar (medium void).

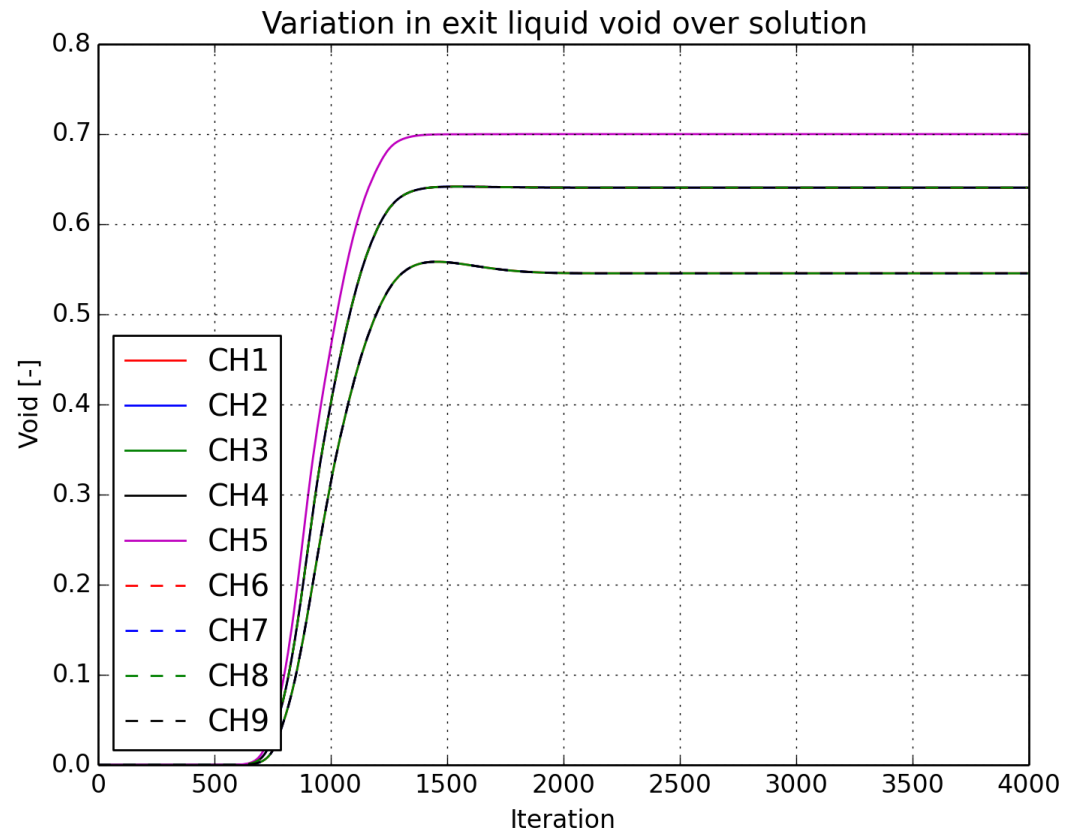

Figure 38. Exit void in Test 3 of $2 \times 2$ with outlet pressure set to 100 bar (high void). 


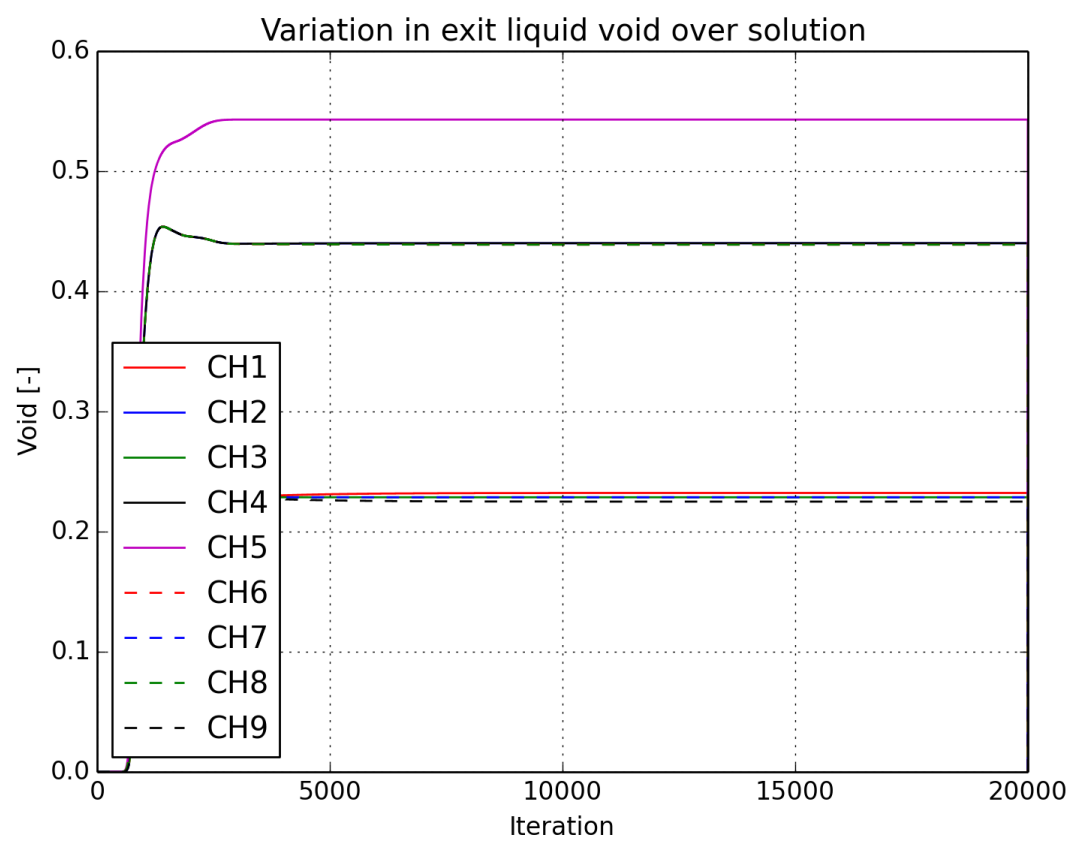

Figure 39. Exit void in Test 3 of $2 \times 2$ with outlet pressure set to 100 bar (bundle-average void around 0.4).

channel.

The axial flow distribution behavior starts to change near the top of the model. This is likely due to the occurrence of a flow regime transition. The CTF flow regime maps are primarily a function of void fraction, so as void migrates from one channel to another, the flow regime may change, which leads to different models being used for closure terms like interfacial drag and heat transfer. This leads to a new mechanical equilibrium point. Note that the flow regime is unique for each channel, so the bundle may have different flow regimes in different channels near the top of the facility.

While extending the facility has no impact on code convergence for the well-converging cases, extending the facility for certain void fractions exacerbates the convergence problems. Figure 42 shows what happens to the 100 bar, $40 \%$ void case when the facility is extended. Whereas the corner void was diverging only slightly in Figure 39, the void distribution is now completely chaotic when the facility is made longer.

This study shows that cases are generally easier to converge when pressure is higher; however, mixture enthalpy (void content) also has a significant impact on code convergence. There seems to be a range of void fractions that are more prone to causing numerical oscillations and solution divergence. This range grows smaller as the pressure is increased. This may indicate a problem with some of the closure relationships for a certain flow regime. The problem may be an actual bug or simply insufficient underrelaxation of the source term being calculated by the model. It is important to note, though, that the GE $3 \times 3$ facility was run at a wide range of void fractions that cover the problematic range in this study and no convergence issues were experienced for those cases. It is possible that the much larger geometry of the $2 \times 2$ facility (lattice dimensions are twice that of a normal BWR) may also have something to do with the convergence problems.

As a result of the extensive difficulties encountered in trying to model this facility, it is unclear if any meaningful conclusions can be drawn in support of validating the void drift model. The higher pressure cases were, for the most part, successful; however, it is shown that the CTF-predicted flow is not fully developed. Furthermore, it is not clear what uncertainty is introduced by artificially elevating the operating pressure. 


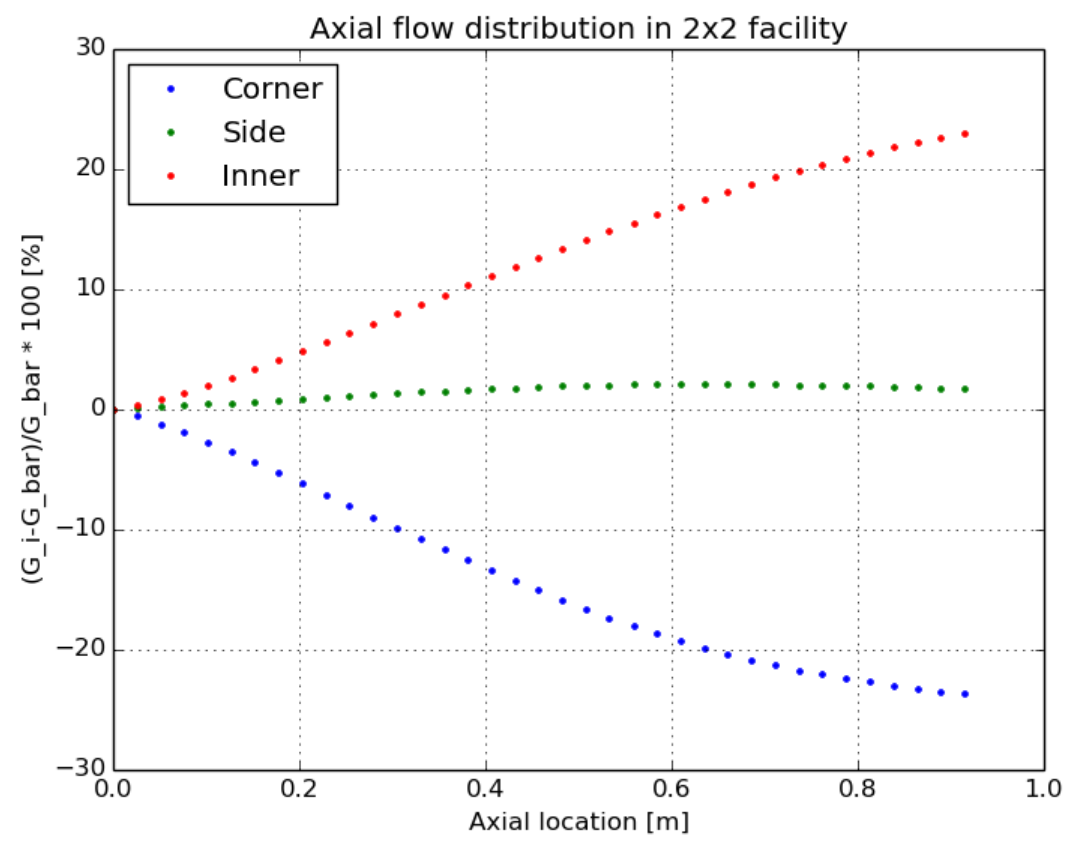

Figure 40. Normalized flow distribution in the three channel types of the $2 \times 2$ facility in CTF simulation run at 100 bar.

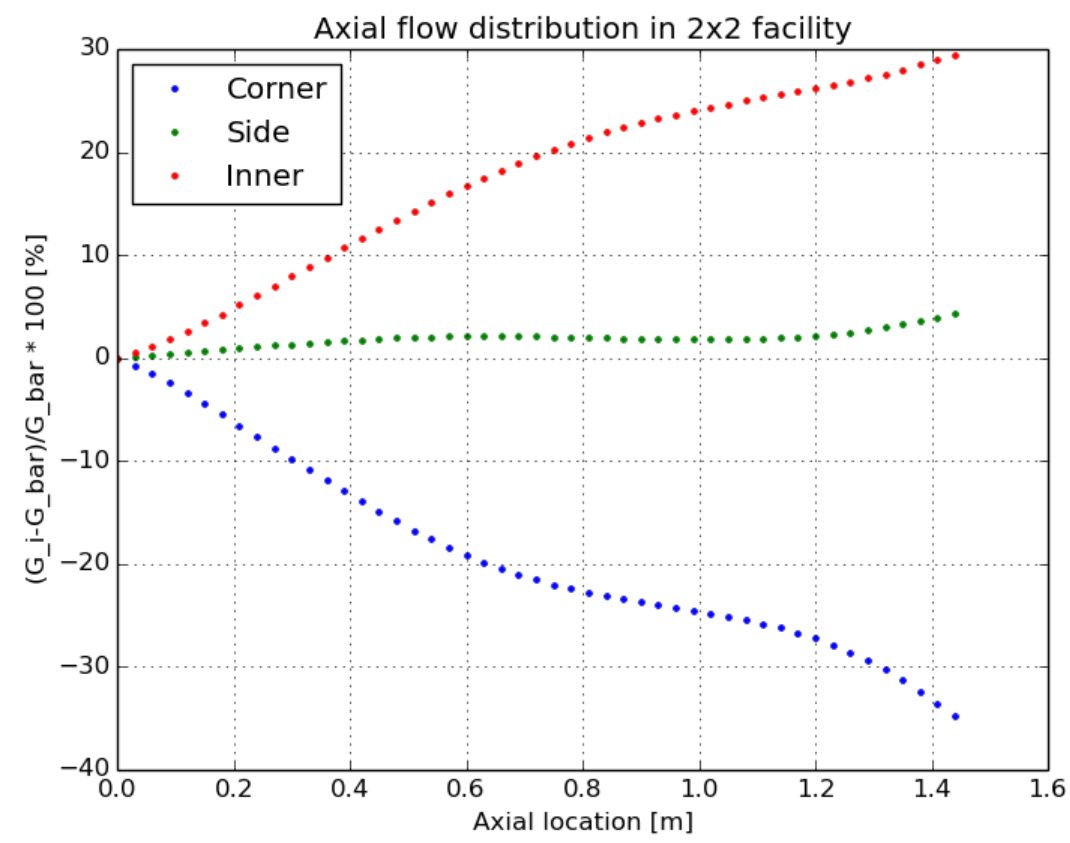

Figure 41. Normalized flow distribution in the three channel types of the $2 \times 2$ facility in CTF simulation run at $\mathbf{1 0 0}$ bar when facility is extended. 


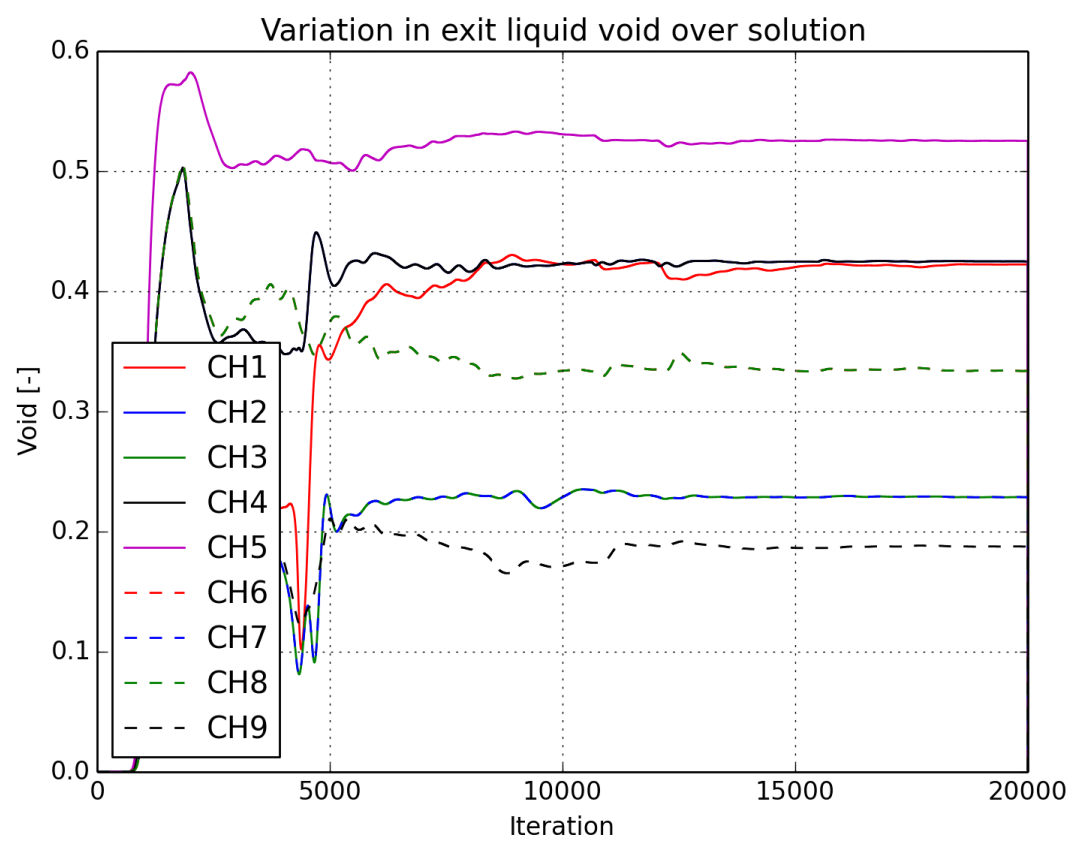

Figure $42.2 \times 2$ Test 3 run at 100 bar and $40 \%$ void when facility length is extended.

\subsection{GE $3 \times 3$ Validation}

The $3 \times 3$ facility was introduced in Section 2.5. The single-phase tests were modeled in that section, and it was demonstrated that CTF predicts the correct flow distribution for single-phase flow. The two-phase tests were modeled previously in the CTF Validation Manual [4]. This study expands on the previous validation work by including all test cases (some test cases were not included in the original validation work) and using the optimal mixing coefficient of 0.007 along with the effect of switching from Rogers \& Rosehart. Additionally, this study observes behavior of the axial mass flux distribution predicted by CTF rather than only the predicted outlet mass flux value.

CTF Model Description The base CTF model is described in Section 2.5. The geometry of all tests is the same, so only the boundary conditions need to be modified between runs. The process of generating the input decks, running them, and processing results is scripted so that sensitivity studies and automated testing may be performed easily. This study includes several perturbations of modeling choices of interest:

- The mixing model is changed between a constant of 0.007 and the Rogers \& Rosehart model.

- A case is run with void drift disabled.

- A case is run with no grids for observing axial flow behavior.

Discussion of Results A comparison of the measured and predicted exit equilibrium qualities is provided in Figure 43 for the base case model, which uses the void drift model with $K_{a}$ set to 1.4, the turbulent mixing model with $\beta_{\mathrm{sp}}$ set to 0.007 , and $\Theta_{M}$ set to 5.0. Figure 43 shows that most predicted exit qualities fall within experimental uncertainty. However, the values that seem to vary furthest from measured results are qualities in the corner type subchannel. Root-mean-square of the error (RMSE) (Equation 37) values for the three subchannel types are shown directly in the figure.

$$
\mathrm{RMSE}=\sqrt{\frac{1}{N} \Sigma_{i=1}^{N}\left(x_{\mathrm{ctf}, i}-x_{\mathrm{m}, i}\right)^{2}}
$$


We see that, on average, the corner channel quality prediction error is about double the inner and side type channel prediction error. Furthermore, quality in the corner channels is typically overpredicted by CTF. The side and inner type exit qualities, however, generally match the experimental values within the measurement uncertainty.

If the void drift model is disabled, comparisons with experimental data become much worse. Figure 44 shows the exit equilibrium quality compared with experimental measurements with the void drift model disabled. Corner channel root-mean-square of error (RMSE) increases from 0.044 to 0.121 and inner channel RMSE increases from 0.012 to 0.019 for exit quality.

In addition to the exit equilibrium quality, the authors also measured the exit mass flux of each individual subchannel. Results for the two-phase experiments are shown in Figure 45. The rRMSE for the two-phase experiments are larger than for the single-phase results shown in Figure 29. The corner is still the most poorly predicted of all channel types. Figure 46 shows the exit mass flux for the two-phase cases when void drift is disabled. The accuracy of both corner and inner channel type predictions degrades sharply. Similarly, mass flux rRMSE more than doubles for the corner channel type when the void drift model is disabled.

The original GE $3 \times 3$ study used the Rogers \& Rosehart correlation to set the single-phase mixing coefficient. The results are repeated here for all experiments in Figures 47 and 48 for mass flux and quality. Prediction of both measured quantities improves when using Rogers \& Rosehart over the constant $\beta$ value of 0.007 . Results indicate there is almost no difference in accuracy of the results between using the Rogers \& Rosehart correlation to calculate single-phase mixing coefficient or the constant value of 0.007 .

The axial mass flux distribution of each channel type is also investigated. It is discovered that the distributions are complicated, so the spacer grids are removed for the sake of clarifying two-phase and flow-regime dependant trends. The turbulent-mixing and void drift models are enabled, with the single-phase mixing coefficient being set to 0.007 with the Beus two-phase-mixing multiplier set to 5.0. The following plots show the normalized mass flux in each channel type, calculated as local mass flux minus bundle average mass flux and divided by bundle average mass flux. The measured normalized mass fluxes are shown with horizontal dashed lines. The measured value is only available at the outlet. Different colored lines are used to denote different channel types; red for corner, blue for side, and green for inner.

The plots also include the flow regime, denoted with colored regions in the plot; SP stands for single-phase, SB for small bubble, SL for slug, CH for churn, and AN for annular. The flow regimes often change at different axial locations in different channel types. The gray shaded regions show the length of this transition region. For example, the green "SB" region means that all channel types are in the small bubble flow regime. When the gray region begins, it means at least one of the channel types has transitioned to slug flow, but at least one channel type is still in small bubble. When the gray region ends, it means that all channels are in slug. The flow quality is shown for each channel type using dot-dashed lines, which corresponds to a secondary $y$-axis.

The high-flow, low-power cases, $2 \mathrm{C} 1$ and 2C2, are shown in Figures 49 and 50. The figures show that the corner mass flux tends to decrease and inner mass flux tends to increase until the flow quality starts to increase. At this point, the void drift effect begins to draw the normalized mass fluxes back toward zero. Test $2 \mathrm{C} 2$ has lower subcooling, so this effect starts sooner in the bundle. In Case $2 \mathrm{C} 2$, there is a "notch" in the axial mass flux profile for the corner channel. It is found that this is due to entrainment starting by running a case with the droplet field disabled.

The axial flow behavior is fairly smooth in the "C"-series cases compared with higher power cases and lower flow cases. Test $2 \mathrm{G} 1$ has the power doubled compared with the $2 \mathrm{C} 1$ and $2 \mathrm{C} 2$ cases. Its flow distribution is shown in Figure 51. Because of high subcooling, the exit flow quality is still only about $10 \%$, but the flow distributions look more chaotic starting at about the $0.8 \mathrm{~m}$ mark. The flow distribution appears to begin changing in the slug flow regime and transition to churn.

The "D"-series tests have the highest power-to-flow ratio. Test 2D3 makes it into the annular flow regime as a result. Its axial flow distribution is shown in Figure 52. Flow quality goes as high as almost $40 \%$. The flow distribution experiences a significant trend reversal at about $1.5 \mathrm{~m}$, as the channels transition into the annular flow regime. Again, there is a "notch" in the corner channel mass flux distribution due to the start of entrainment. 


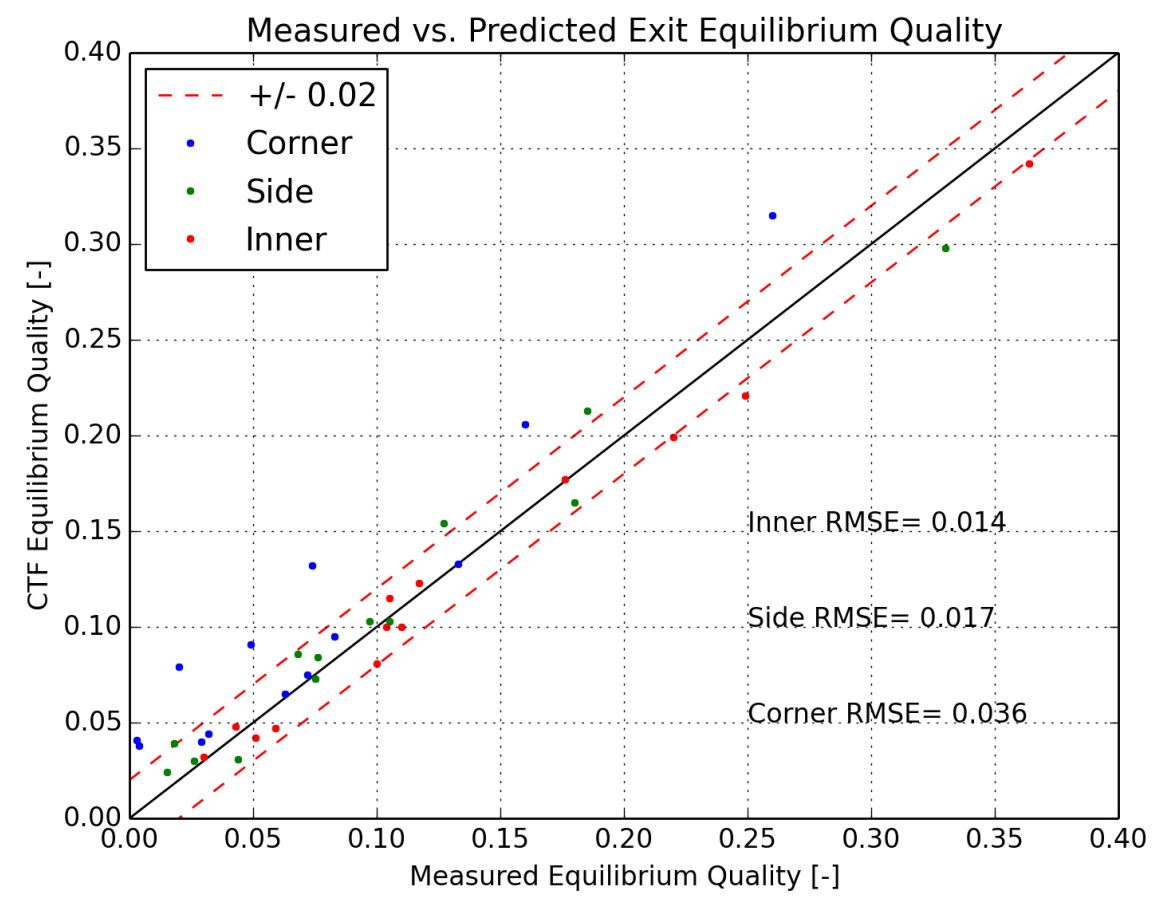

Figure 43. Comparison of predicted and measured exit equilibrium quality for GE $3 \times 3$ two-phase cases.

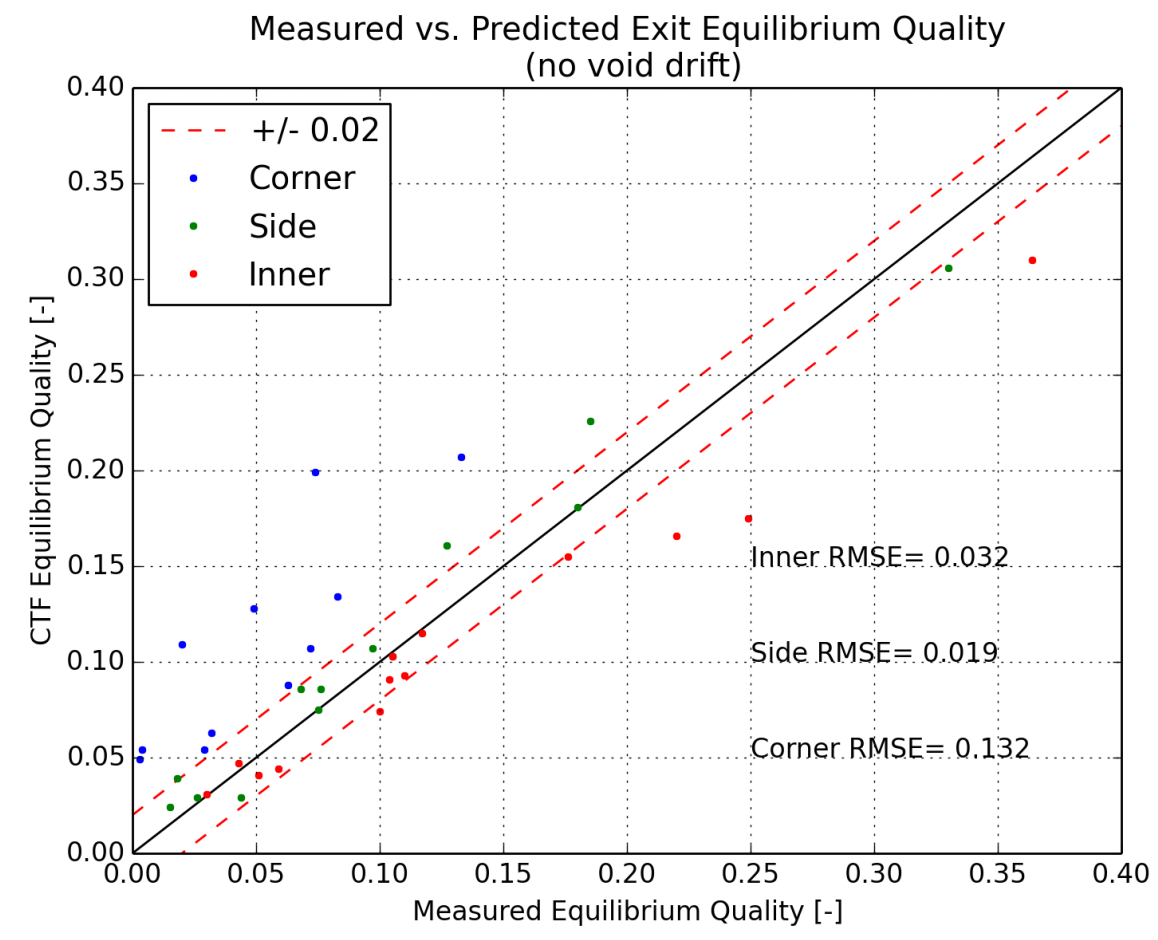

Figure 44. Comparison of predicted and measured exit equilibrium quality for GE $3 \times 3$ two-phase cases when void drift is disabled. 


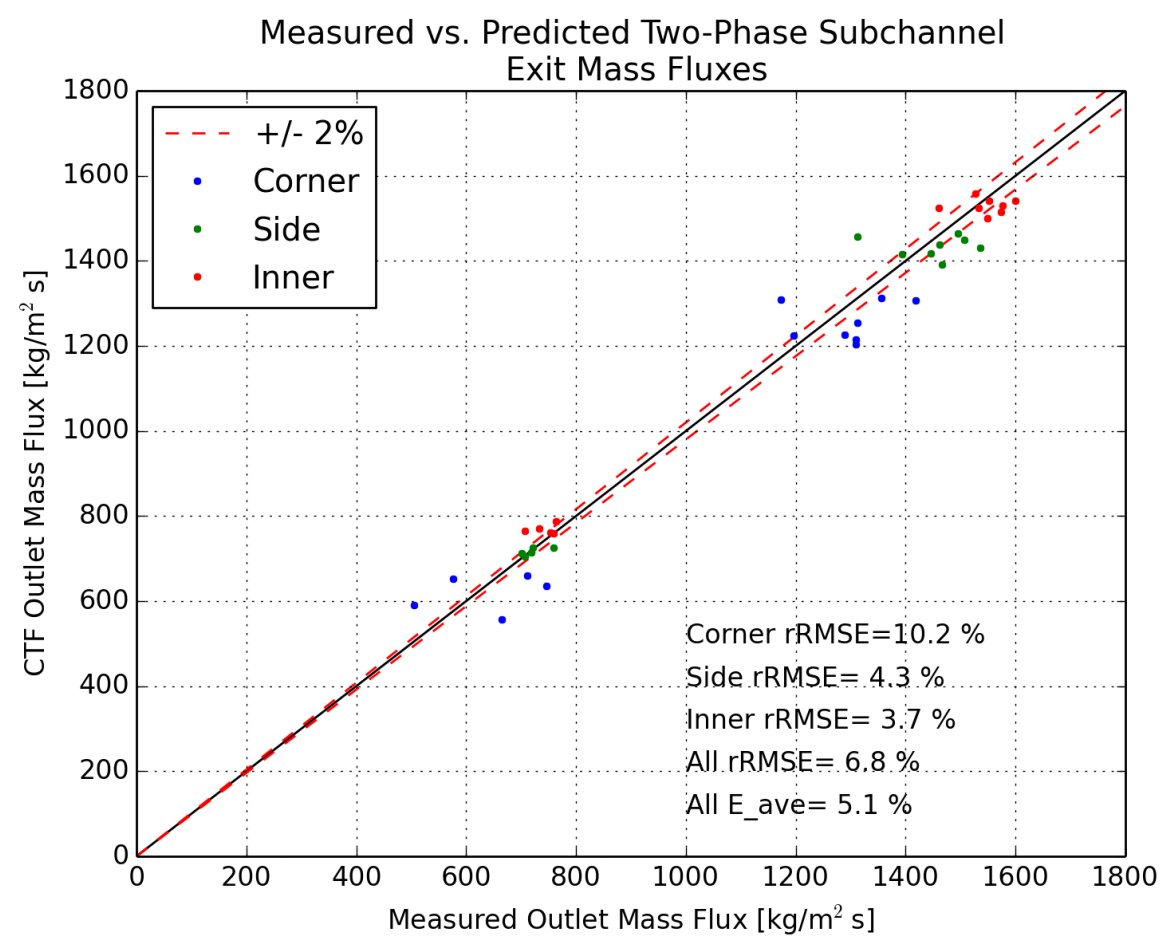

Figure 45. Comparison of predicted and measured exit mass flux for GE $3 \times 3$ two-phase cases.

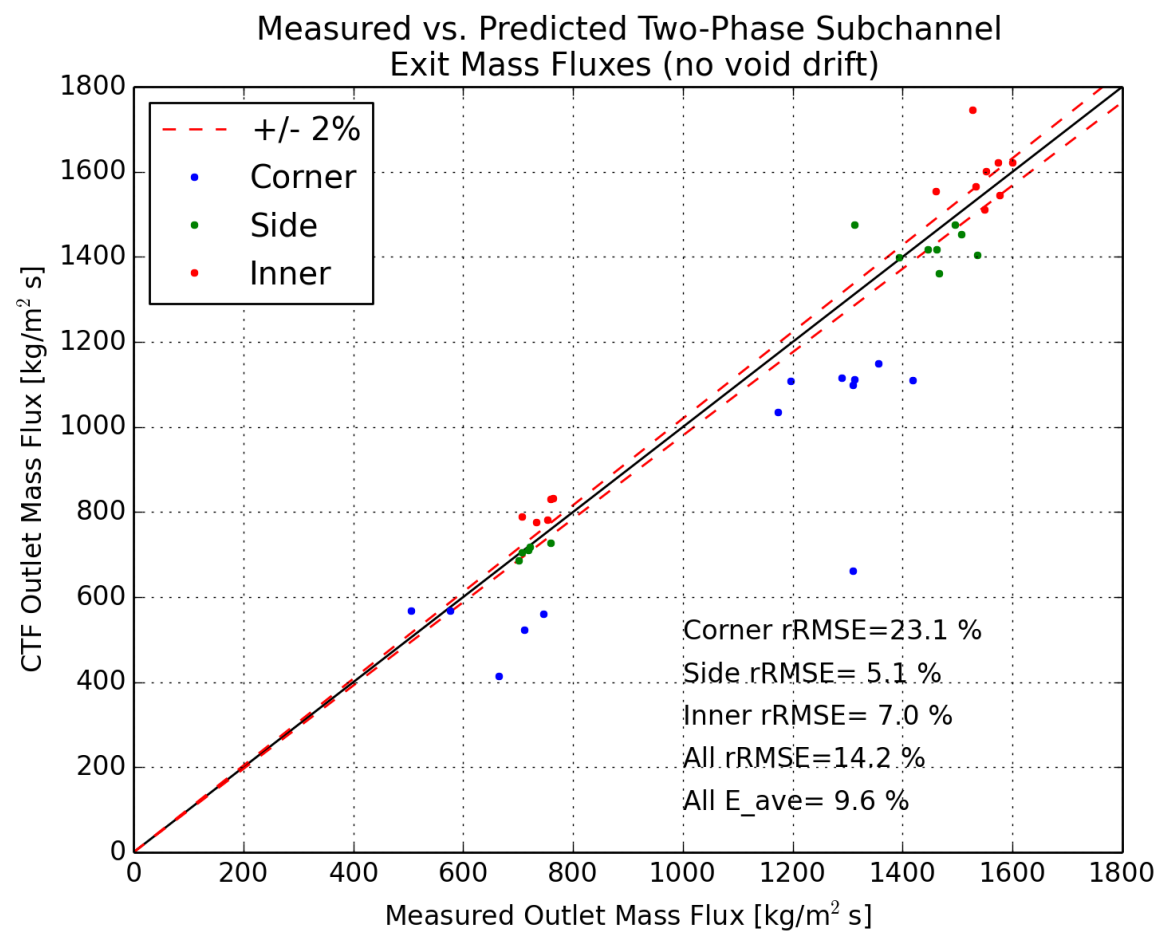

Figure 46. Comparison of predicted and measured exit mass flux for GE $3 \times 3$ two-phase cases when void drift is disabled. 


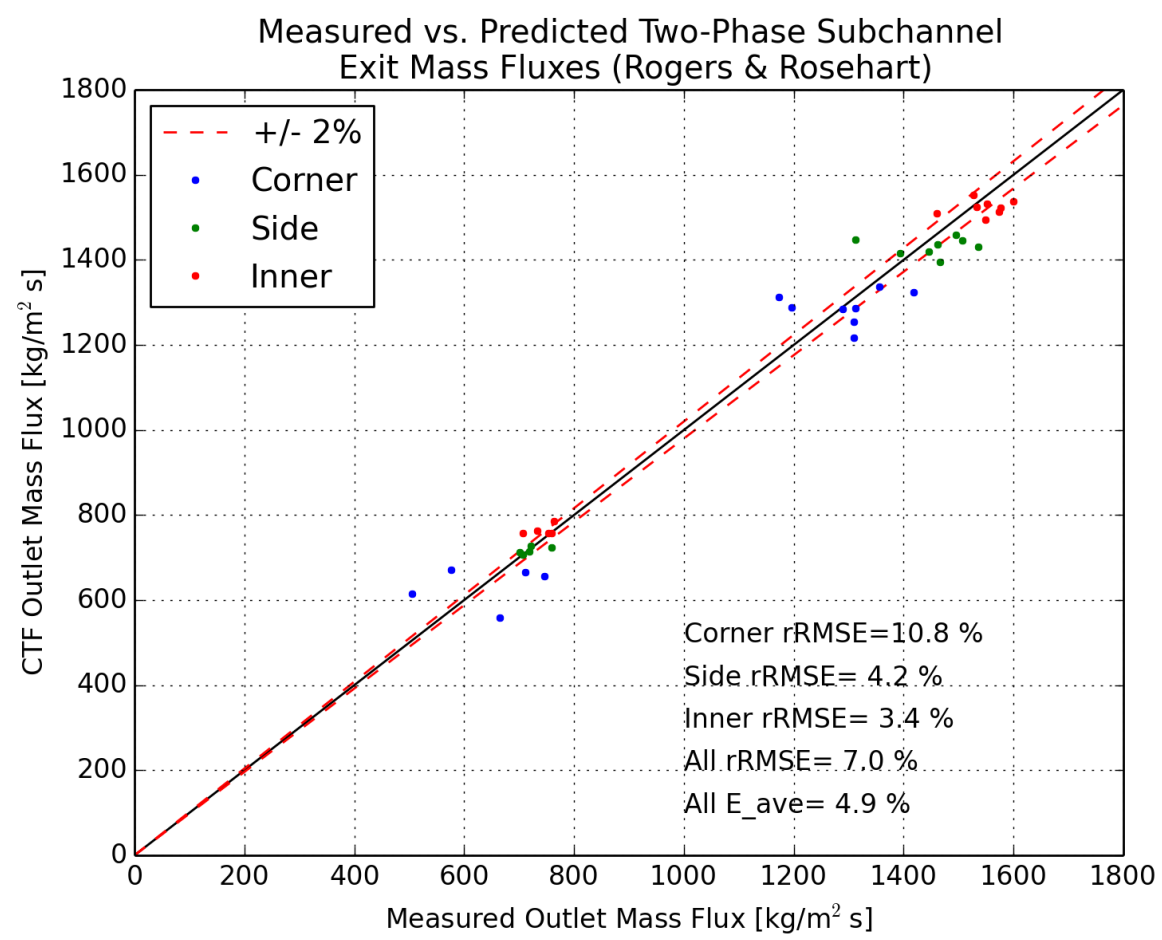

Figure 47. Comparison of predicted and measured exit mass flux for GE $3 \times 3$ two-phase cases using Rogers \& Rosehart.

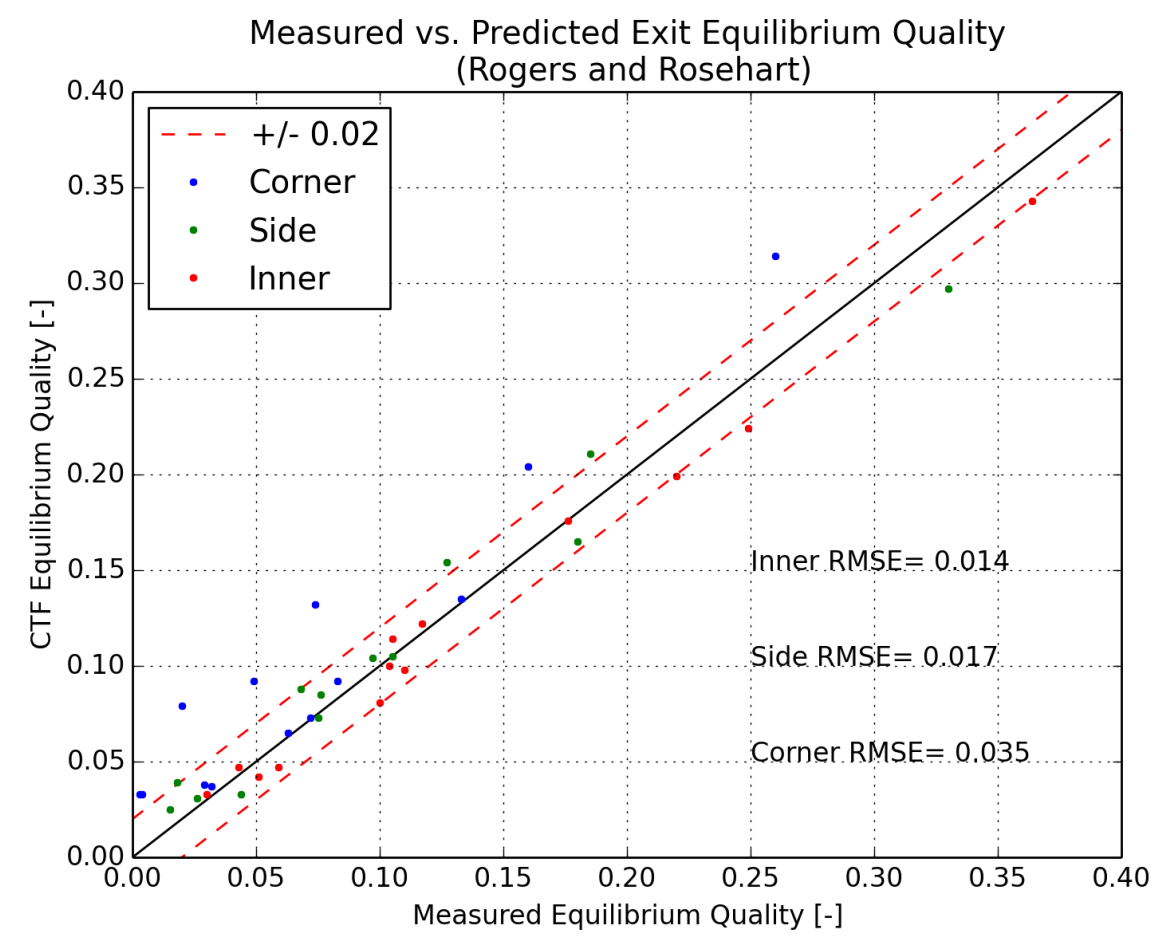

Figure 48. Comparison of predicted and measured exit quality for GE $3 \times 3$ two-phase cases using Rogers \& Rosehart. 
The overall trend seems for flow to migrate from the corner channel to the side and inner channels when flow is low void. At the start of bulk boiling, this trend reverses, causing channel flow to stay the same or move back to the average. The transition to the annular flow regime causes a second reversal, making flow migrate out of the corner channel and back into the inner and side channels due to a change in constitutive models. 


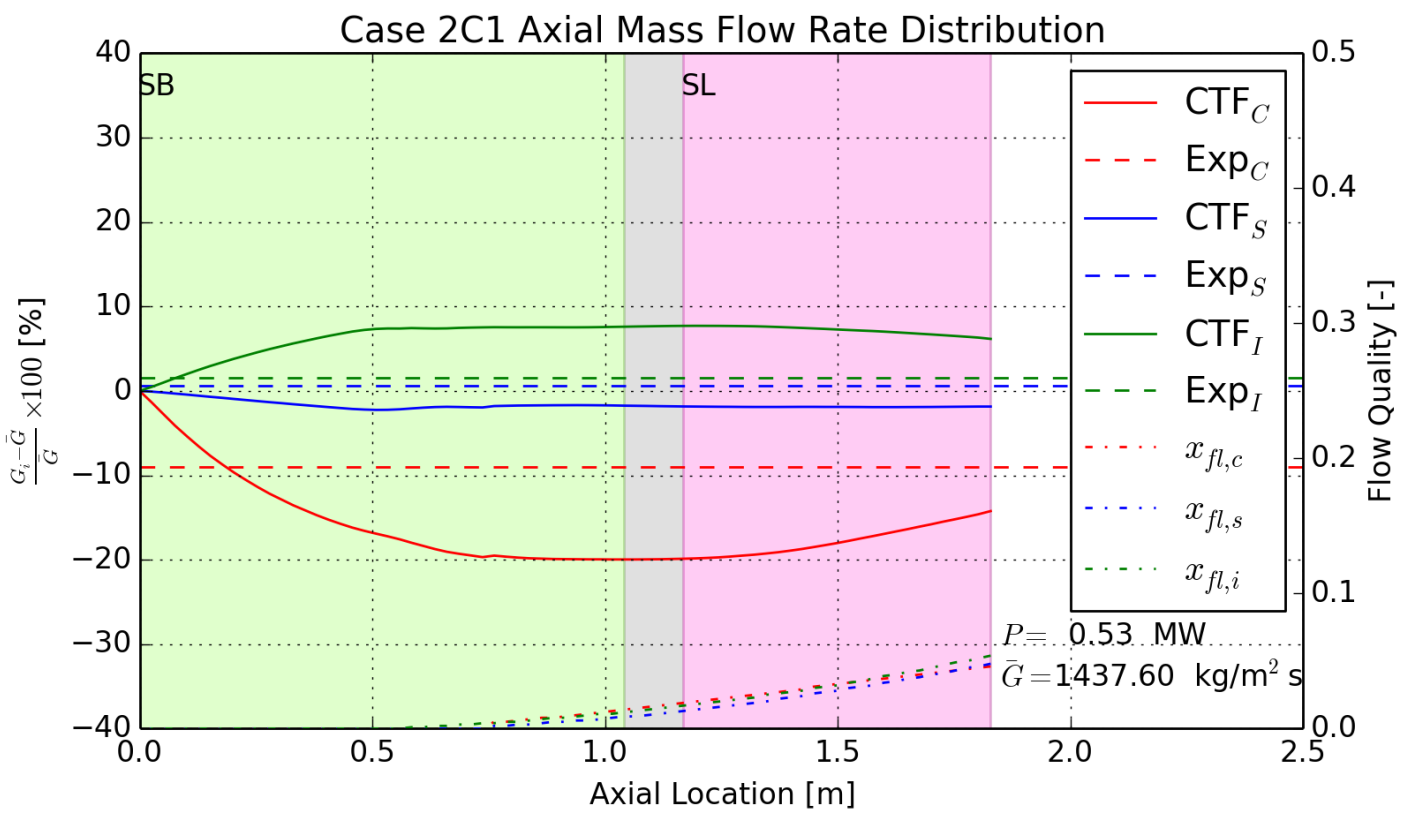

Figure 49. GE $3 \times 3$ Test $2 \mathrm{C} 1 \mathrm{CTF}$-predicted axial flow distribution and flow quality distribution compared with experimental measurement.

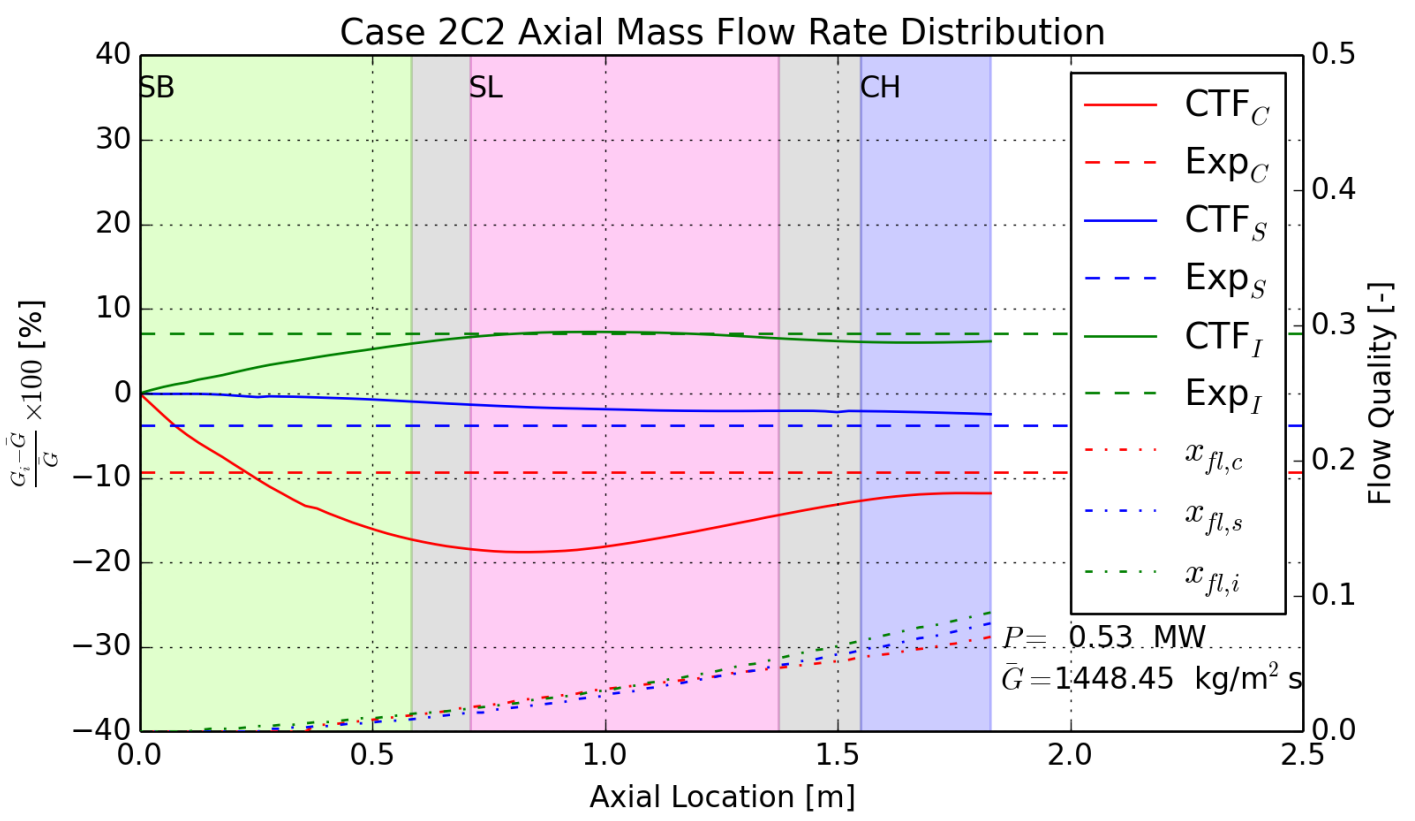

Figure 50. GE $3 \times 3$ Test 2 C2 CTF-predicted axial flow distribution and flow quality distribution compared with experimental measurement. 


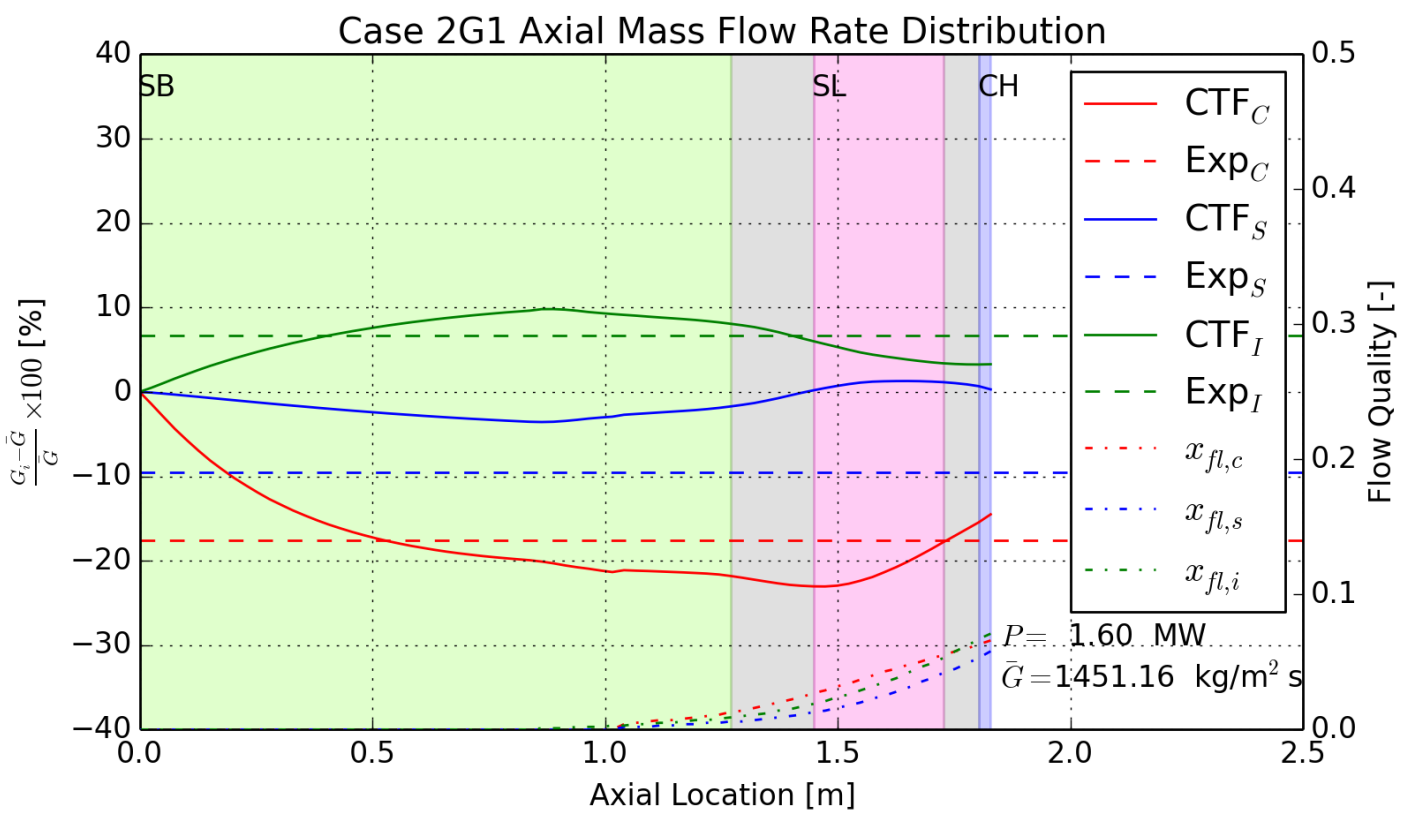

Figure 51. GE $3 \times 3$ Test 2 G1 CTF-predicted axial flow distribution and flow quality distribution compared with experimental measurement.

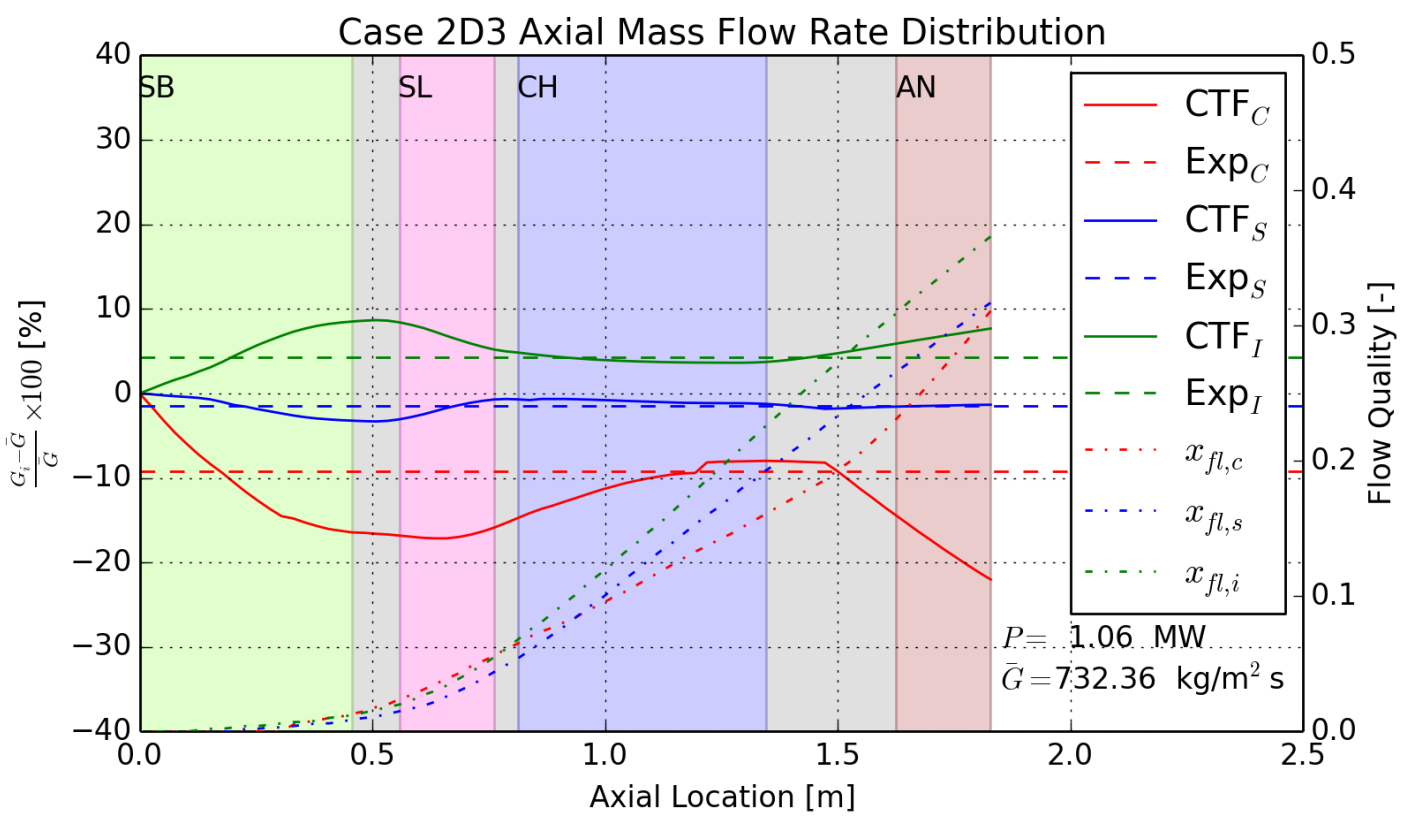

Figure 52. GE $3 \times 3$ Test 2D3 CTF-predicted axial flow distribution and flow quality distribution compared with experimental measurement. 


\subsection{BFBT $8 \times 8$ Validation}

Facility Description The BFBT tests are organized into phases and exercises as described in the BFBT specification [12].

- Phase I: Void Distribution Benchmark

- Exercise 1: Steady-state subchannel grade benchmark

- Exercise 2: Steady-state microscopic grade benchmark

- Exercise 3: Transient microscopic grade benchmark

- Exercise 4: Uncertainty analysis of the void distribution benchmark

- Phase II: Critical Power Benchmark

- Exercise 0: Steady-state pressure drop benchmark

- Exercise 1: Steady-state critical power benchmark

- Exercise 2: Transient benchmark

The CTF Validation document [4] includes tests from Phase II, Exercise 0. This validation study expands on the BFBT tests included in the CTF validation matrix by modeling Phase I, Exercise 1. For this validation study, 16 tests are modeled. Table 3 shows the tests and their operating conditions that are modeled as part of this study.

The assembly type refers to the placement of heater rods, guide tubes, the water rod, and power configurations. Figures 53 and 54 show the geometry and rod configuration for Assembly Types $0-1,0-2$, and $0-3$. All of these tests have a uniform axial and radial heating. The only difference between the assembly types is that some heater rods are shut off in Assembly Types 0-2 and 0-3. Figure 55 shows the geometry and power configuration for Assembly Types 1, 2, and 3. Only tests of Assembly Type 1 are modeled in this study. Assembly Type 1 has a cosine power shape, which is shown in Table 4. All other assembly types modeled in this study have a uniform axial power shape. Assembly Types 1 and 4 have non-uniform radial power distributions. The Assembly Type 1 radial power distribution is shown in Figure 56, and the Assembly Type 4 radial power distribution is shown in Figure 57.

Finally, Figure 58 shows the rod configuration and power distribution for Assembly Types 4, C2A, C2B, and C3. Only tests of Assembly Type 4 are included in this study. Assembly Type 4 replaces the four central rods with a large water rod.

Void measurements were made in the facility using two approaches: 1) an x-ray densitometer was used at several axial locations, and 2) a CT scanner was used to obtain a fine-mesh void distribution measurement $50 \mathrm{~mm}$ above the end of heated length. The CT scanner measurements are used for comparison with CTF in this study. Figure 59 shows a schematic of where different void measurements were made in the facility.

The CT scanner worked by sweeping over the bundle at a fixed axial plane to obtain void measurements in small "pixels" as small as $0.3 \times 0.3 \mathrm{~mm}$. The refined measurements have been used for CFD validation work, but the measurements were also post-processed to create a set of subchannel-averaged void measurements for comparison with subchannel codes. The measurements were made several times for the same operating conditions and were time-averaged to avoid the effects of two-phase flow oscillations.

The BFBT specification [12] states that the subchannel accuracy is estimated to be $3 \%$ and the bundle average void is estimated to be $2 \%$ (as will be shown when analyzing symmetry of the results, the measurement error is likely much higher than this).

CTF Model Figure 60 shows the flow area and wetted perimeter for the channels in the CTF model for Assembly Types 0-1, 0-2, 0-3, and 1. It also shows the channel indexing scheme employed in the model. Note that the center channel is indexed as Channel 81. For the Assembly Type 4, Channel 81 is simply removed from the model along with the four surrounding rods. A large water rod is positioned in their place and the flow areas are modified as shown in Figure 61. 
Table 3. BFBT void distribution test conditions

\begin{tabular}{crrrrr}
\hline Test & Assembly Type & $\begin{array}{r}\text { Pressure } \\
{[\mathrm{MPa}]}\end{array}$ & $\begin{array}{r}\text { Inlet Subcooling } \\
{[\mathrm{kJ} / \mathrm{kg}]}\end{array}$ & $\begin{array}{r}\text { Flow Rate } \\
{[\mathrm{ton} / \mathrm{h}]}\end{array}$ & $\begin{array}{r}\text { Power } \\
\text { MW }\end{array}$ \\
\hline $0011-55$ & $0-1$ & 7.180 & 52.60 & 54.03 & 1.90 \\
$0011-58$ & $0-1$ & 7.172 & 51.00 & 54.90 & 3.51 \\
$0011-61$ & $0-1$ & 7.210 & 50.90 & 54.79 & 6.44 \\
$0021-16$ & $0-2$ & 7.190 & 54.00 & 54.85 & 1.91 \\
$0021-18$ & $0-2$ & 7.171 & 49.80 & 54.90 & 3.51 \\
$0021-21$ & $0-2$ & 7.179 & 51.40 & 54.90 & 6.45 \\
$0031-16$ & $0-3$ & 7.180 & 52.40 & 54.96 & 1.92 \\
$0031-18$ & $0-3$ & 7.179 & 50.00 & 54.79 & 3.52 \\
$0031-21$ & $0-3$ & 7.171 & 49.40 & 54.90 & 6.45 \\
$1071-55$ & 1 & 7.191 & 52.80 & 54.61 & 1.92 \\
$1071-58$ & 1 & 7.158 & 50.30 & 55.07 & 3.52 \\
$1071-61$ & 1 & 7.200 & 51.80 & 54.65 & 6.48 \\
$4101-53$ & 4 & 7.181 & 52.80 & 54.65 & 1.24 \\
$4101-55$ & 4 & 7.195 & 52.90 & 54.59 & 1.92 \\
$4101-58$ & 4 & 7.152 & 50.60 & 54.58 & 3.52 \\
$4101-61$ & 4 & 7.180 & 52.50 & 54.65 & 6.48 \\
\hline
\end{tabular}

\begin{tabular}{|c|c|c|c|}
\hline Item & \multicolumn{3}{|c|}{ Data } \\
\hline Assembly & 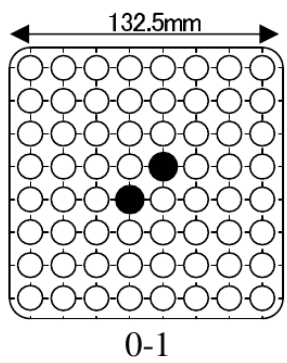 & 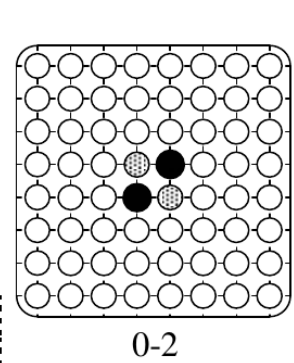 & 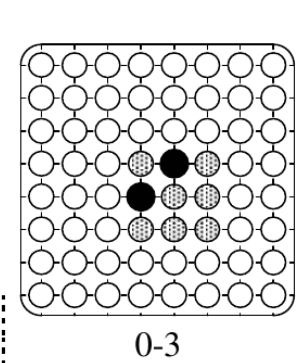 \\
\hline Simulated fuel assembly type & & $8 \times 8$ & 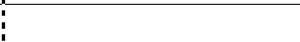 \\
\hline Number of heated rods & 62 & 60 & 55 \\
\hline Number of unheated rods & 0 & 2 & 7 \\
\hline Heated rods outer diameter $(\mathrm{mm})$ & & 12.3 & \\
\hline Heated rods pitch $(\mathrm{mm})$ & & 16.2 & \\
\hline Axial heated length (mm) & & 3708 & \\
\hline
\end{tabular}

O Heated rod, () Unheated rod, $\bullet$ Water rod - no flow in water rods

Figure 53. Description of BFBT Assembly Types 0-1, 0-2, and 0-3 (reprinted from B. Neykov et al. NUPEC BWR Full-size Fine-mesh Bundle Test (BFBT) Benchmark. Tech. rep. Nuclear Energy Agency, 2006) 


\begin{tabular}{|l|c||}
\hline \multicolumn{1}{|c|}{ Item } & Data \\
\hline Number of water rods & 2 \\
\hline Water rods outer diameter $(\mathrm{mm})$ & 15.0 \\
\hline Channel box inner width $(\mathrm{mm})$ & 132.5 \\
\hline Channel box corner radius $(\mathrm{mm})$ & 8.0 \\
\hline In channel flow area $\left(\mathrm{mm}^{2}\right)$ & 9781 \\
\hline Spacer type & Grid \\
\hline Number of spacers & 7 \\
\hline Spacer pressure loss coefficients & 1.2 \\
\hline \multirow{2}{*}{ Spacer location $(\mathrm{mm})$} & 455, 967, 1 479, 1991, 2 503, 3 015, 3 527 \\
\hline Radial power shape & Uniform \\
\hline Axial power shape & Uniform \\
\hline
\end{tabular}

Figure 54. Description of BFBT Assembly Types 0-1, 0-2, and 0-3 (continued) (reprinted from B. Neykov et al. NUPEC BWR Full-size Fine-mesh Bundle Test (BFBT) Benchmark. Tech. rep. Nuclear Energy Agency, 2006).

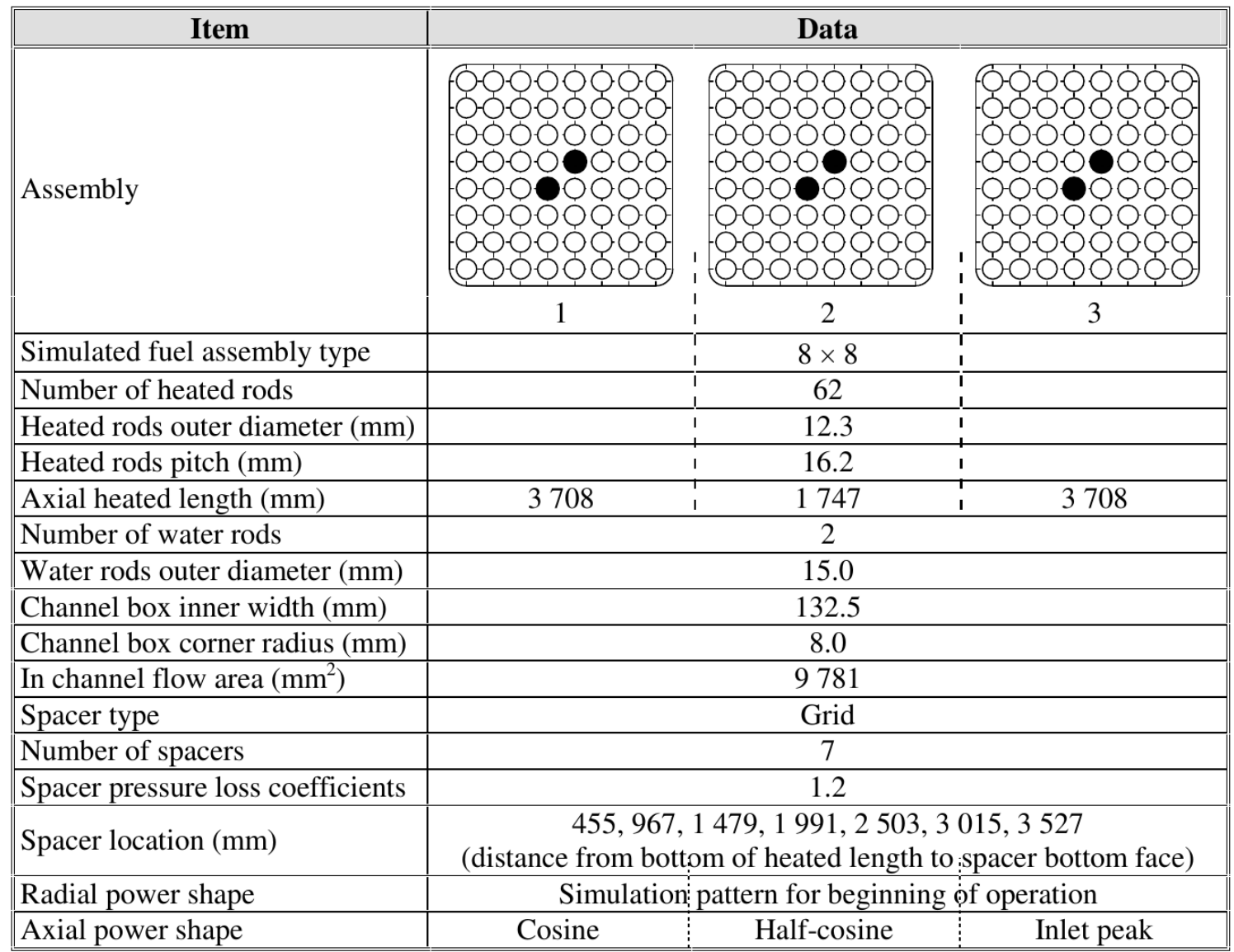

O Heated rod, $\rightarrow$ Unheated rod, $\bullet$ Water rod - no flow in water rods

Figure 55. Description of BFBT Assembly Types 1, 2, and 3 (reprinted from B. Neykov et al. NUPEC BWR Full-size Fine-mesh Bundle Test (BFBT) Benchmark. Tech. rep. Nuclear Energy Agency, 2006). 
Table 4. BFBT Assembly Type 1 axial power distribution.

\begin{tabular}{cr}
\hline Axial level & Power factor \\
\hline 1 & 0.46 \\
2 & 0.58 \\
3 & 0.69 \\
4 & 0.79 \\
5 & 0.88 \\
6 & 0.99 \\
7 & 1.09 \\
8 & 1.22 \\
9 & 1.22 \\
10 & 1.34 \\
11 & 1.34 \\
12 & 1.40 \\
13 & 1.40 \\
14 & 1.34 \\
15 & 1.34 \\
16 & 1.22 \\
17 & 1.22 \\
18 & 1.09 \\
19 & 0.99 \\
20 & 0.88 \\
21 & 0.79 \\
22 & 0.69 \\
23 & 0.58 \\
24 & 0.46 \\
\hline &
\end{tabular}

\begin{tabular}{|l:l:l:l:l:l:l:l:}
\hline 1.15 & 1.30 & 1.15 & 1.30 & 1.30 & 1.15 & 1.30 & 1.15 \\
\hline 1.30 & 0.45 & 0.89 & 0.89 & 0.89 & 0.45 & 1.15 & 1.30 \\
\hline 1.15 & 0.89 & 0.89 & 0.89 & 0.89 & 0.89 & 0.45 & 1.15 \\
\hline 1.30 & 0.89 & 0.89 & 0.89 & & 0.89 & 0.89 & 1.15 \\
\hline 1.30 & 0.89 & 0.89 & & 0.89 & 0.89 & 0.89 & 1.15 \\
\hline 1.15 & 0.45 & 0.89 & 0.89 & 0.89 & 0.89 & 0.45 & 1.15 \\
\hline 1.30 & 1.15 & 0.45 & 0.89 & 0.89 & 0.45 & 1.15 & 1.30 \\
\hline 1.15 & 1.30 & 1.15 & 1.15 & 1.15 & 1.15 & 1.30 & 1.15 \\
\hline
\end{tabular}

Figure 56. Radial power distribution applied to Assembly Type 1 (reprinted from B. Neykov et al. NUPEC BWR Full-size Fine-mesh Bundle Test (BFBT) Benchmark. Tech. rep. Nuclear Energy Agency, 2006). 


\begin{tabular}{|l|l|l|l|l|l|l|l|}
\hline 1.15 & 1.30 & 1.15 & 1.30 & 1.30 & 1.15 & 1.30 & 1.15 \\
\hline 1.30 & 0.45 & 0.89 & 0.89 & 0.89 & 0.45 & 1.15 & 1.30 \\
\hline 1.15 & 0.89 & 0.89 & 0.89 & 0.89 & 0.89 & 0.45 & 1.15 \\
\hline 1.30 & 0.89 & 0.89 & & & 0.89 & 0.89 & 1.15 \\
\hline 1.30 & 0.89 & 0.89 & & & 0.89 & 0.89 & 1.15 \\
\hline 1.15 & 0.45 & 0.89 & 0.89 & 0.89 & 0.89 & 0.45 & 1.15 \\
\hline 1.30 & 1.15 & 0.45 & 0.89 & 0.89 & 0.45 & 1.15 & 1.30 \\
\hline 1.15 & 1.30 & 1.15 & 1.15 & 1.15 & 1.15 & 1.30 & 1.15 \\
\hline
\end{tabular}

Figure 57. Radial power distribution applied to Assembly Type 4 (reprinted from B. Neykov et al. NUPEC BWR Full-size Fine-mesh Bundle Test (BFBT) Benchmark. Tech. rep. Nuclear Energy Agency, 2006).

\begin{tabular}{|c|c|c|c|c|}
\hline Item & \multicolumn{3}{|r|}{ Data } & \multirow[b]{2}{*}{$\begin{array}{l}1 \\
1 \\
1 \\
1 \\
1 \\
1 \\
1 \\
1 \\
1 \\
1\end{array}$} \\
\hline \multirow[t]{2}{*}{ Test assembly } & & $\begin{array}{c}\vdots \\
\vdots \\
\vdots \\
\vdots \\
\vdots\end{array}$ & 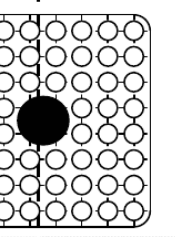 & \\
\hline & 4 & $\mathrm{C} 2 \mathrm{~A}$ & $\mathrm{C} 2 \mathrm{~B}$ & $\mathrm{C} 3$ \\
\hline Simulated fuel assembly type & \multicolumn{4}{|c|}{ High burn-up $8 \times 8$} \\
\hline Number of heated rods & \multicolumn{4}{|c|}{60} \\
\hline Heated rods outer diameter $(\mathrm{mm})$ & \multicolumn{4}{|c|}{12.3} \\
\hline Heated rods pitch $(\mathrm{mm})$ & \multicolumn{4}{|c|}{16.2} \\
\hline Axial heated length (mm) & \multicolumn{4}{|c|}{3708} \\
\hline Number of water rods & \multicolumn{4}{|c|}{1} \\
\hline Water rods outer diameter $(\mathrm{mm})$ & \multicolumn{4}{|c|}{34.0} \\
\hline Channel box inner width (mm) & \multicolumn{4}{|c|}{132.5} \\
\hline Channel box corner radius (mm) & \multicolumn{4}{|c|}{8.0} \\
\hline In channel flow area $\left(\mathrm{mm}^{2}\right)$ & \multicolumn{4}{|c|}{9463} \\
\hline Spacer type & \multicolumn{4}{|c|}{ Ferrule } \\
\hline Number of spacers & \multicolumn{4}{|c|}{7} \\
\hline Spacer pressure loss coefficients & \multicolumn{4}{|c|}{1.2} \\
\hline Spacer location $(\mathrm{mm})$ & \multicolumn{4}{|c|}{$\begin{array}{r}45: 5,967,1479,991,2503,3015,3527 \\
\text { (distance from bottom of heated length to spacer bottom face) }\end{array}$} \\
\hline Radial power shape & A & A & $\mathrm{B}$ & A \\
\hline Axial power shape & Uniform & Cosine & Cosine & Inlet-peak \\
\hline
\end{tabular}

O Heated rod, $\bigcirc$ Water rod - no flow in water rods

A: Simulation pattern for beginning of operation.

B: Simulation pattern for middle of operation.

Figure 58. Description of BFBT Assembly Type 4 (reprinted from B. Neykov et al. $N U$ PEC BWR Full-size Fine-mesh Bundle Test (BFBT) Benchmark. Tech. rep. Nuclear Energy Agency, 2006). 


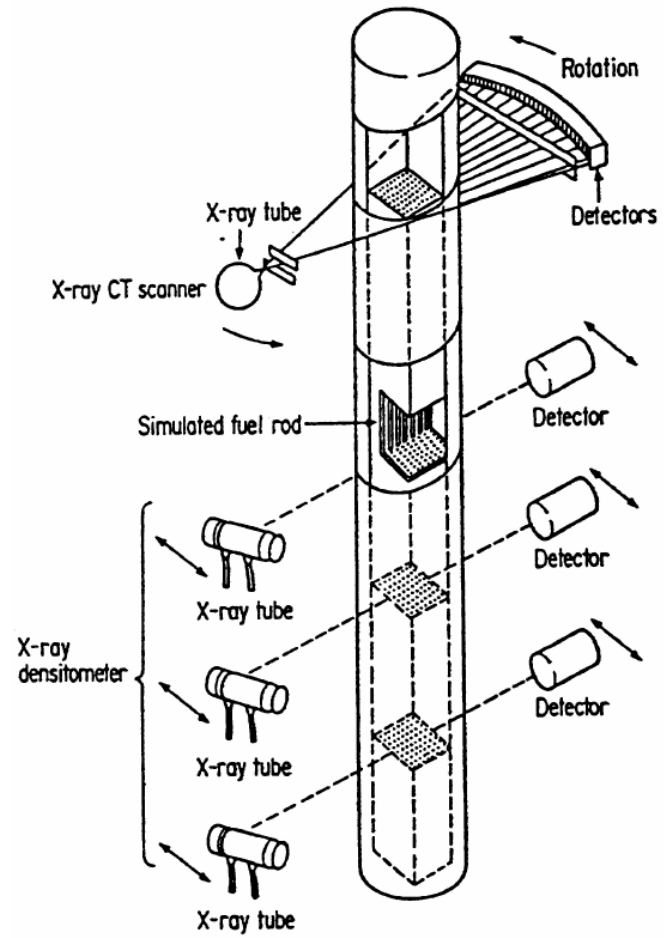

Figure 59. Depiction of void measurement locations and techniques in BFBT facility (reprinted from B. Neykov et al. NUPEC BWR Full-size Fine-mesh Bundle Test (BFBT) Benchmark. Tech. rep. Nuclear Energy Agency, 2006.

\begin{tabular}{|c|c|c|c|c|c|c|c|c|}
\hline 1 & 2 & 3 & 4 & 5 & 6 & 7 & 8 & 9 \\
\hline 10 & 11 & 12 & 13 & 14 & 15 & 16 & 17 & 18 \\
\hline 19 & 20 & 21 & 22 & 23 & 24 & 25 & 26 & 27 \\
\hline 28 & 29 & 30 & 31 & 32 & 33 & 34 & 35 & 36 \\
\hline 37 & 38 & 39 & 40 & 81 & 41 & 42 & 43 & 44 \\
\hline 45 & 46 & 47 & 48 & 49 & 50 & 51 & 52 & 53 \\
\hline 54 & 55 & 56 & 57 & 58 & 59 & 60 & 61 & 62 \\
\hline 63 & 64 & 65 & 66 & 67 & 68 & 69 & 70 & 71 \\
\hline 72 & 73 & 74 & 75 & 76 & 77 & 78 & 79 & 80 \\
\hline
\end{tabular}

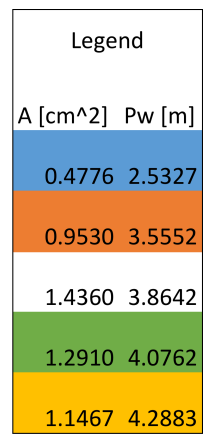

Figure 60. Map of the channel flow areas and wetted perimeters for Assembly Types $0-1,0-2,0-3$, and 1 . 


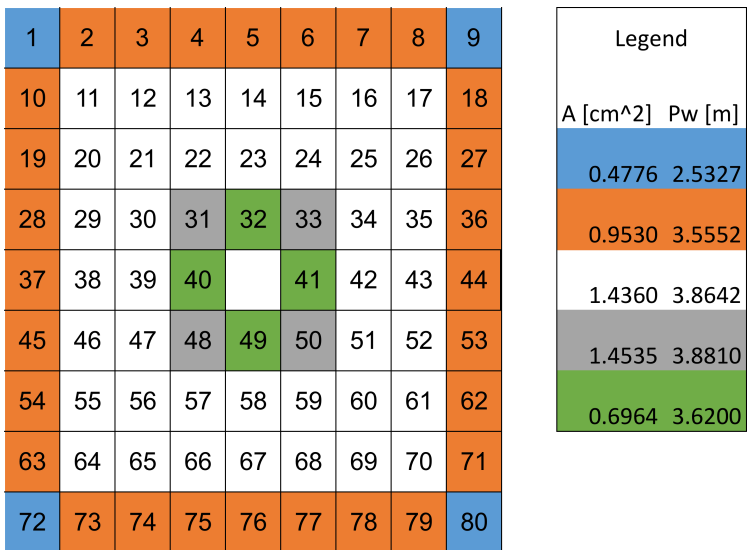

Figure 61. Map of the channel flow areas and wetted perimeters for Assembly Type 4.

Spacer grid locations are provided in Figures 53, 54, 55, and 58. These are ferrule-type spacers with circular tubes (ferrules) to guide each heated rod as well as the central water rod. An example of this type of grid is shown in Figure 62.

The loss coefficients for this type of spacer grid are calculated using the approach of Shiralkar and Radcliffe [13]. Details of the calculation are shown in [14]. Figure 63 shows the radial loss coefficient distribution in the bundle.

The CTF friction correlation is used [2] along with a single-phase mixing coefficient of 0.007 and a Beus two-phase multiplier, $\Theta_{M}$, of 5.0. The cases are run once with the droplet field enabled and once with the droplet field disabled to test the effect. When the droplet field is disabled, it means that there is no entrainment or de-entrainment and the interfacial drag and heat transfer between droplets and vapor is eliminated in the solution. It is discovered that the droplet field causes minor convergence problems and degrades the accuracy of results for all channel types, so the majority of results are presented with the droplet field disabled.

The axial mesh is set up so that the height of a spacer grid occupies one full level. The average axial mesh cell height is $3.635 \mathrm{~cm}$. Only heater rods are explicitly modeled; unheated objects, such as guide tubes and the fuel canister, are not modeled, as they have no impact on the solution in a steady-state solve. Furthermore, the power is set as a heat flux boundary condition, which disables the internal rod temperature solution.

Discussion of Results The process of running the simulations, extracting data from CTF output files, and creating plots is scripted for automated regeneration of analysis results. Most of the cases run by CTF converged to within tolerance. Convergence is checked by checking a collection of engineering parameters of interest:

1. Global energy balance (energy into the system minus energy out),

2. Energy movement between fields,

3. Global mass balance (mass into the system minus mass out),

4. Mass movement between fields due to evaporation/condensation,

5. Fluid energy storage between time steps,

6. Solid energy storage between time steps, and

7. Mass storage between time steps. 

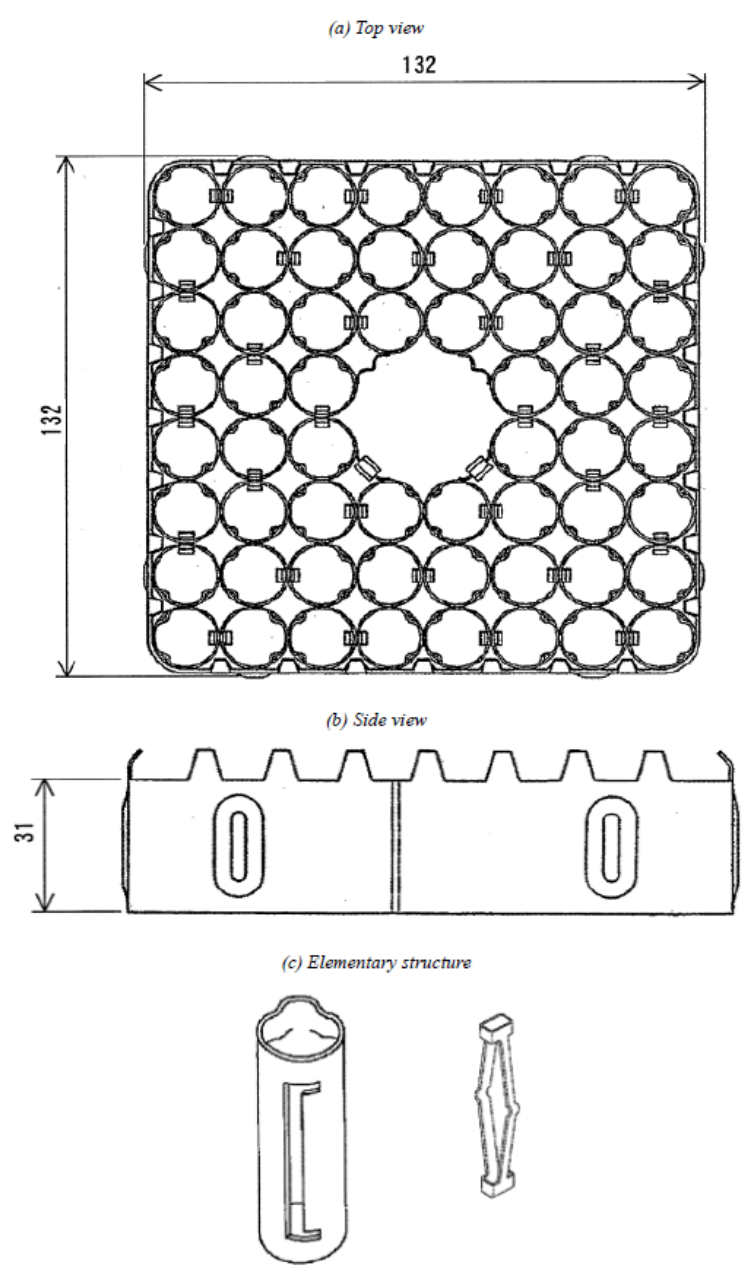

Figure 62. BFBT C2A ferrule grid geometry (reprinted from B. Neykov et al. NUPEC BWR Full-size Fine-mesh Bundle Test (BFBT) Benchmark. Tech. rep. Nuclear Energy Agency, 2006). 


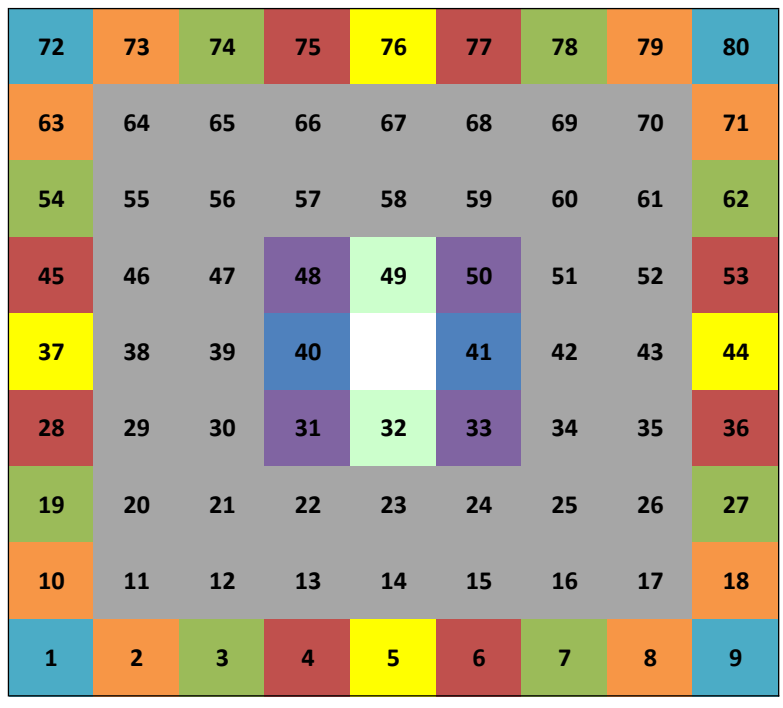

\begin{tabular}{|c|c|c|c|}
\hline \multicolumn{4}{|l|}{ Legend: } \\
\hline$k=1.348$ & $k=1.606$ & $k=1.304$ & $k=0.778$ \\
\hline$k=1.278$ & $k=1.222$ & $k=0.748$ & $k=0.856$ \\
\hline$k=0.926$ & & & \\
\hline
\end{tabular}

Figure 63. Map of channels in BFBT cases with loss coefficient for each channel as calculated by Shiralkar and Radcliffe (B. Shiralkar and D. Radcliffe. An Experimental and Analytical Study of the Synthesis of Grid Spacer Loss Coefficients. Tech. rep. NEDE-13181. General Electric, 1971).

All of these terms need to drop below a user-set tolerance. The tolerance for these cases is initially set to the same tolerance used for PWR cases. All cases except for two-0011-61 and 107161 - are able to converge to steady-state within the PWR convergence criteria when the droplet field is disabled. When the droplet field is enabled, two additional cases fail to converge to within the PWR tolerance-1071-55 and 4101-61. It is expected that CTF will not be able to converge as tightly for high-void cases, where there is a great deal of mass and energy transfer happening between the phases, as it does for single-phase cases. The fact that the rod conduction equation solution is explicitly coupled to the fluid energy solution, rather than an implicit coupling, likely worsens this problem.

The tolerance of the non-converging cases has to be increased to $1 \%$ for the energy balances, the mass balances, and the mass storage (Items 1, 2, 3, 4, and 5 from the list above). The tolerance for fluid energy storage has to be increased to $2 \%$. The tolerances used for PWR cases are $0.01 \%$ for Items $1,2,3$, and 4 and $0.5 \%$ for Items 5,6 , and 7 . Item 6 , the solid energy storage, has no impact on these simulations because the solids (heater rods, water rods, and fuel canister) are not modeled in $\mathrm{CTF}$ for these cases.

As a first step, CTF-predicted bundle average void at the outlet and thermal equilibrium quality is compared with the experimental results. Both values are area-weighted during the averaging process. The comparison of CTF-predicted and measured bundle average outlet void is shown in Figure 64 and the comparison of CTF-predicted and measured bundle average thermal equilibrium quality is shown in Figure 65.

There is a tendency for CTF to consistently overpredict the bundle-average void. The overprediction is more severe for the cases with lower amounts of void (about 40\%). At outlet void of about $80 \%$, the prediction almost matches the experimental values. If the same amount of energy is entering the CTF model as is entering the experimental facility, the mixture enthalpy at the outlet should be identical to the experiment (barring steam table differences), which means the void content should also be equal. It is possible the experimental facility loses heat along the axial length, 


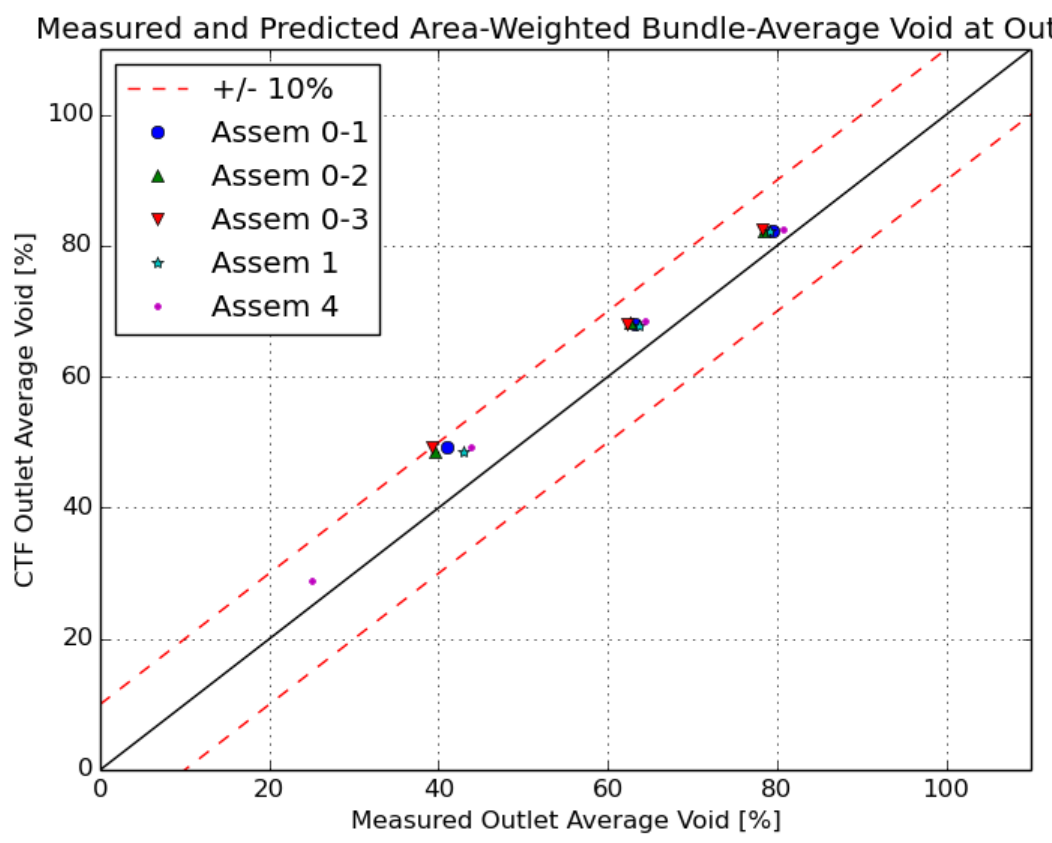

Figure 64. Comparison of measured and predicted bundle-averaged outlet void.

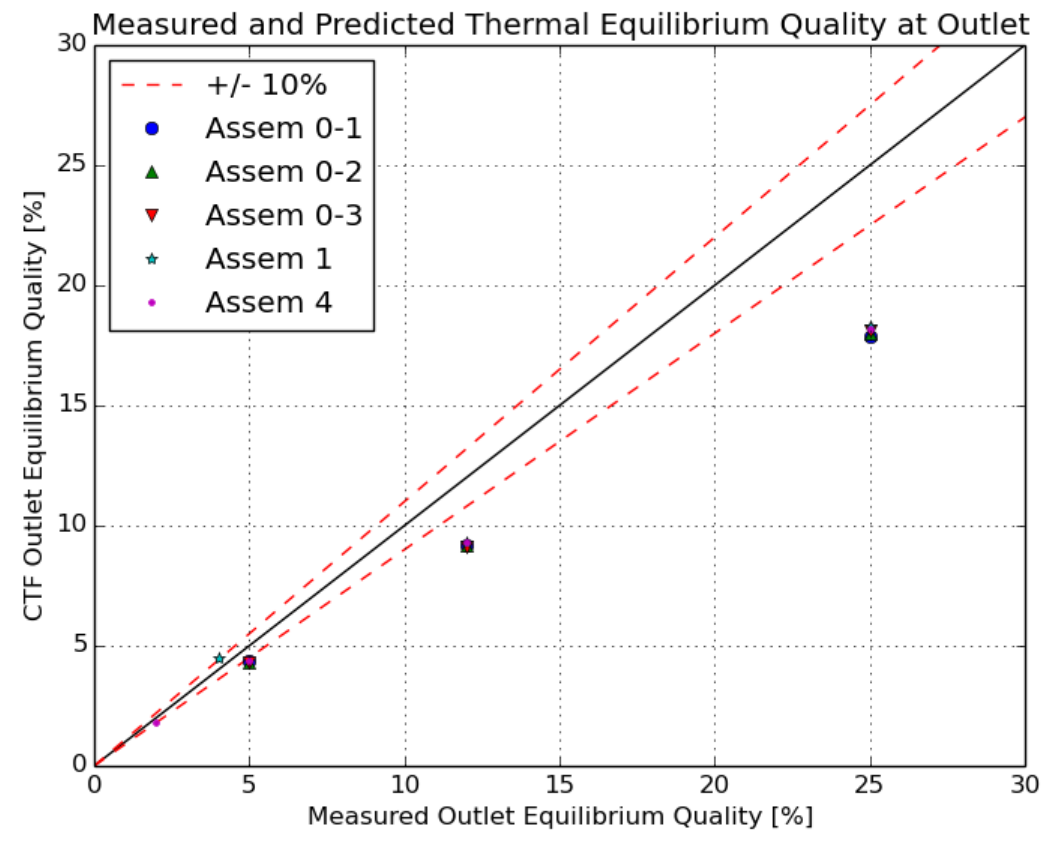

Figure 65. Comparison of measured and predicted bundle-averaged outlet thermal equilibrium quality. 
which therefore leads to a lower amount of void at the outlet compared with the perfectly adiabatic CTF simulation.

The behavior observed in Figure 65 is anomalous. Considering that the average outlet void predicted by CTF is consistently overestimated, one would expect that the exit thermal equilibrium quality would also be higher than experiment. The thermal equilibrium quality is a function of mixture enthalpy, which should be higher in the CTF model because of higher amounts of void. However, the comparison of predicted and measured quality indicates that CTF predicts substantially lower quality than the experiment at the outlet. The experiment specification is not clear on how exit flow and enthalpy were measured or how thermal equilibrium was calculated, so further investigation of this inconsistency is not possible.

Before comparing specific channel outlet void measurements to CTF predictions, it is useful to check the symmetry of the experimental data. To do this, the measurements are grouped into categories set by the channel types, which are

1. corner channel,

2. side channel,

3. normal inner channel, and

4. inner channel touching unheated element.

The grouping is not perfect, as none of the assembly types are eighth-core symmetric, and the channels touching unheated elements have different flow areas and wetted perimeters and touch different numbers of heated rods. Regardless, the selected categorization leads to a simpler organization of the data, and we should expect variation in corner and side channels due to assembly asymmetry to be minimal because of their large distance from the asymmetric center of the assembly. The check is done only for Assembly Types 0-1 and 0-2. Assembly Type 0-3 contains a large unheated section in the bundle that is asymmetric and close to the boundary rods, which we expect to lead to more significant asymmetry effects. Assembly Types 1 and 4 have non-uniform radial power distributions, which invalidate the selected data categorization.

Figures 66 and 67 give the mean void for each category as well as the spread of the data. The $\mathrm{x}$-axis contains the four categories. The $\mathrm{y}$-axis is the local subchannel void measurement. The dot gives the mean void for that category, and the error bars give the minimum and maximum void measurement for that category. The horizontal black lines give the bundle-average void at the outlet for each test in the figure. There are three tests shown in each figure (one figure per assembly type).

A few conclusions are drawn from the figures. First, the corner channel measurements tend to have the least scatter in measurement data, whereas the side channels tend to have the largest amount of scatter. Measurements for a given channel type tend to vary by as much as 10-20\%, which suggests that the measurement uncertainty may be larger than the $3 \%$ value quoted in the BFBT specification. Another observation is that the void drift phenomena becomes evident when comparing mean void to bundle-average void for all experiments. The corner and side voids are typically below the bundle-average value, whereas the inner channel void is typically above the bundle-average void. As expected, the channels that touch unheated elements often have the lowest void. The void in unheated channels is lowest for Assembly Type 0-2, which has two heater rods disabled in the center of the assembly. It is additionally noted that this trend is more exaggerated for test cases with lower total void content. As total void content increases, the outlet void distribution tends to be more uniform. Although conclusions on data symmetry cannot be drawn for the remaining assembly types, similar plots are shown in Figures 68-70 to demonstrate the void drift trend for the other assembly types.

Figure 71 gives an overview of the measured versus predicted subchannel void measurements for all tests. The data is grouped by assembly type, so multiple experiments are included in each dataset. The data shows that there is an overprediction of void; data is frequently over the $+10 \%$ line but never falls below the $-10 \%$ line. Also, the data appears to form into horizontal "stripes." This is due to the asymmetric behavior and spread of the experimental data compared with the symmetric behavior of the CTF predictions. The RMSE of all data is $7.95 \%$ compared with experimental data. 


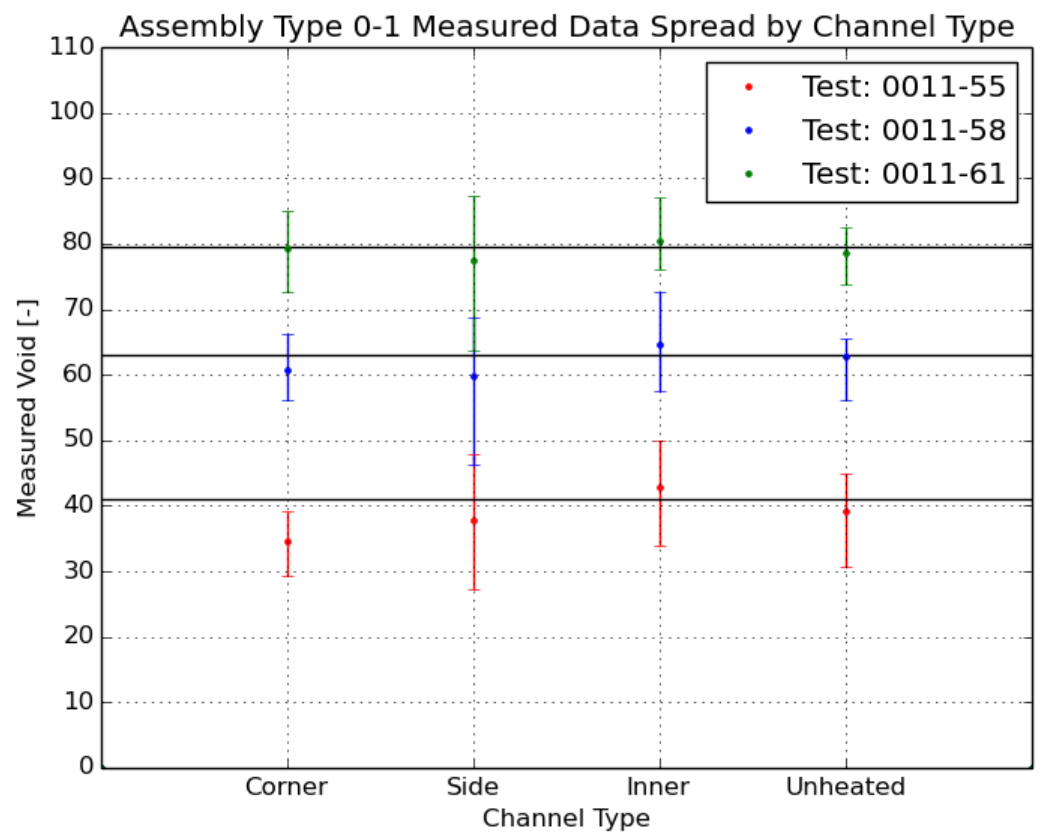

Figure 66. Summary of average void measurements for four channel categories in Assembly Type 0-1 experiments.

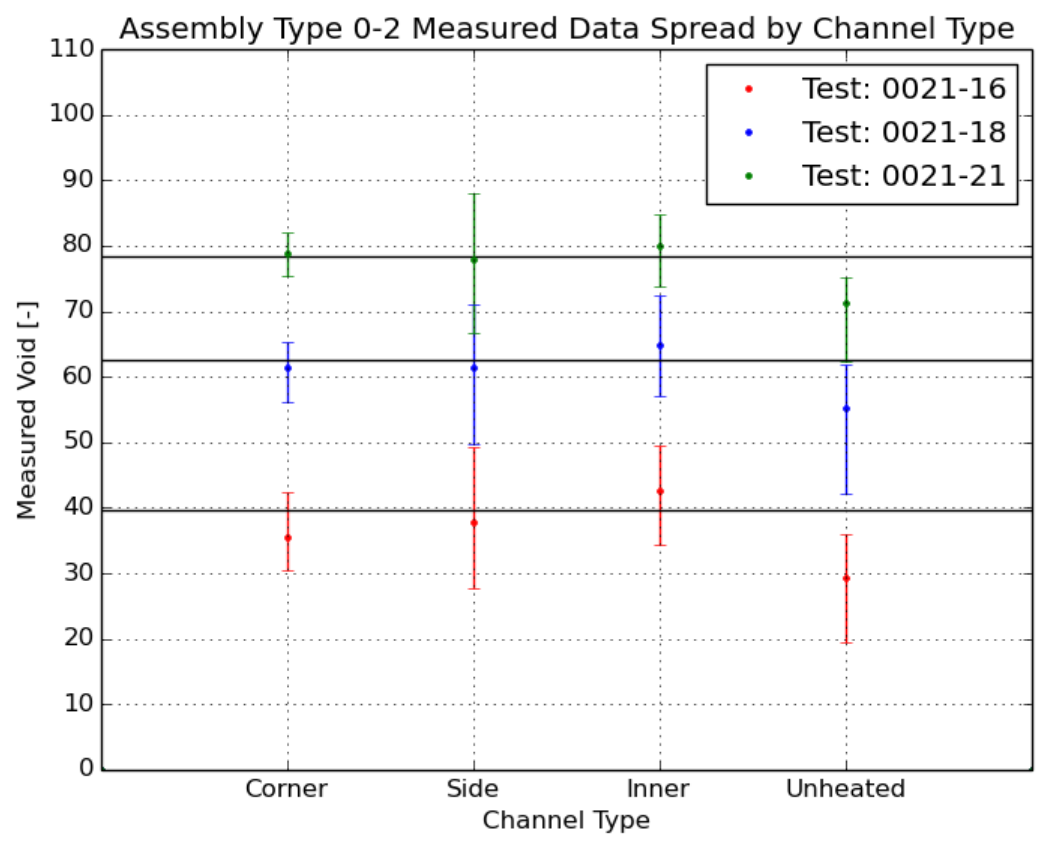

Figure 67. Summary of average void measurements for four channel categories in Assembly Type 0-2 experiments. 


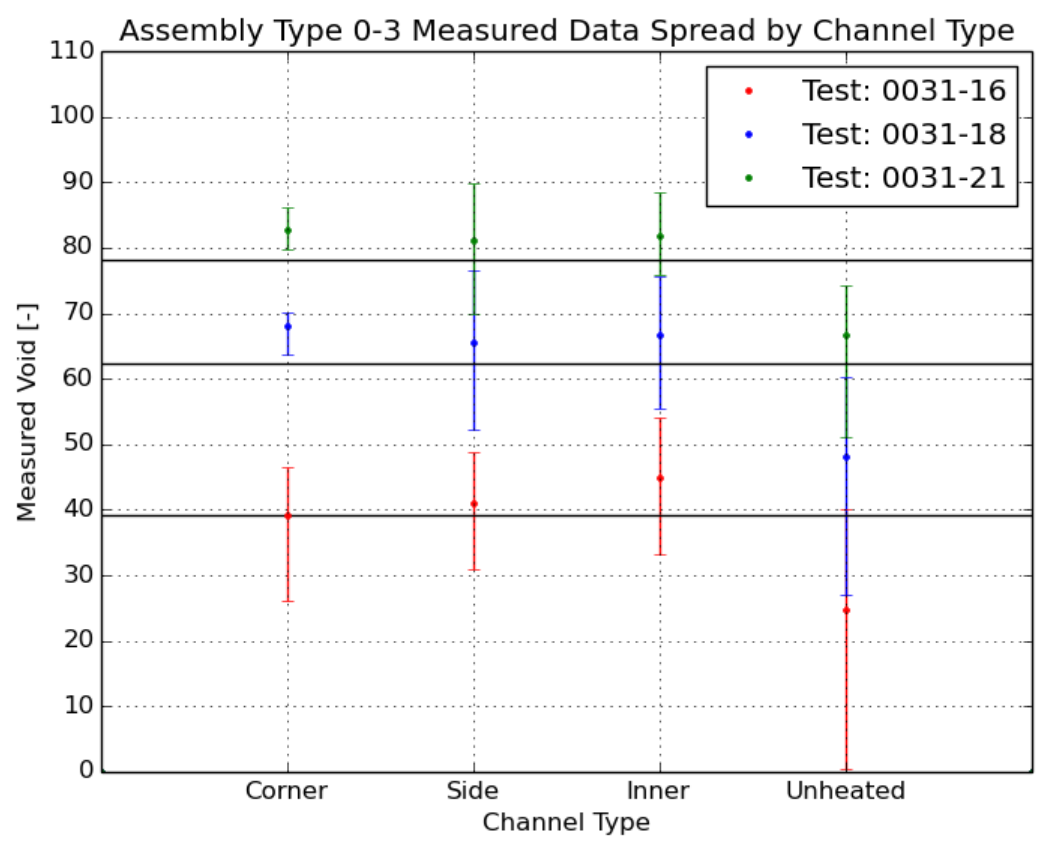

Figure 68. Summary of average void measurements for four channel categories in Assembly Type 0-3 experiments.

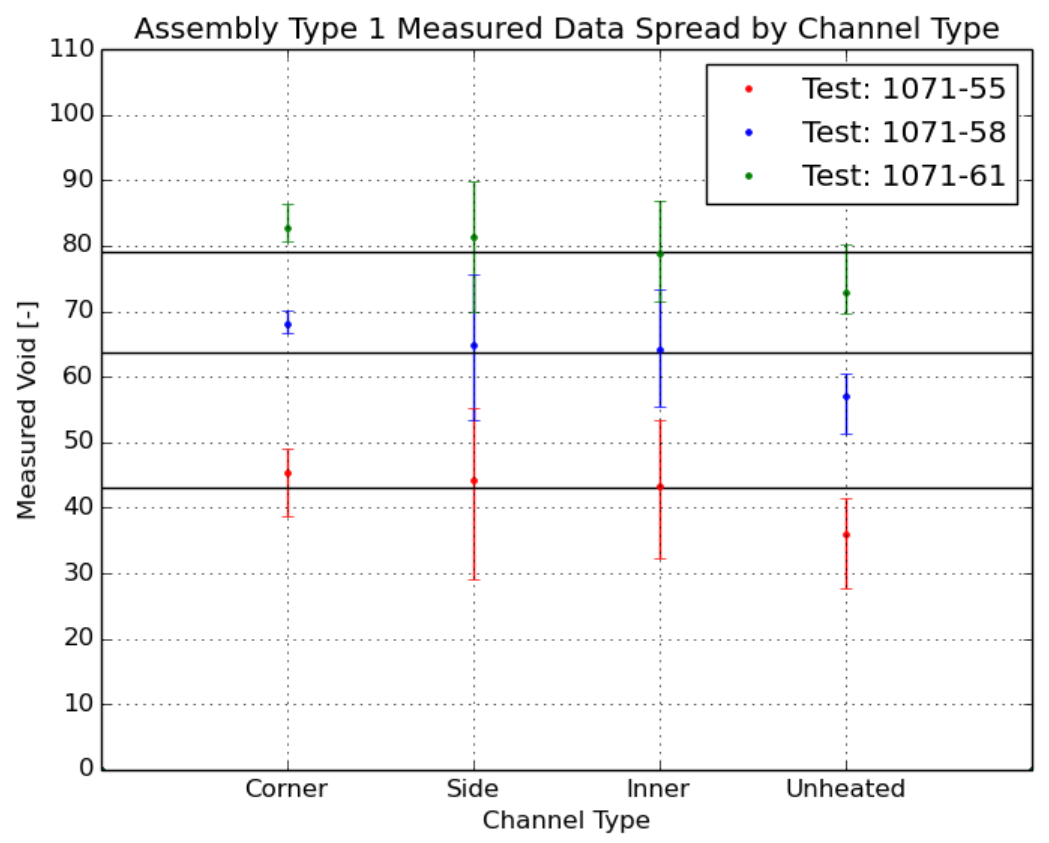

Figure 69. Summary of average void measurements for four channel categories in Assembly Type 1 experiments. 


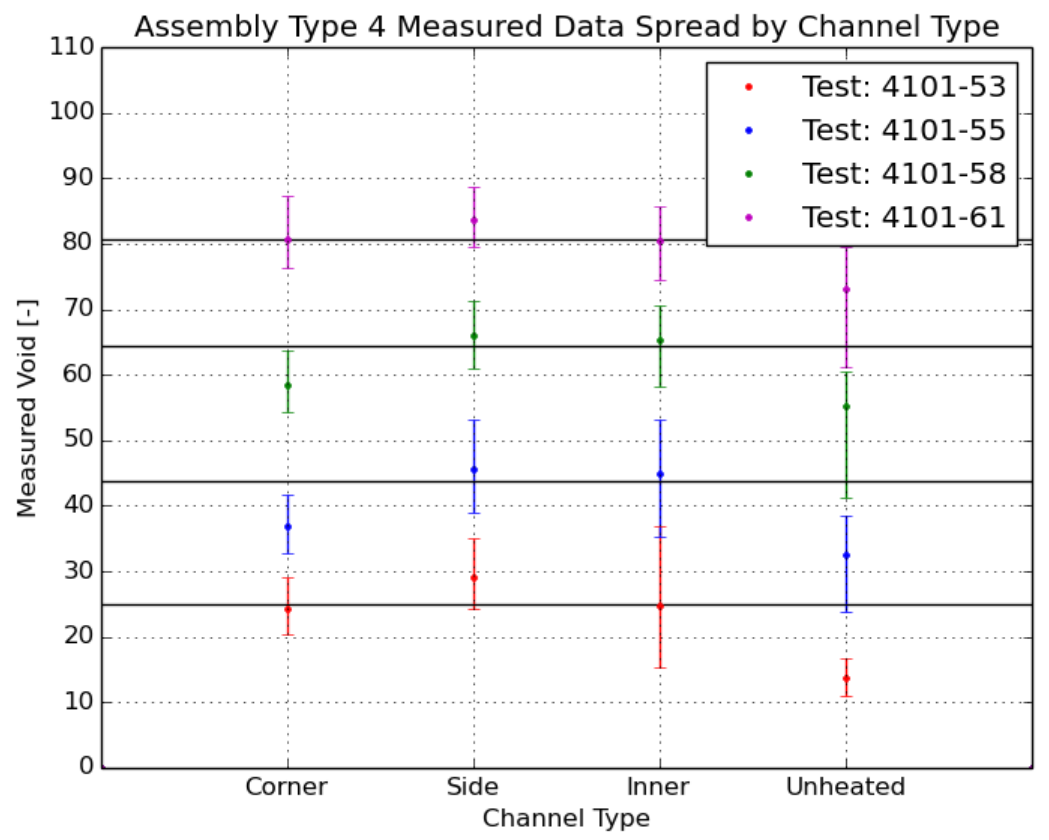

Figure 70. Summary of average void measurements for four channel categories in Assembly Type 4 experiments.

A second simulation of the cases is performed with the droplet field enabled to show the effect. Results for all tests are shown in Figure 72.

More insight is gained by breaking the data comparison into the four aforementioned channel categories. This is shown in Figures 73 (no droplets) and 74 (with droplets) for the corner type channels, Figures 75 (no droplets) and 76 (with droplets) for the side type channels, Figure 77 (no droplets) and 78 (with droplets) for the normal inner type channels, and Figure 79 (no droplets) and 80 (with droplets) for the inner channels touching unheated elements.

In every category, enabling the droplet field causes a degradation in prediction accuracy of a few percentage points. The worst predictions are for the channels that touch unheated elements. CTF tends to overpredict the void in these channels. RMSE for the unheated channels is $11.68 \%$. The inner channels, on the other hand, almost alway fall within the $\pm 10 \%$ bands in the plot. The RMSE for this channel type is only $5.52 \%$. As was observed in the GE $3 \times 3$ validation study, the error in the boundary channels tends to be higher. The corner channel RMSE is $7.61 \%$ and the side channel RMSE is $9.05 \%$. However, it was found in the GE $3 \times 3$ study that the corner channel exhibited the largest error compared with experimental data. The tendency for CTF to overpredict void near the unheated structures has been observed for other codes used to simulate this data in the OECD/NRC Benchmark [15].

It is useful to look into the effects of the CTF void drift model. The cases are rerun with the void drift model disabled (single- and two-phase turbulent mixing is still enabled and set to the previous values). The droplet model is disabled from this point forward. Figure 81 shows all measurement data compared with CTF predictions when the void drift model is disabled. As shown in the figure, the RMSE increases by about 1 percentage point. Figures 82-85 show the results broken down by channel category when the void drift model is disabled. The results show that the corner type channel witnesses the largest increase in error, from $7.61 \%$ to $11.56 \%$. The side channel also sees an increase in error, from $9.05 \%$ to $11.82 \%$. The channels touching an unheated element experience practically no change in error, from $11.68 \%$ to $11.56 \%$ and the inner type channels actually see a slight improvement in error, dropping from $5.52 \%$ to $4.62 \%$. Results for the four different groupings and three different parametric studies are shown in Table 5 . 


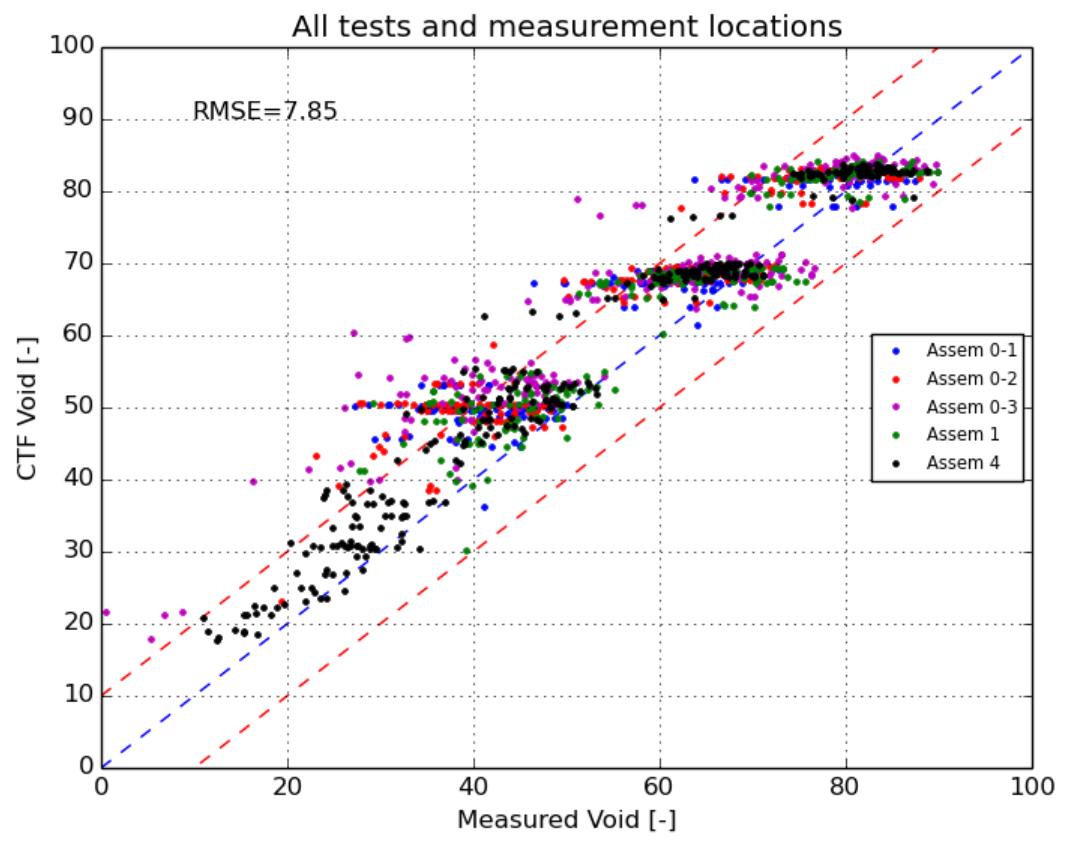

Figure 71. Comparison of CTF predictions and measurements of subchannel void for all BFBT void distribution tests.

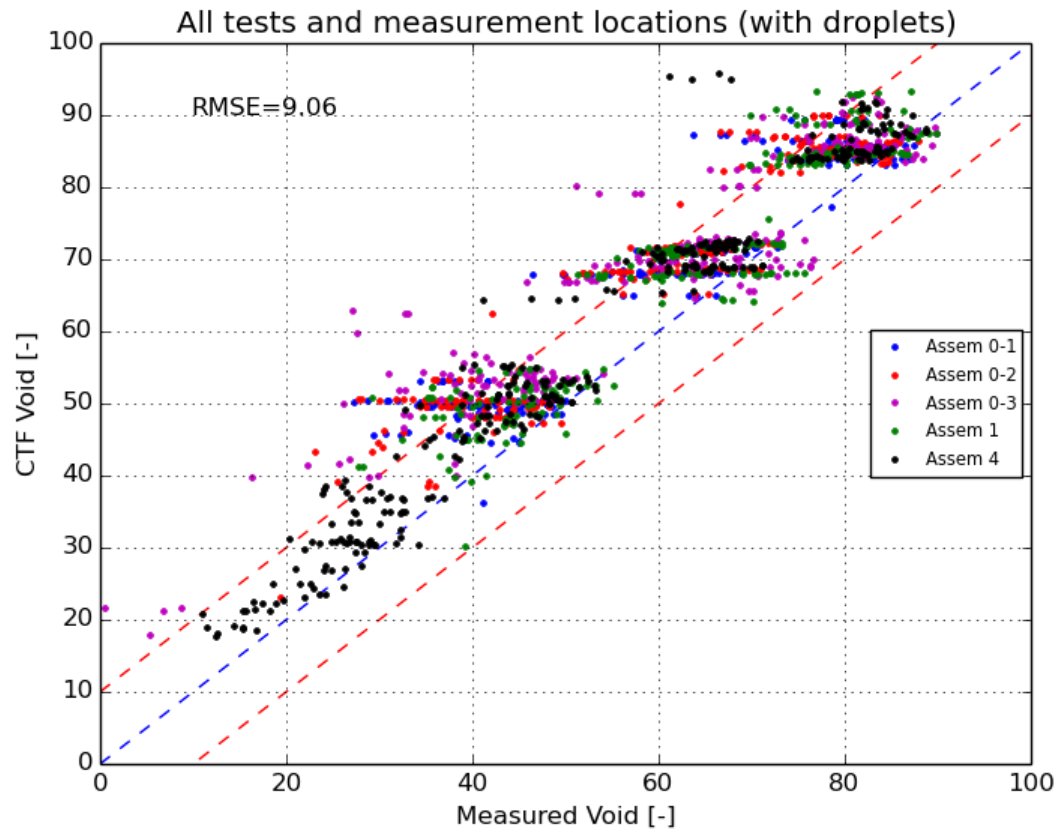

Figure 72. Comparison of CTF predictions and measurements of subchannel void for all BFBT void distribution tests (with droplets). 


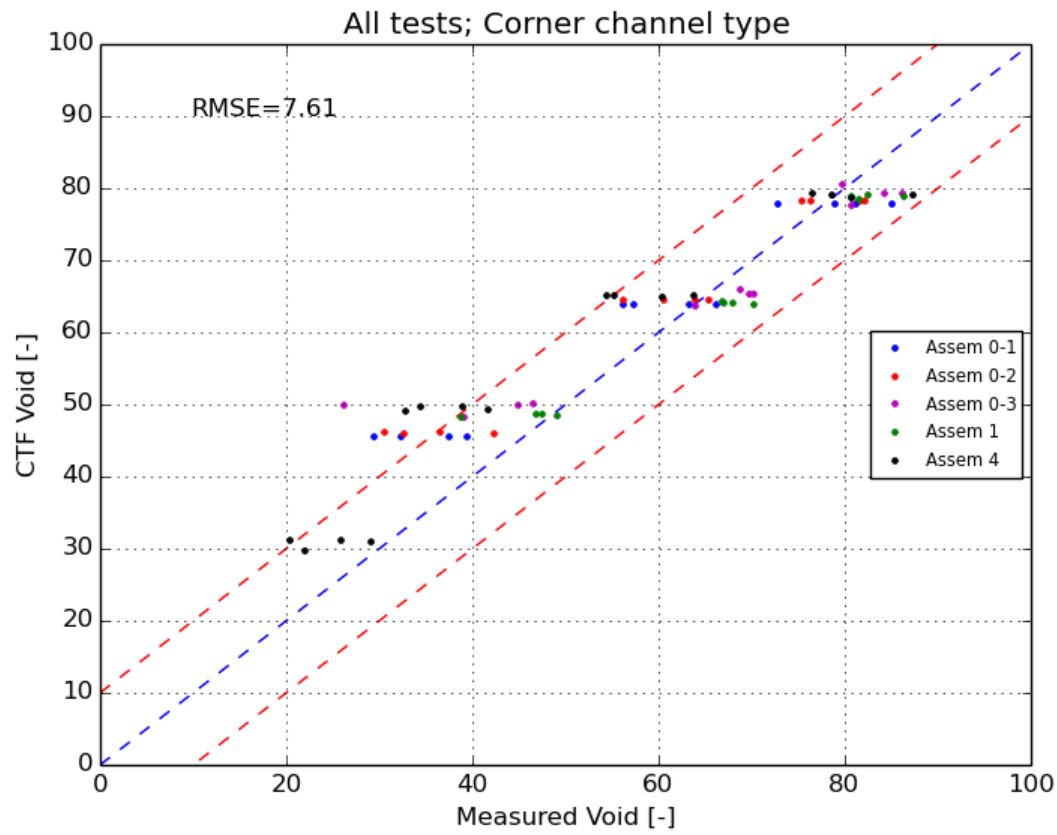

Figure 73. Comparison of CTF predictions and measurements of subchannel void for corner-type channels in all modeled BFBT void distribution tests.

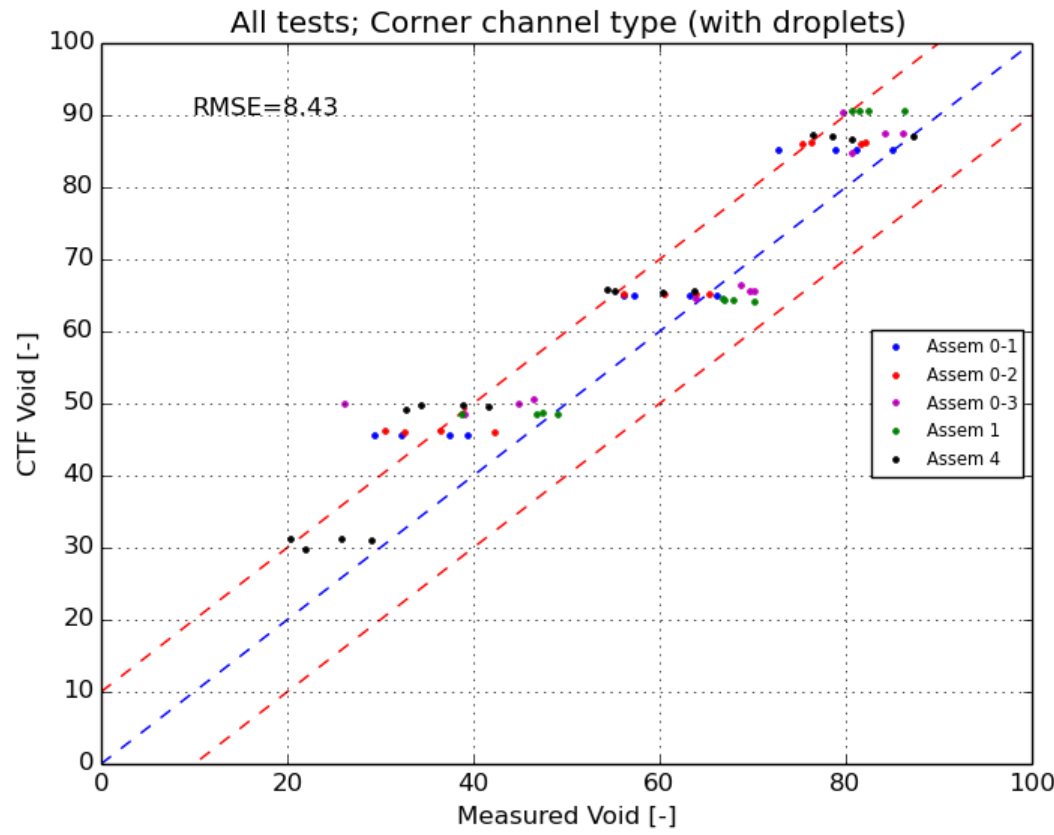

Figure 74. Comparison of CTF predictions and measurements of subchannel void for corner-type channels in all modeled BFBT void distribution tests (with droplets). 


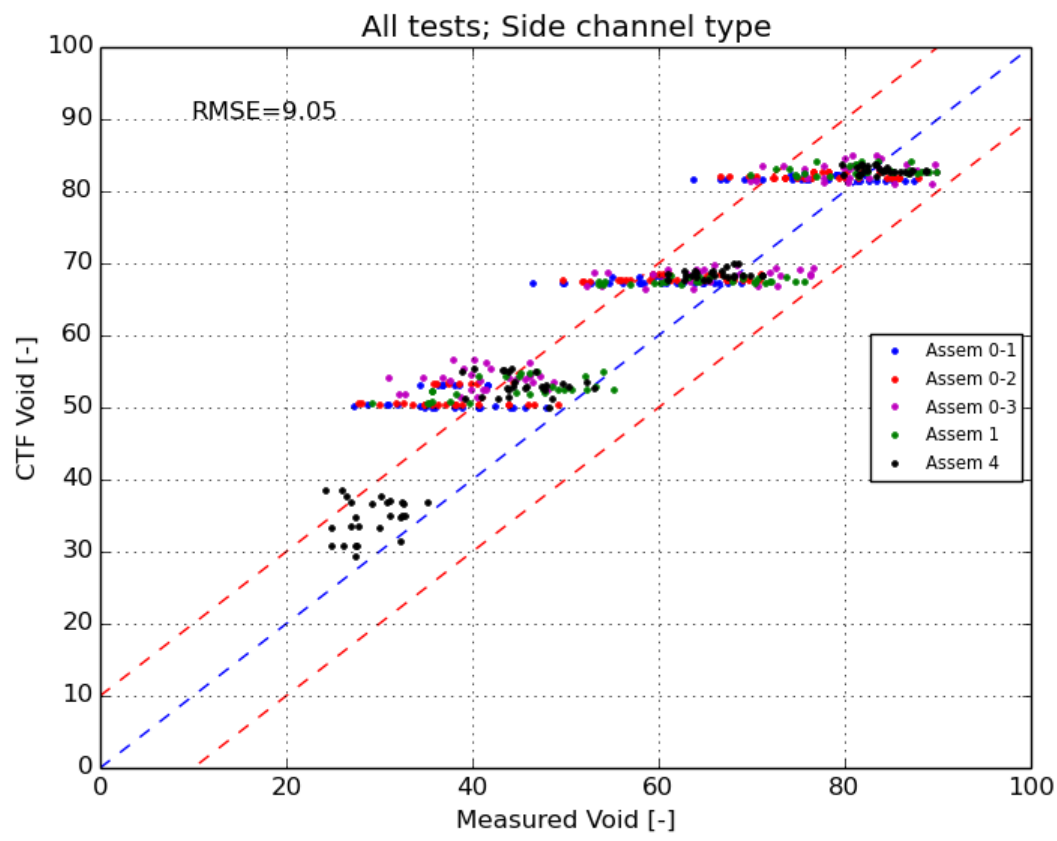

Figure 75. Comparison of CTF predictions and measurements of subchannel void for side-type channels in all modeled BFBT void distribution tests.

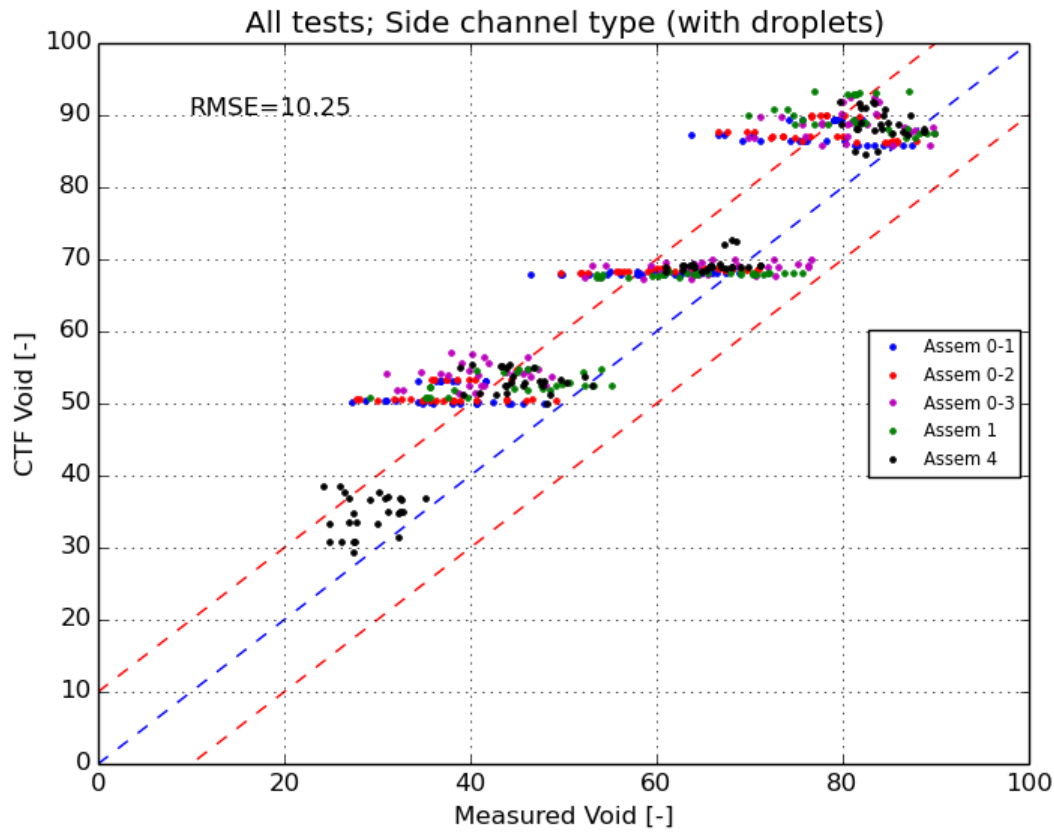

Figure 76. Comparison of CTF predictions and measurements of subchannel void for side-type channels in all modeled BFBT void distribution tests (with droplets). 


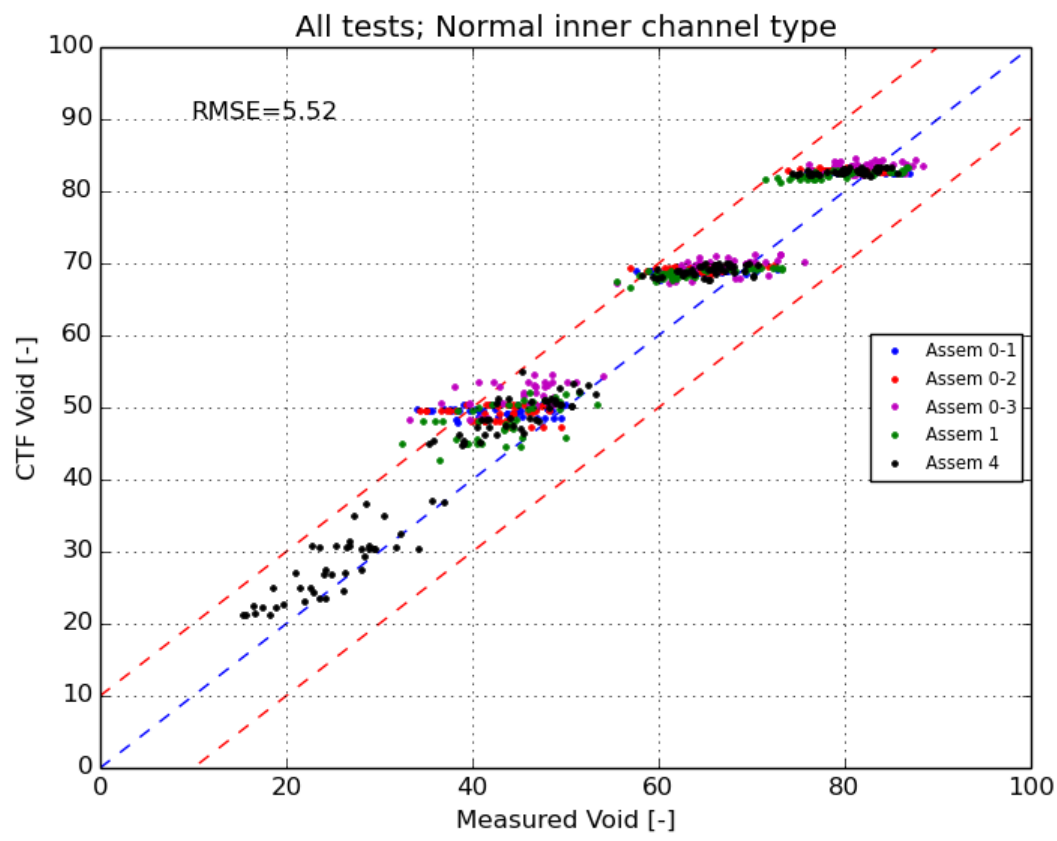

Figure 77. Comparison of CTF predictions and measurements of subchannel void for inner-type channels in all modeled BFBT void distribution tests.

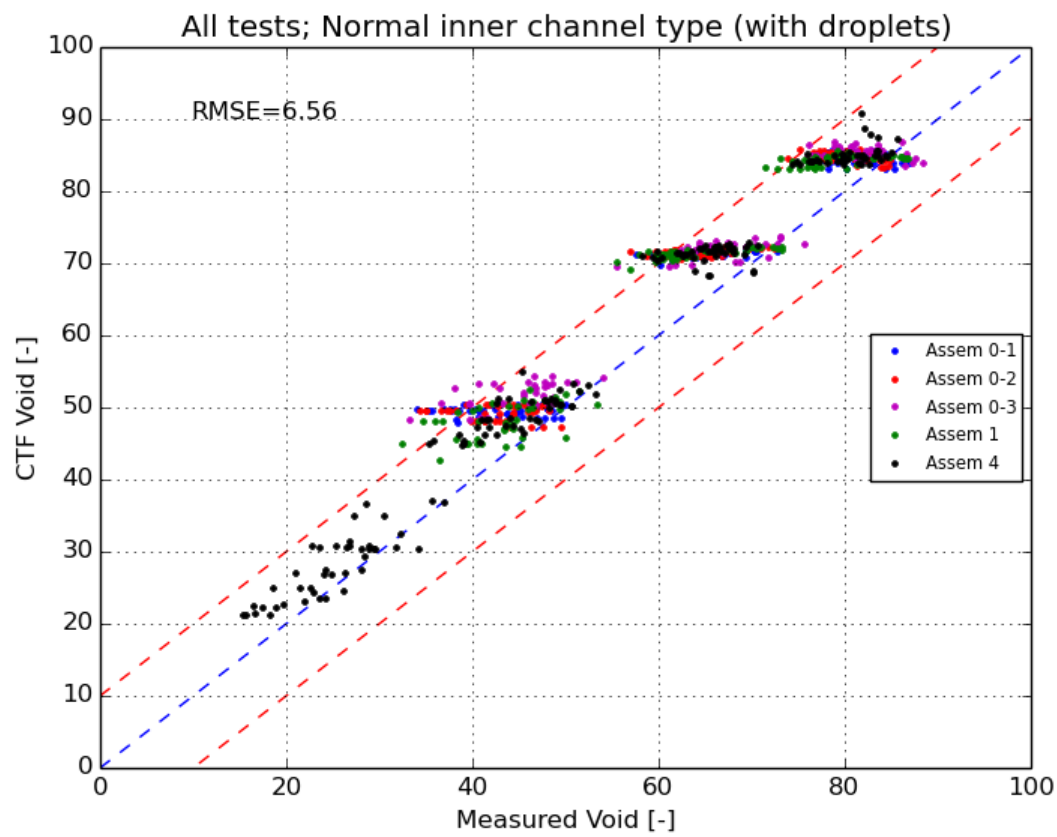

Figure 78. Comparison of CTF predictions and measurements of subchannel void for inner-type channels in all modeled BFBT void distribution tests (with droplets). 


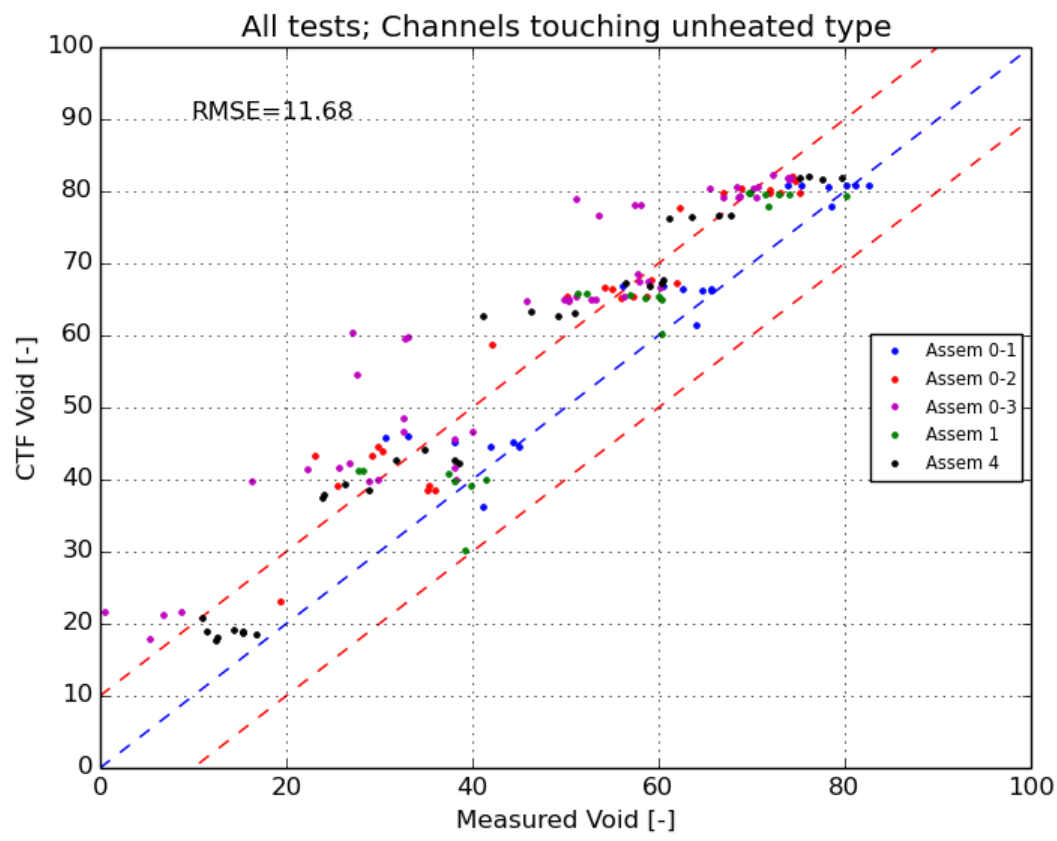

Figure 79. Comparison of CTF predictions and measurements of subchannel void for unheated-type channels in all modeled BFBT void distribution tests.

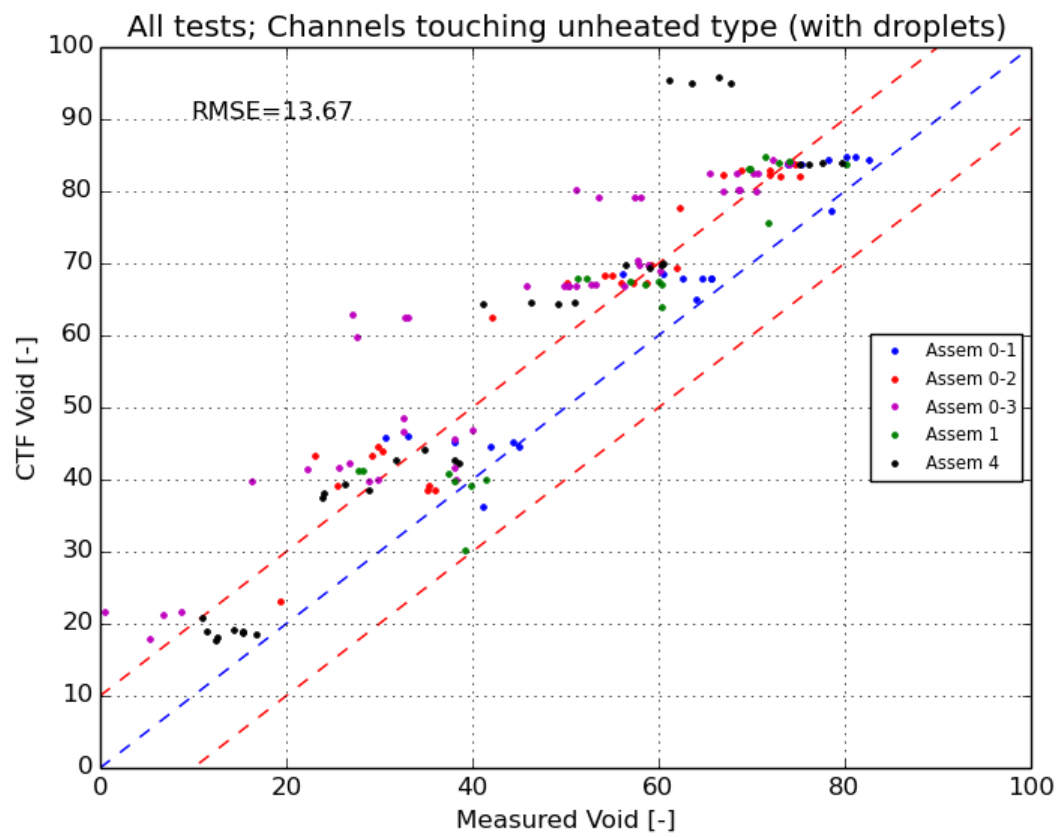

Figure 80. Comparison of CTF predictions and measurements of subchannel void for unheated-type channels in all modeled BFBT void distribution tests (with droplets). 


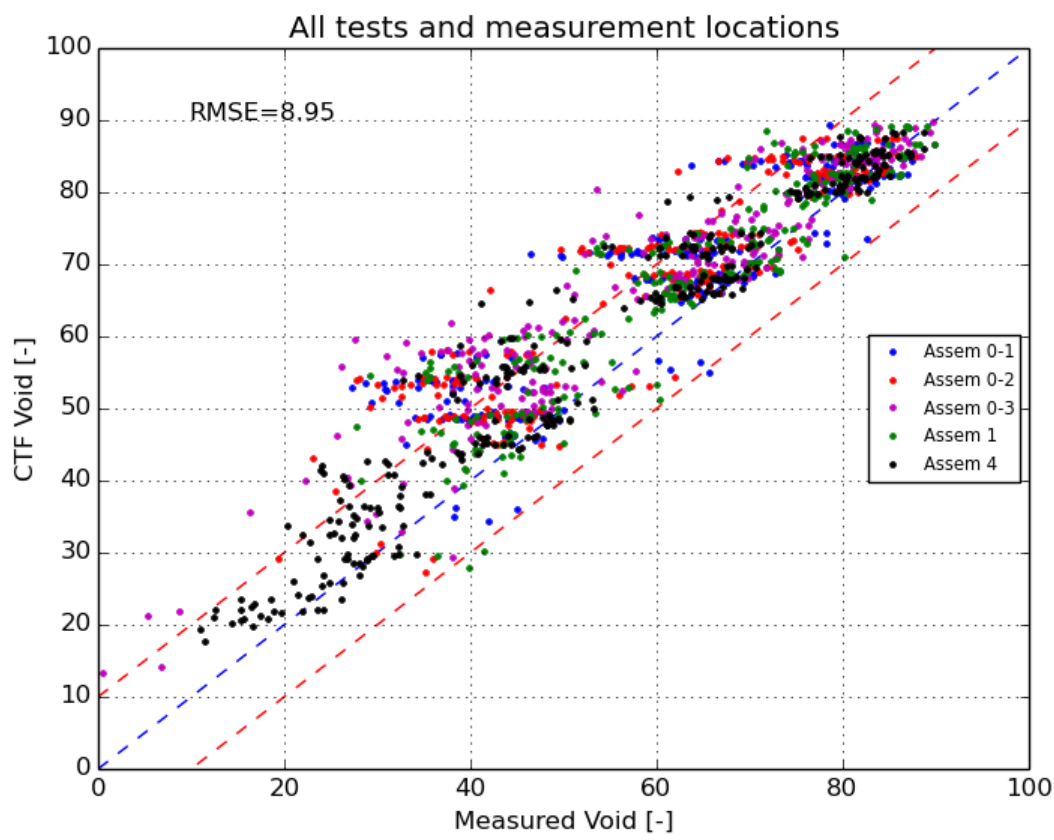

Figure 81. Comparison of CTF predictions and measurements of subchannel void for all channels in all modeled BFBT void distribution tests when void drift model is disabled.

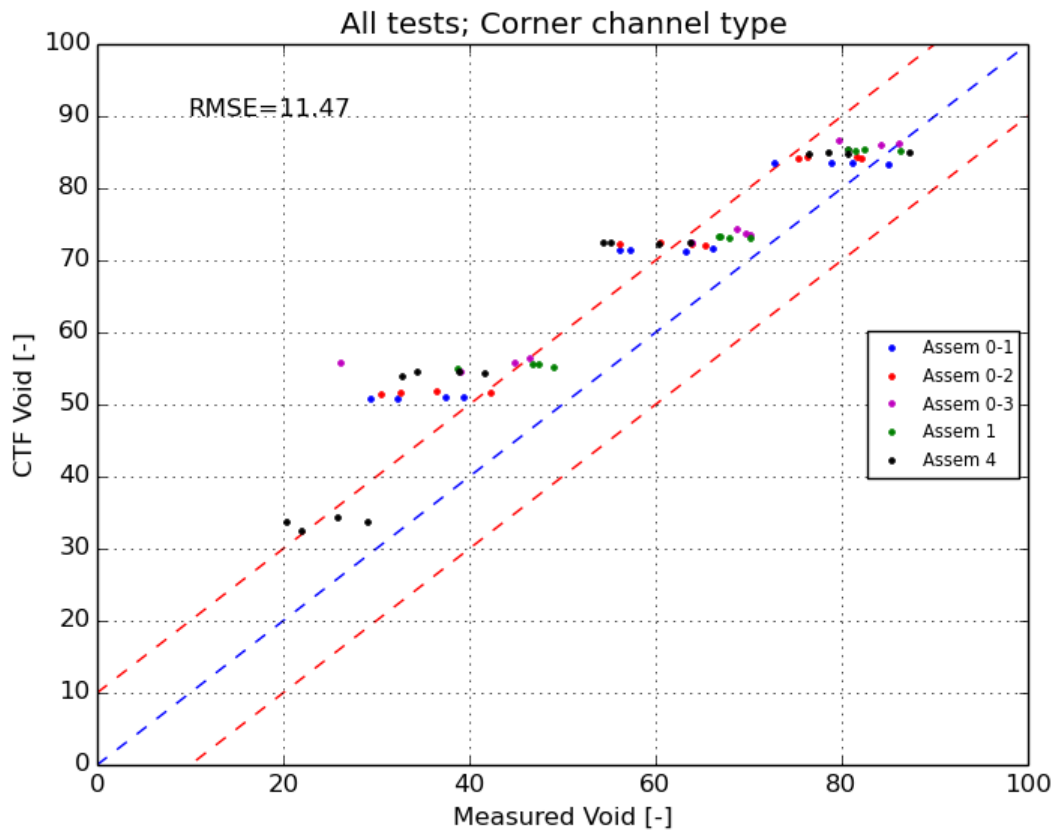

Figure 82. Comparison of CTF predictions and measurements of subchannel void for corner channels in all modeled BFBT void distribution tests when void drift model is disabled. 


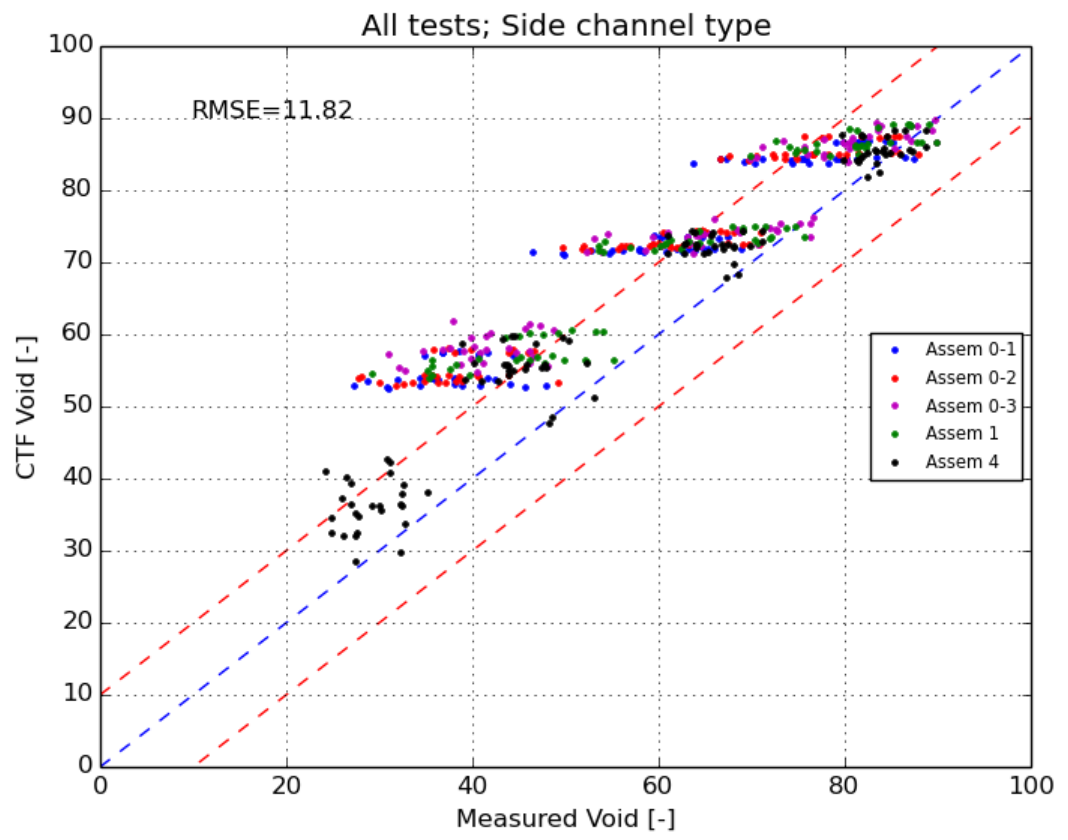

Figure 83. Comparison of CTF predictions and measurements of subchannel void for side channels in all modeled BFBT void distribution tests when void drift model is disabled.

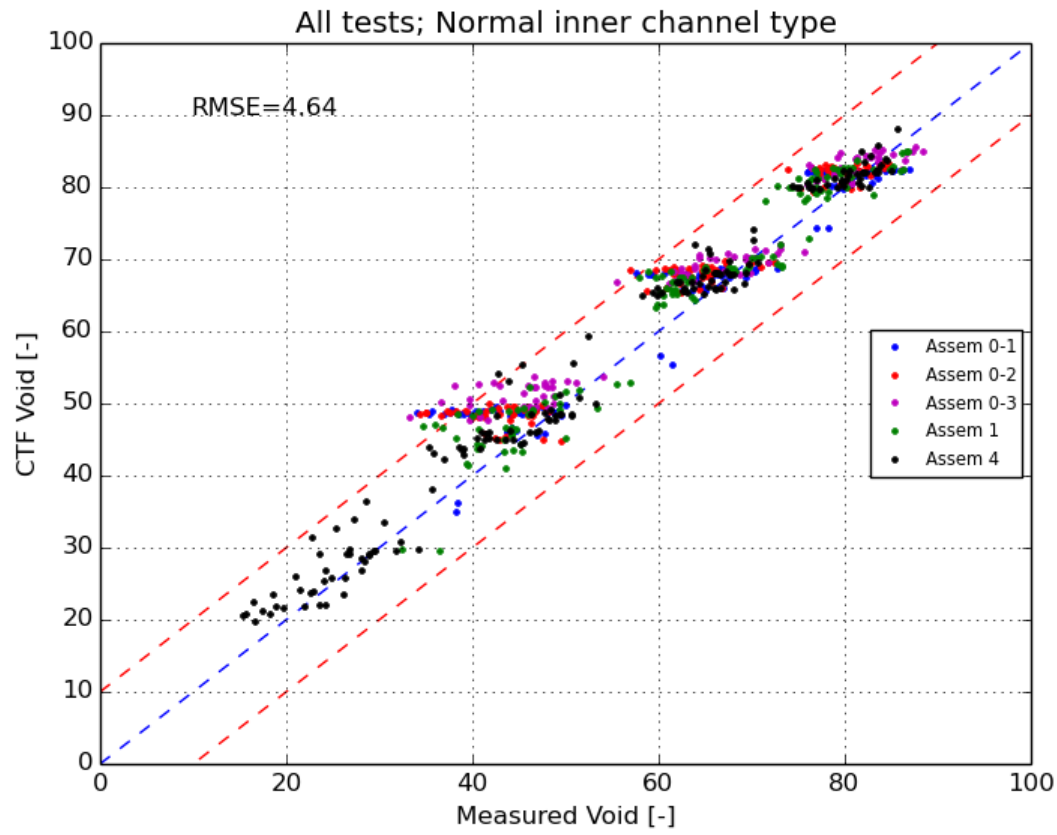

Figure 84. Comparison of CTF predictions and measurements of subchannel void for inner channels in all modeled BFBT void distribution tests when void drift model is disabled. 


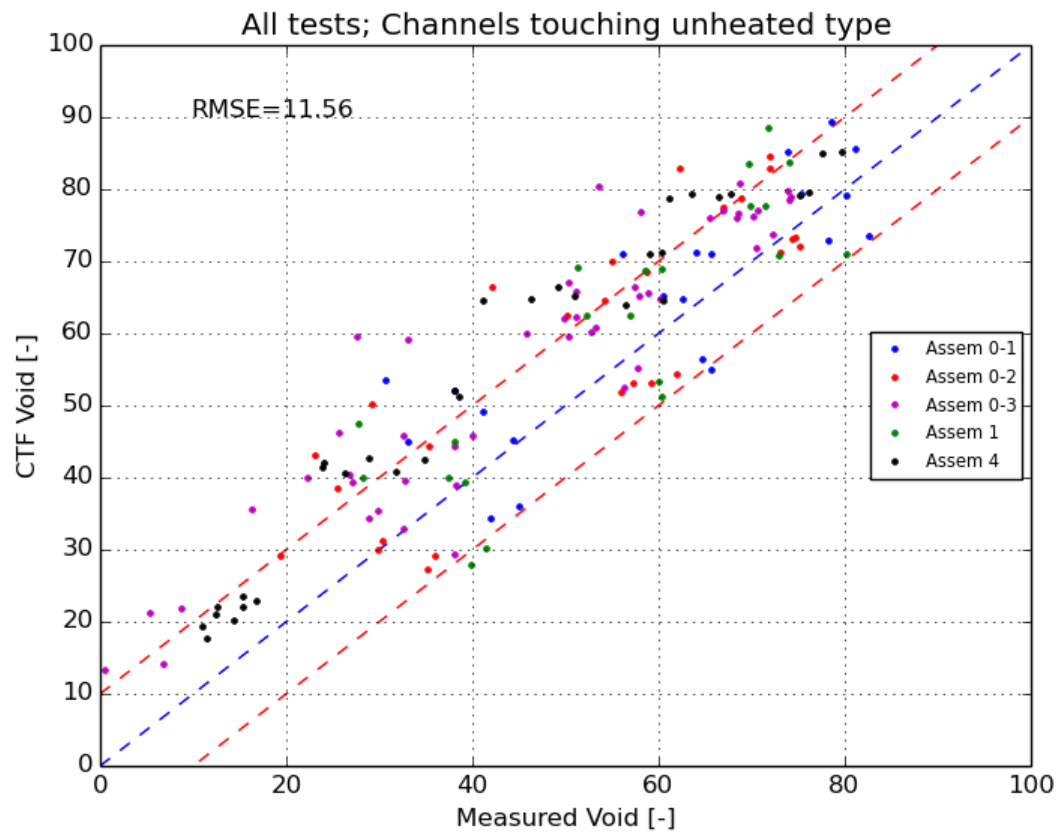

Figure 85. Comparison of CTF predictions and measurements of subchannel void for unheated channels in all modeled BFBT void distribution tests when void drift model is disabled.

Table 5. Summary of the CTF predictions of BFBT void distribution cases

\begin{tabular}{lrrr}
\hline Channel & $\begin{array}{r}\text { No Drops } \\
\text { Group }\end{array}$ & $\begin{array}{r}\text { With Drops } \\
\text { With Void Drift }\end{array}$ & $\begin{array}{r}\text { No Drops } \\
\text { With Void Drift }\end{array}$ \\
\hline Corner & 7.61 & 8.43 & 11.47 \\
Side & 9.05 & 10.25 & 11.82 \\
Inner & 5.52 & 6.56 & 4.64 \\
Unheated & 11.68 & 13.67 & 11.56 \\
\hline All & 7.85 & 9.06 & 8.95 \\
\hline
\end{tabular}


As it has been shown that there is a tendency for CTF to globally over-predict measured void data, it is important to use the data to look at trends. In other words, the void drift phenomena causes void to migrate from smaller area channels to higher area channels. It is important to look for this behavior in the simulations. The void drift model should be enabled and disabled to observe the impact of the model on results. To do this, void data is visualized over the diagonals of each assembly. Figure 86 gives an example of the two diagonal lines that are struck through the assemblies. The example is given for Assembly Type 0-1, but the process is the same for all five assembly types. Note that the red line goes from the northwest to the southeast, and the blue line goes from the southwest to the northeast. The experimental data along these lines will be shown with dots, and the CTF predictions will be shown with lines. The red dots and lines will be for the northwest to southeast diagonal, and the blue dots and lines will be for the southwest to northwest diagonal.

Figure 87 shows results for Test 0011-55, which is from Assembly Type 0-1. Figure 88 shows the same results, but with the void drift model disabled in the CTF simulation. In both cases, the CTF results are fairly symmetric. The red line has slightly different behavior because the center of the bundle has two guide tubes that lead to an asymmetric rod placement. In both cases, CTF captures the reduction in void in the center of the model because of the presence of the unheated guide tubes. However, the reduction is not as severe as it is in the experimental results.

Additionally, the experimental results see a jump up in void for the center channel. This behavior is likely due to the design of the spacer grid, which offers no flow resistance to the central subchannel. Despite the central subchannel having a smaller hydraulic diameter than surrounding channels (and thus a higher frictional pressure drop), the lack of a form loss allows the velocity to climb higher than its neighbors, which causes the higher velocity void to seek the path of least flow resistance and migrate into the center subchannel.

With void drift disabled, CTF sees a larger drop in void in the center channel than when void drift is enabled. Void increasing in the center subchannel is the correct behavior due to the lack of a form loss in that channel. The CTF void drift model acts to move void from lower to higher velocity subchannels, so turning the void drift model on causes void to migrate from the larger area channels (which have lower velocity) into the center channel (which has higher velocity).

When void drift is enabled, the trend of void decreasing in the corner channels is predicted. When void drift is disabled, the void is highest in the corner channels, which is incorrect. While the trend is predicted correctly, the magnitude of the drop is less in CTF than in the experimental results. It is possible that the experimental facility walls are not perfectly adiabatic, which may explain the over-prediction of void in CTF. Overall, there is an improvement of about 1 percentage point for the RMSE of both lines of CTF predictions when compared to the experimental data.

As has been previously noted, the void drift effect becomes less severe at higher overall bundle void content. Test 0011-61 is also run in Assembly Type 0-1; however, it is run at a much higher power, leading to a bundle average void that is about double that of Test 0011-55. Figure 89 shows the void distribution on the diagonals with void drift enabled. Figure 90 shows the same data, but with void drift disabled in the CTF simulations.

It is observed that the CTF results, with void drift enabled, compare very nicely to experimental data because the void drift phenomena is not as severe. The RMSE is between 4 and 6 percentage points lower than the lower void case, Test 0011-55. Disabling the void drift model actually leads to an improvement in one of the diagonals when comparing to experimental data due to the large asymmetry in the corner results.

The low-void test from Assembly 0-2, which has four unheated rods in the bundle center, is shown in Figure 91 with void drift enabled and in Figure 92 with void drift disabled. Similar trends are observed, but it is noted that void content decreases more in the center than it did in Assembly 0-1 due to the disabling of the two center heater rods. As in the case of Assembly Type 0-1, the void drift effect is less prominent in the higher void case, which is shown in Figure 93 (with void drift) and Figure 94 (without void drift). Note, though, that enabling the void drift model in CTF allows a great deal of void to migrate into the center of the bundle, which is unrealistic compared with the experimental results.

Test case 0031-16 is run in Assembly Type 0-3, which has seven unheated rods and two guide tubes in a position that is offset from the center of the bundle. This leads to an asymmetric 


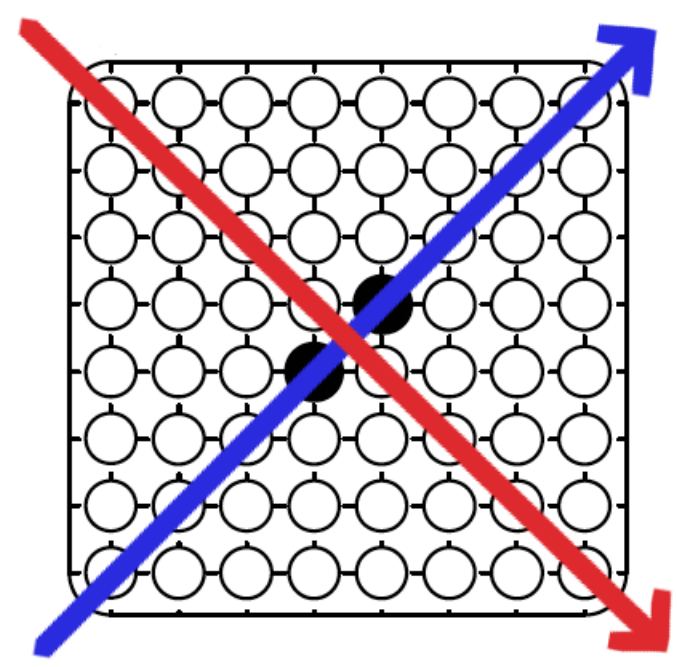

Figure 86. Example of diagonal lines from where subchannel void data is extracted.

distribution in the void. Figure 95 shows the CTF results with void drift on, and Figure 96 shows the results with void drift disabled. The red line is shifted to the right because of the additional unheated rods in the southeast quadrant of the assembly. This trend matches the data (the red dots also shift to the right). However, lowest void appears in the channel at Index 6 in the plots in the experimental data. CTF predicts the lowest void at Index 5 (center of the bundle). This is the case whether void drift is enabled or disabled, so the turbulent mixing must be driving void out of the center channel due to the higher velocity in the center channel. Because of the better-predicted void in the unheated region with void drift disabled, the RMSE is slightly better when void drift is disabled. A similar result is found for the higher-void case of Assembly 0-3; the void drift model gets the corner channels right but causes too much void to drift into the unheated region of the bundle. Figures 97 and 98 show this behavior.

Tests from Assembly Type 1 have a non-uniform axial and radial power distribution. The assembly has two guide tubes in the center like Assembly Type 0-1. The low-void case shows slightly better agreement with the data with void drift enabled (Figure 99) compared with void drift disabled (Figure 100). As with other assembly types, the higher-void case comparison is slightly worse with void drift enabled. Figures 101 and 102 show the results for the high void case with and without void drift, respectively.

Finally, Assembly Type 4 contains a non-uniform radial power distribution, uniform axial power distribution, and one large water rod in the center of the model. Therefore, there is no center channel measurement or CTF prediction for these cases. The low-void case is shown in Figure 103 with void drift and Figure 104 without void drift. High-void case results are shown in Figure 105 with void drift and Figure 106 without void drift. Improvements in RMSE are more significant for the lower-void cases, but in general, data compares more favorably for this assembly type than for any of the others that are tested in this study. 


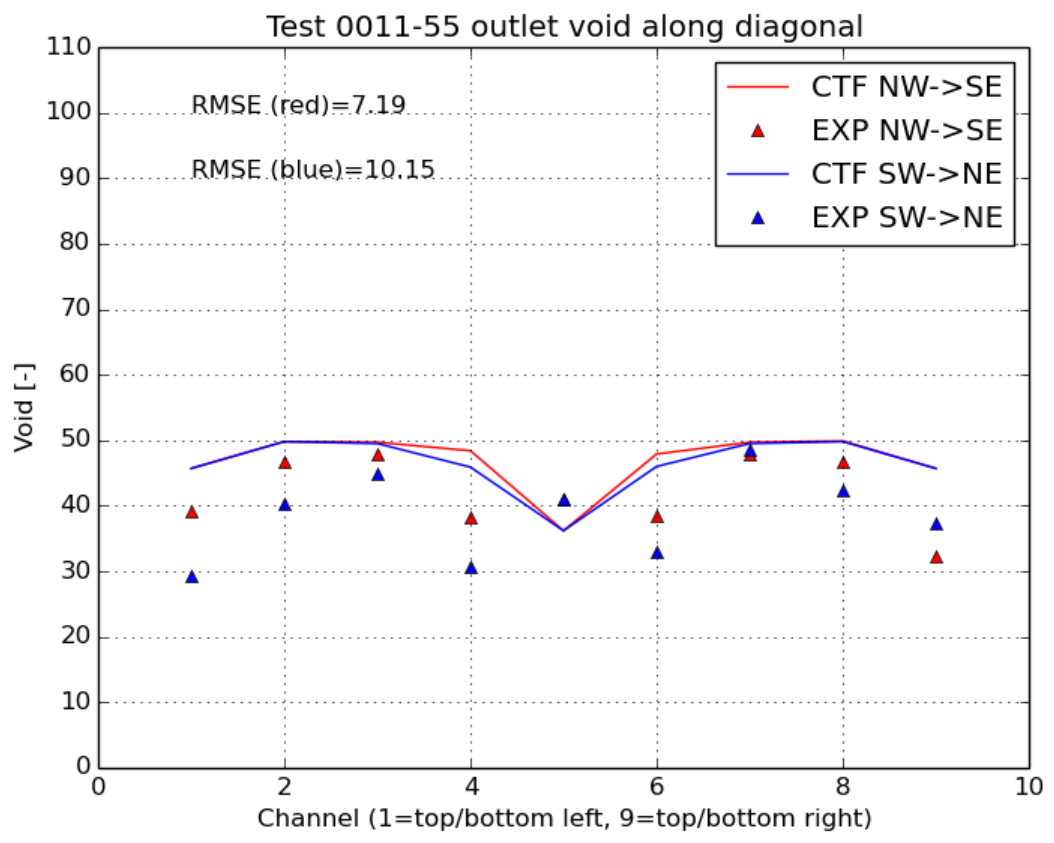

Figure 87. Measured and predicted void along diagonals of Test 0011-55.

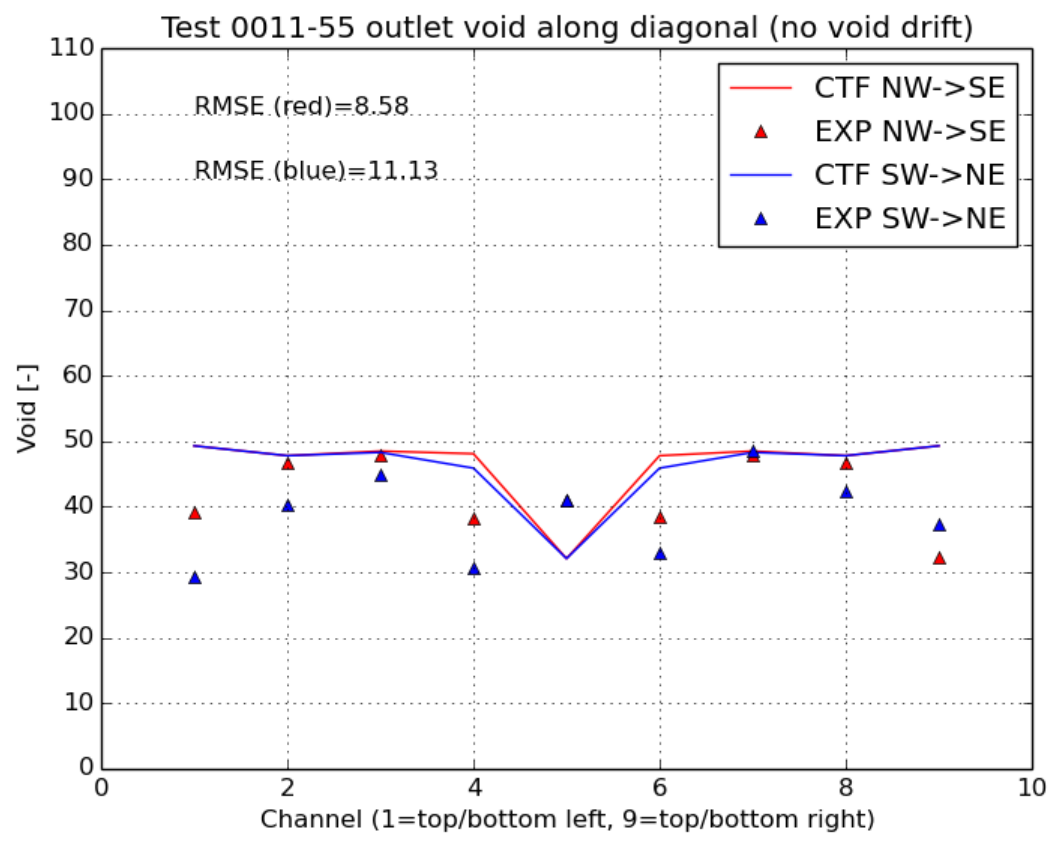

Figure 88. Measured and predicted void along diagonals of Test 0011-55 with void drift disabled. 


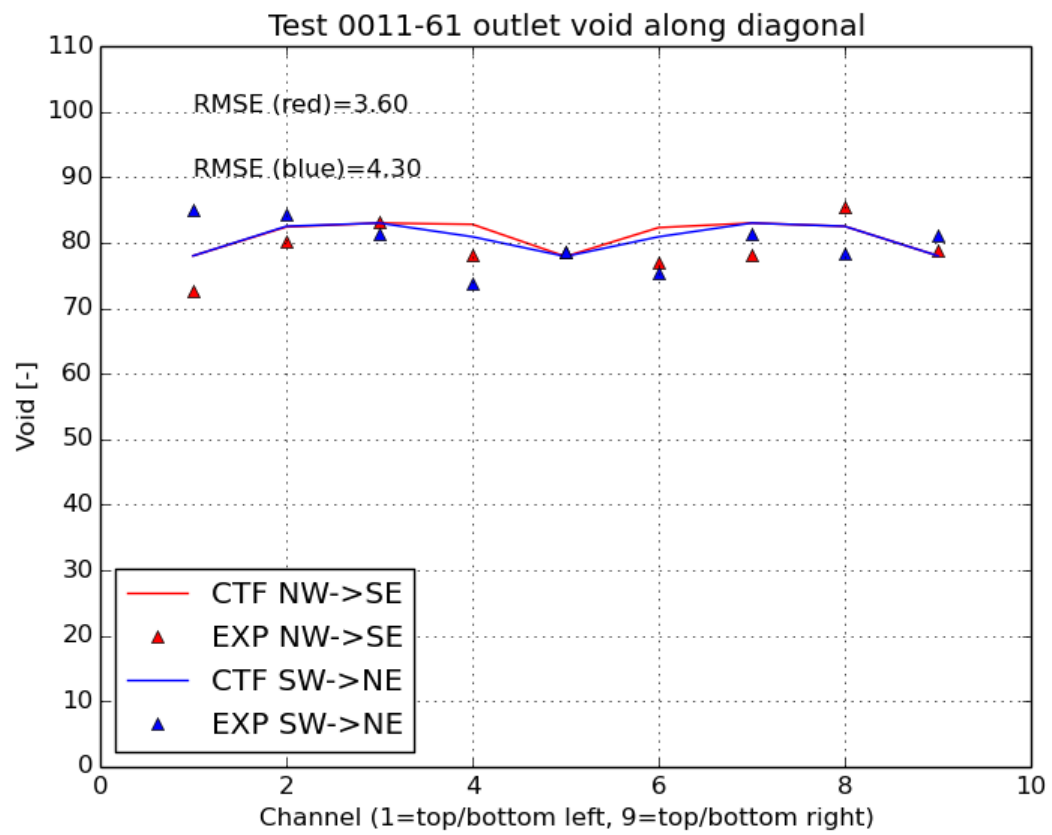

Figure 89. Measured and predicted void along diagonals of Test 0011-61.

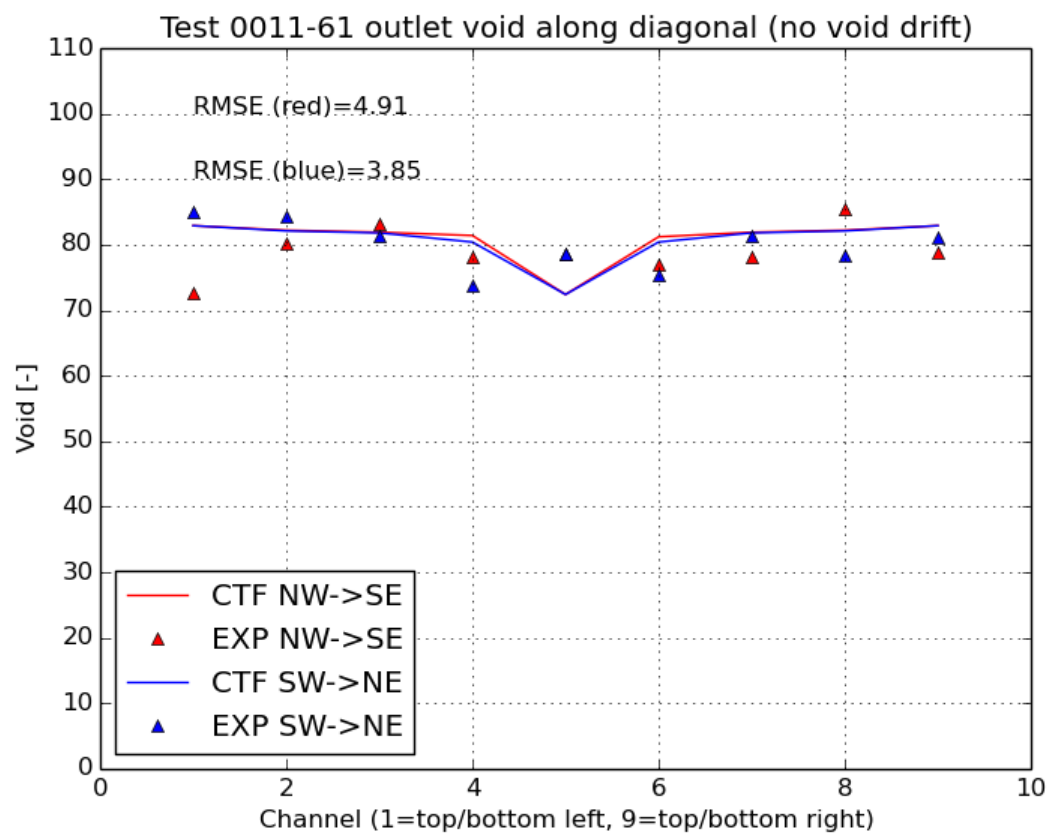

Figure 90. Measured and predicted void along diagonals of Test 0011-61 with void drift disabled. 


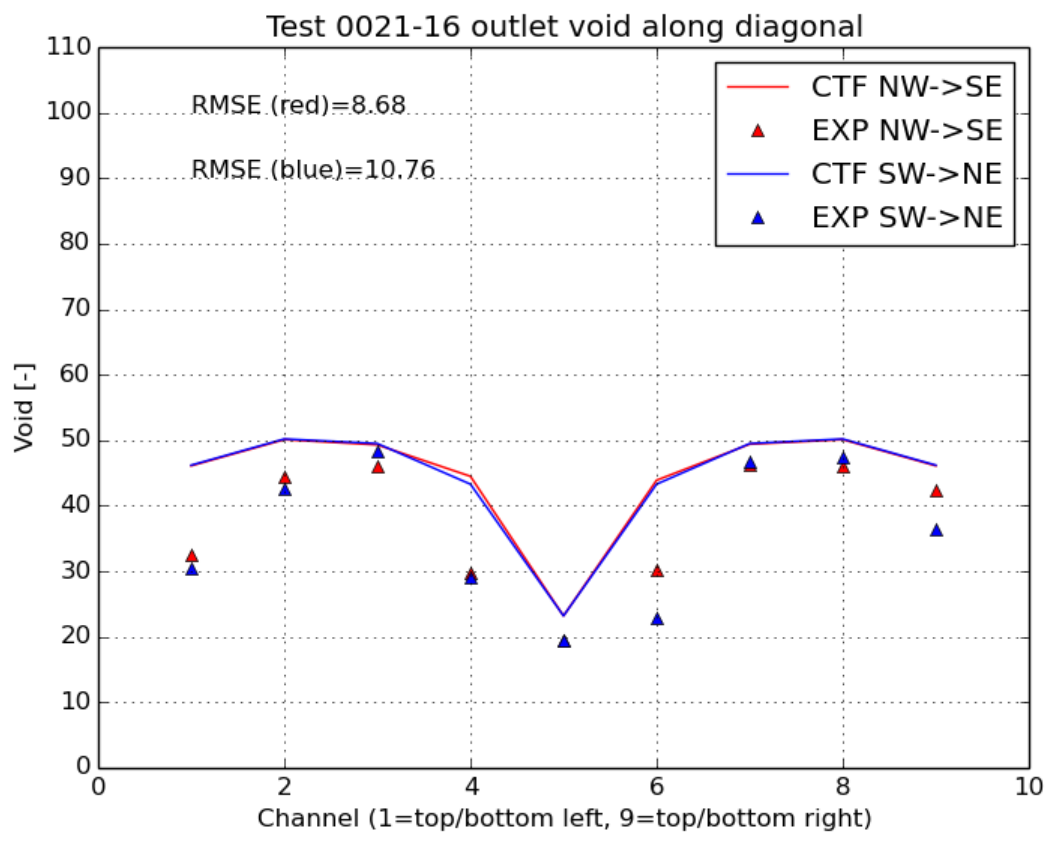

Figure 91. Measured and predicted void along diagonals of Test 0021-16.

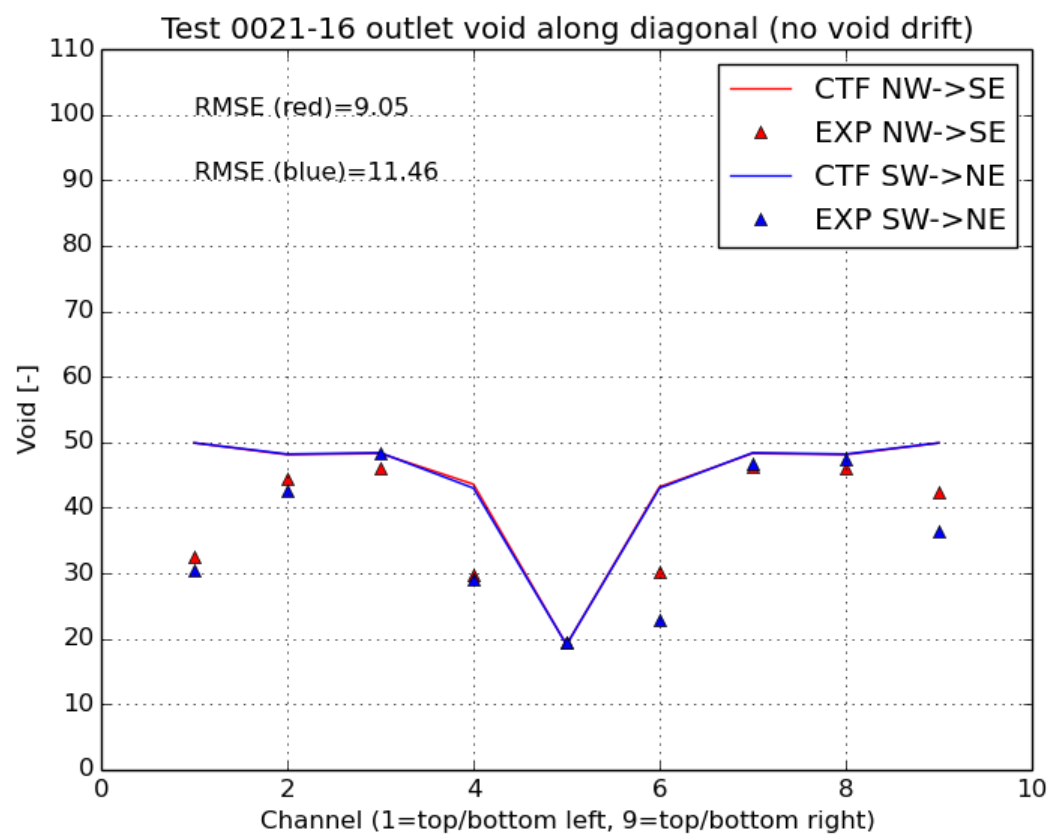

Figure 92. Measured and predicted void along diagonals of Test 0021-16 with void drift disabled. 


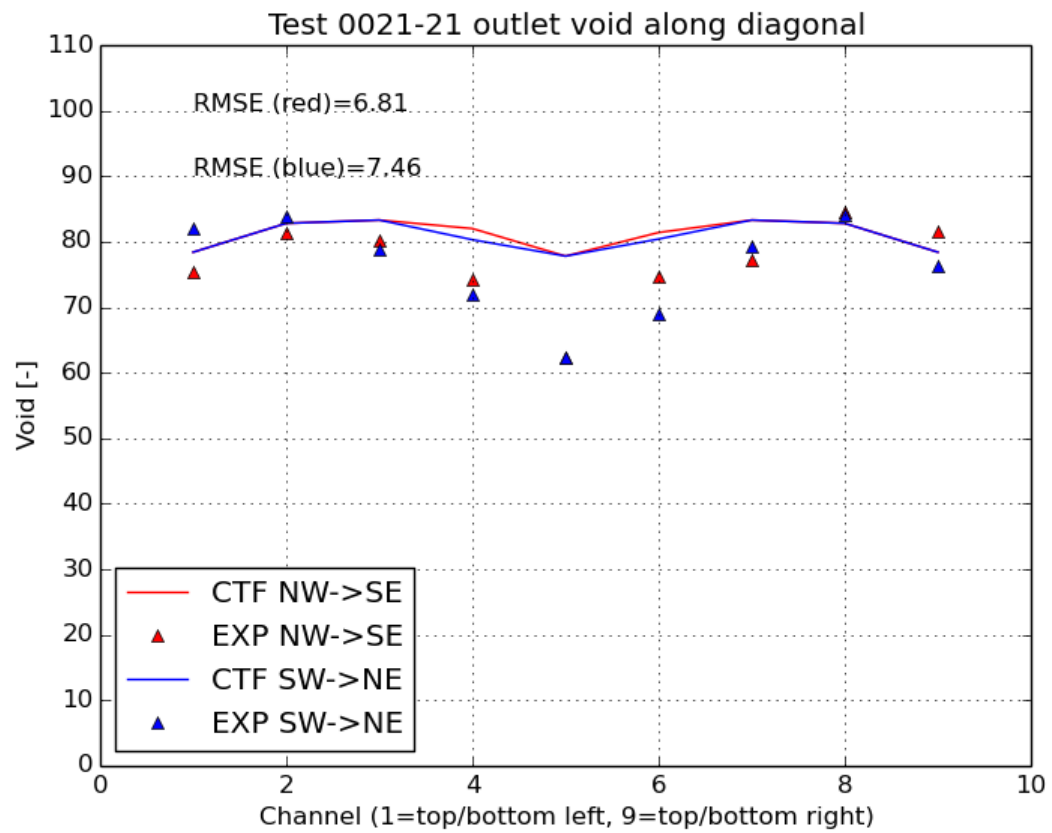

Figure 93. Measured and predicted void along diagonals of Test 0021-21.

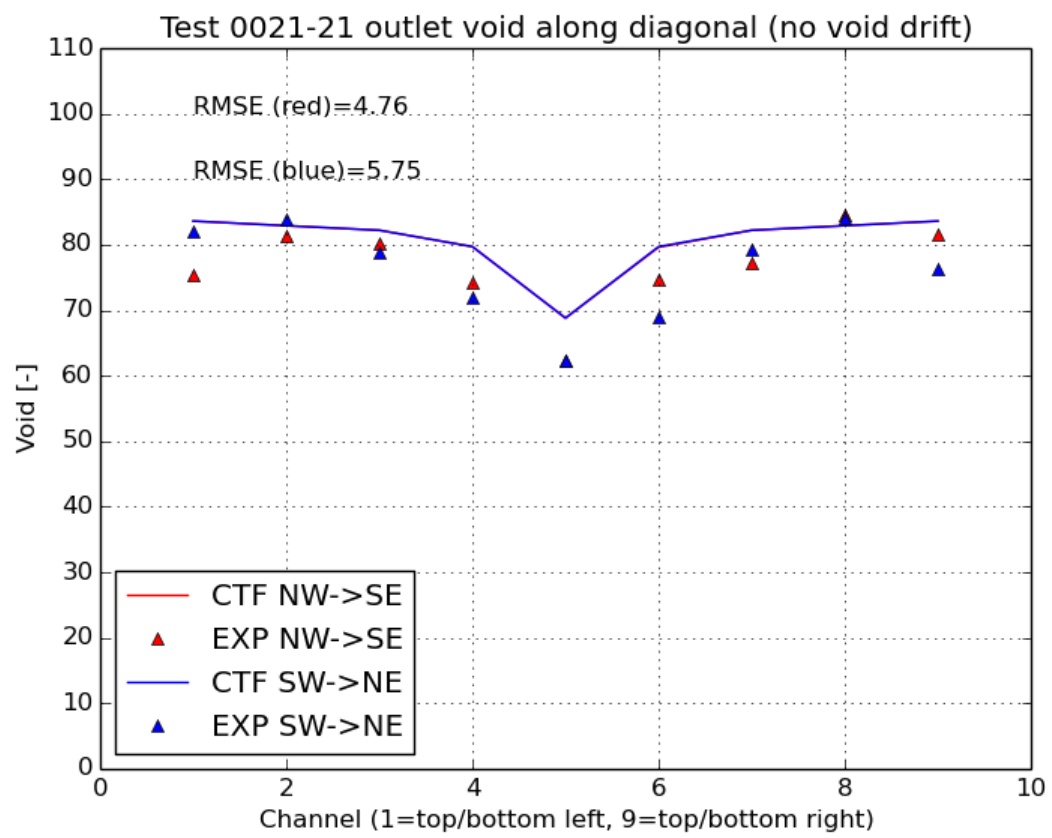

Figure 94. Measured and predicted void along diagonals of Test 0021-21 with void drift disabled. 


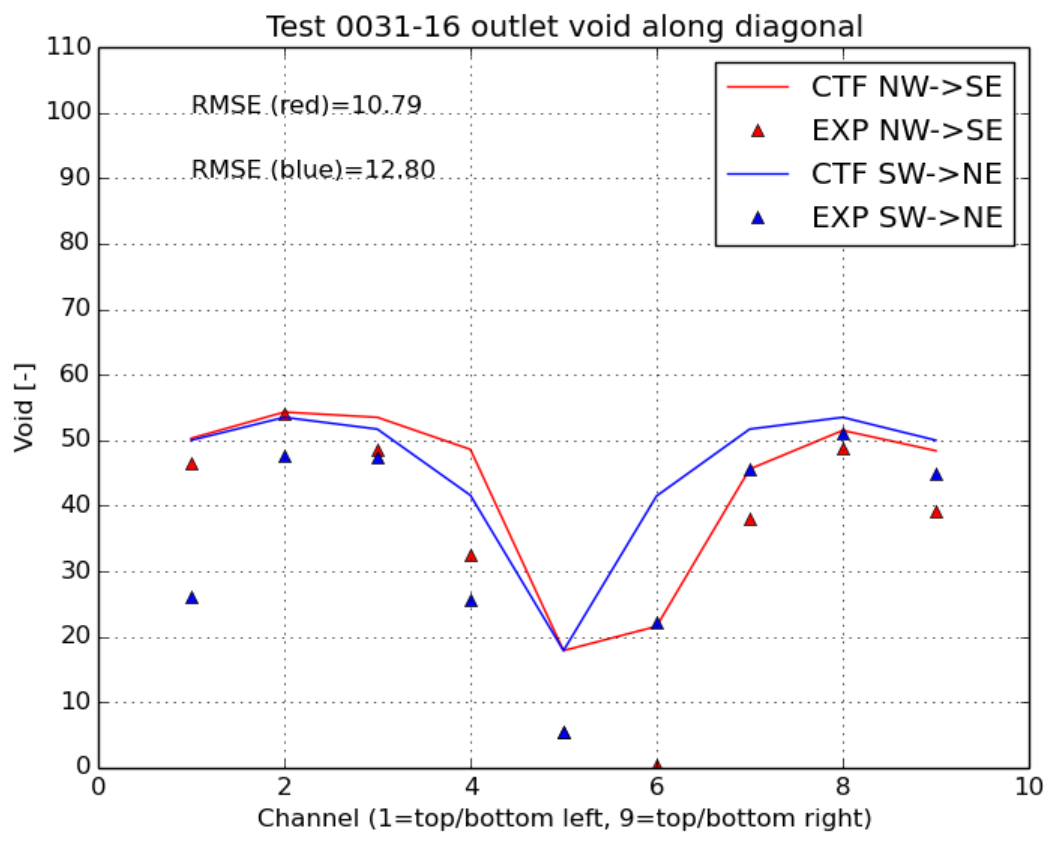

Figure 95. Measured and predicted void along diagonals of Test 0031-16.

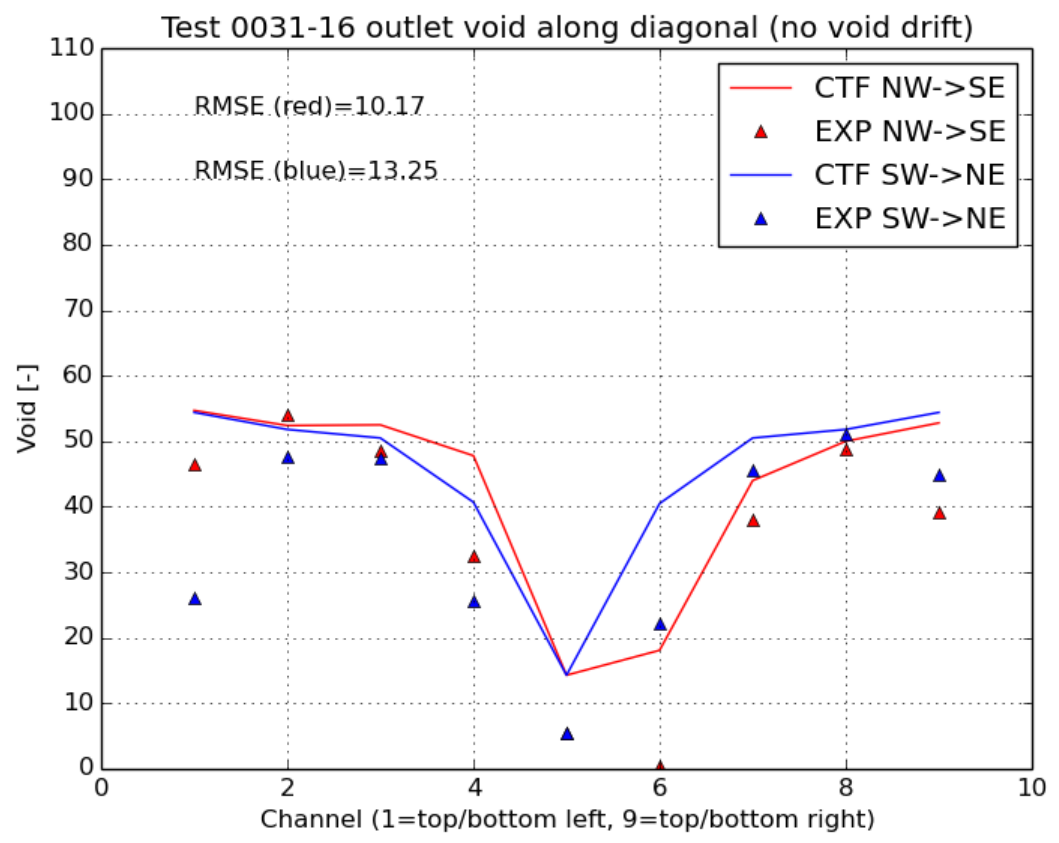

Figure 96. Measured and predicted void along diagonals of Test 0031-16 with void drift disabled. 


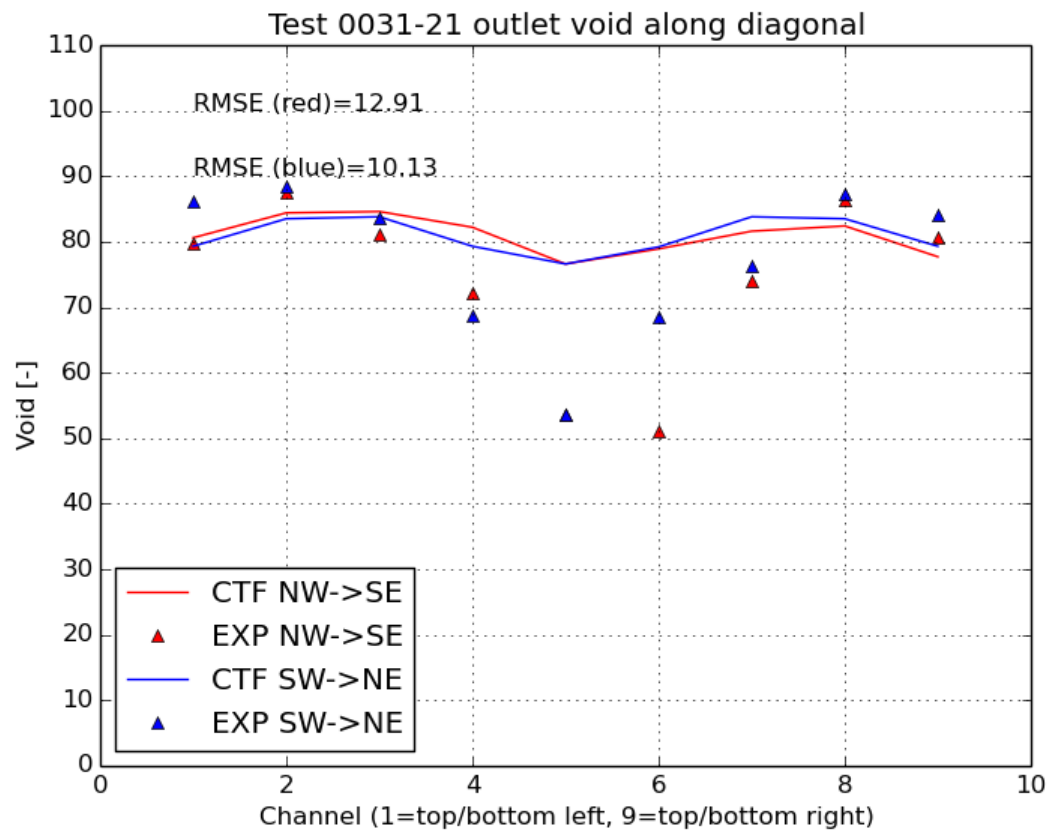

Figure 97. Measured and predicted void along diagonals of Test 0031-21.

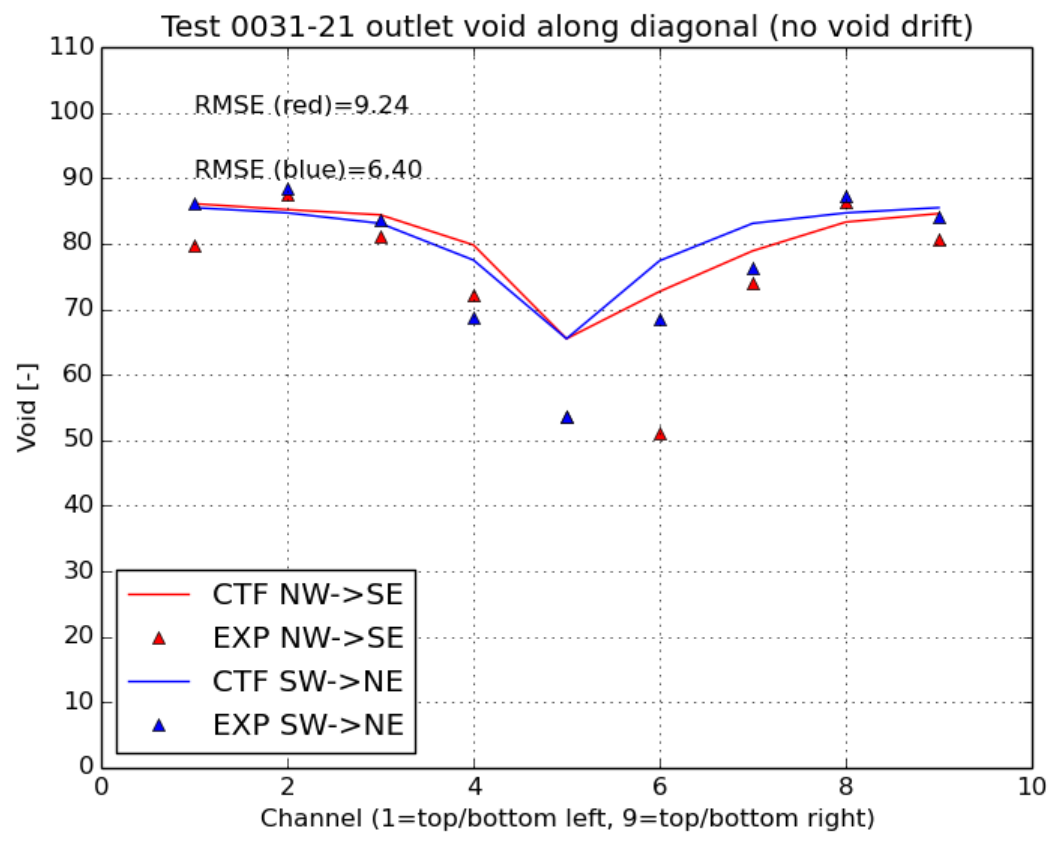

Figure 98. Measured and predicted void along diagonals of Test 0031-21 with void drift disabled. 


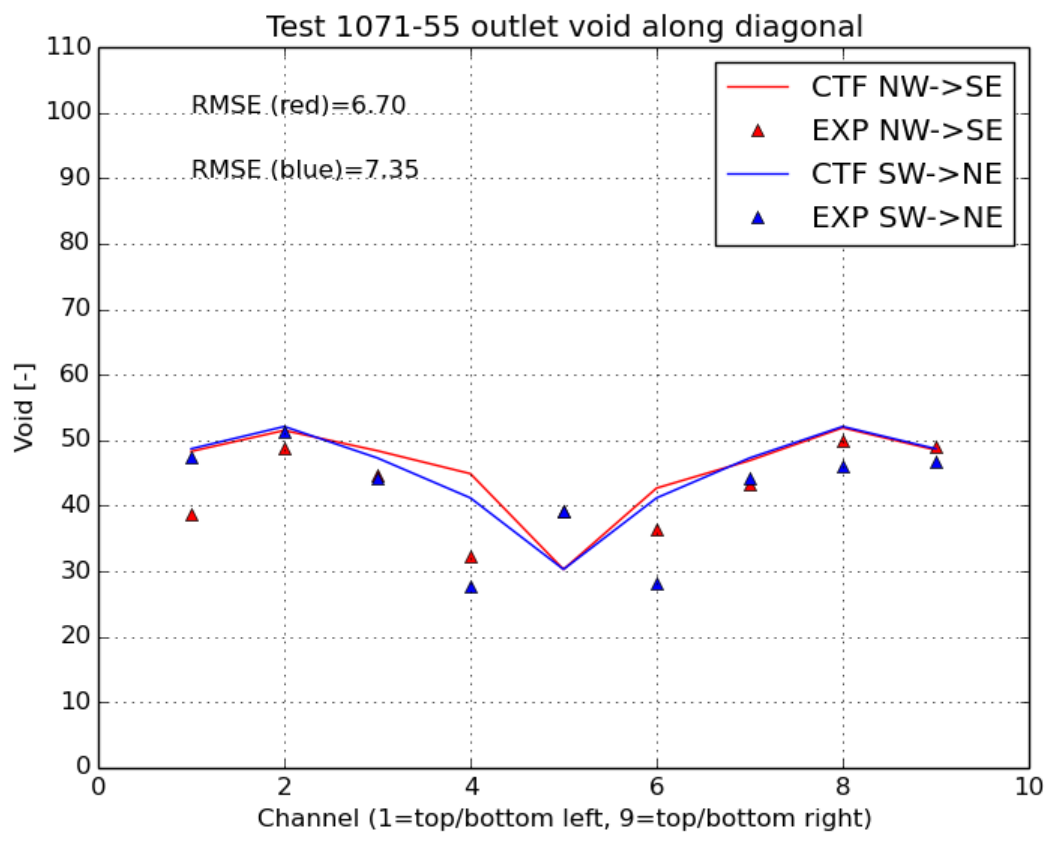

Figure 99. Measured and predicted void along diagonals of Test 1071-55.

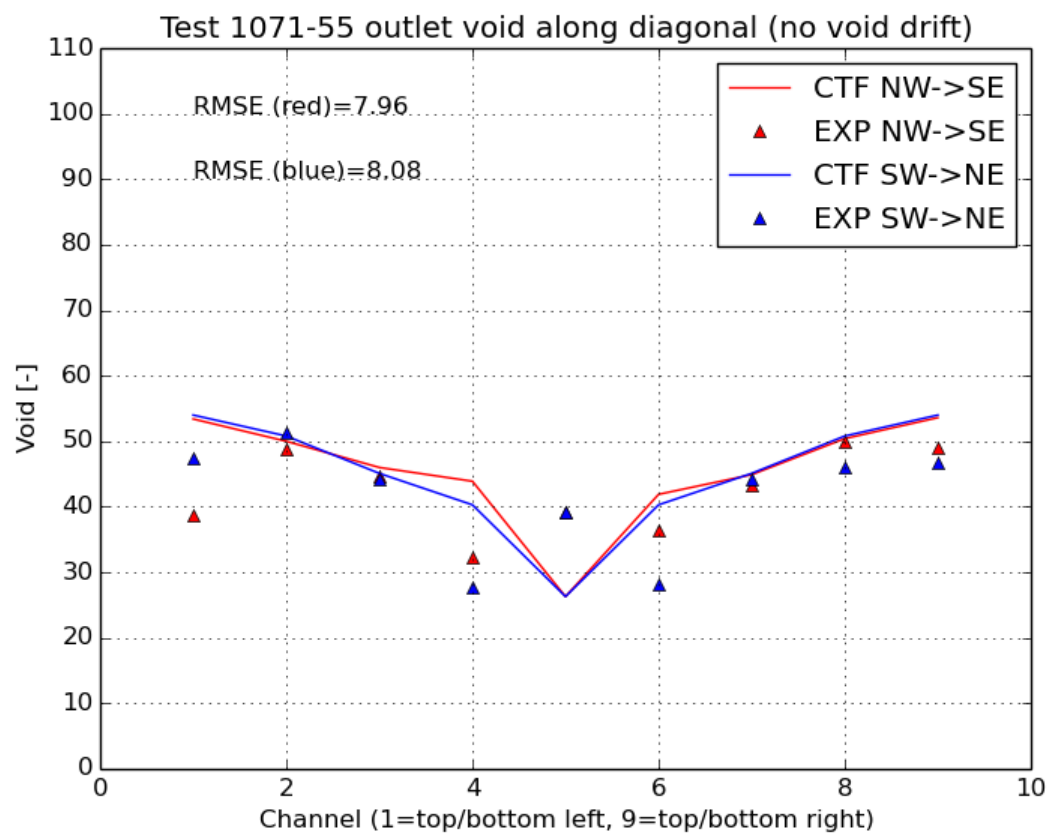

Figure 100. Measured and predicted void along diagonals of Test 1071-55 with void drift disabled. 


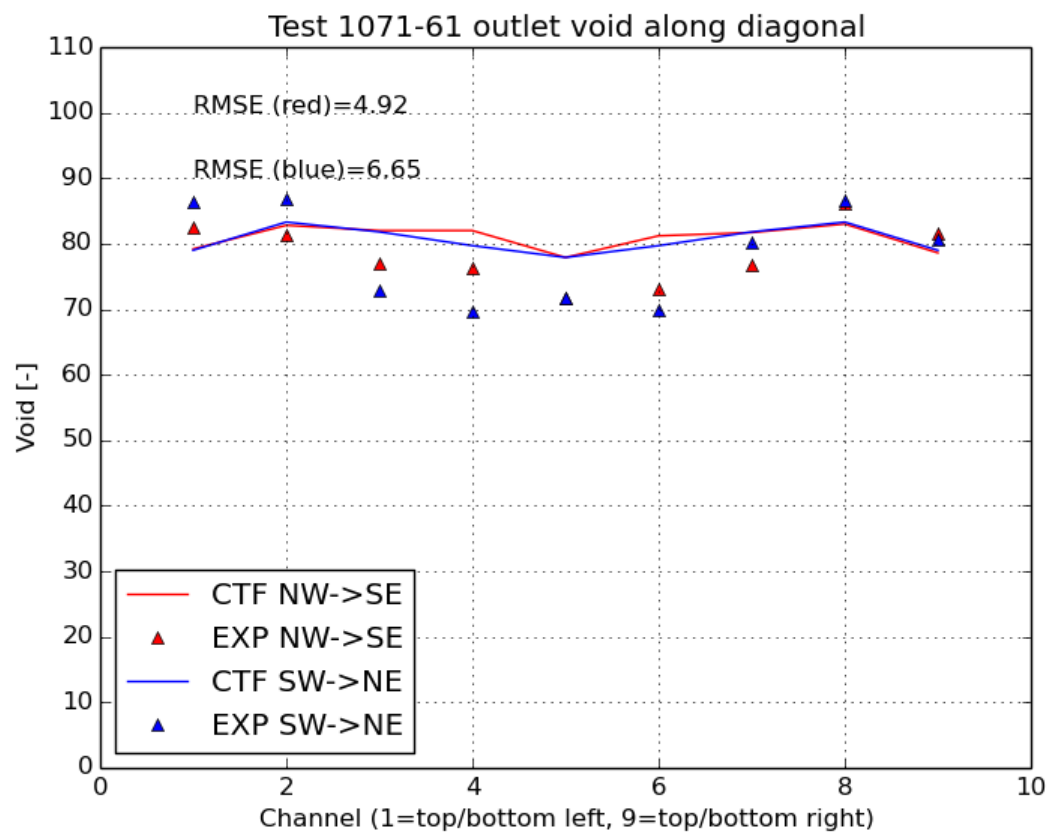

Figure 101. Measured and predicted void along diagonals of Test 1071-61.

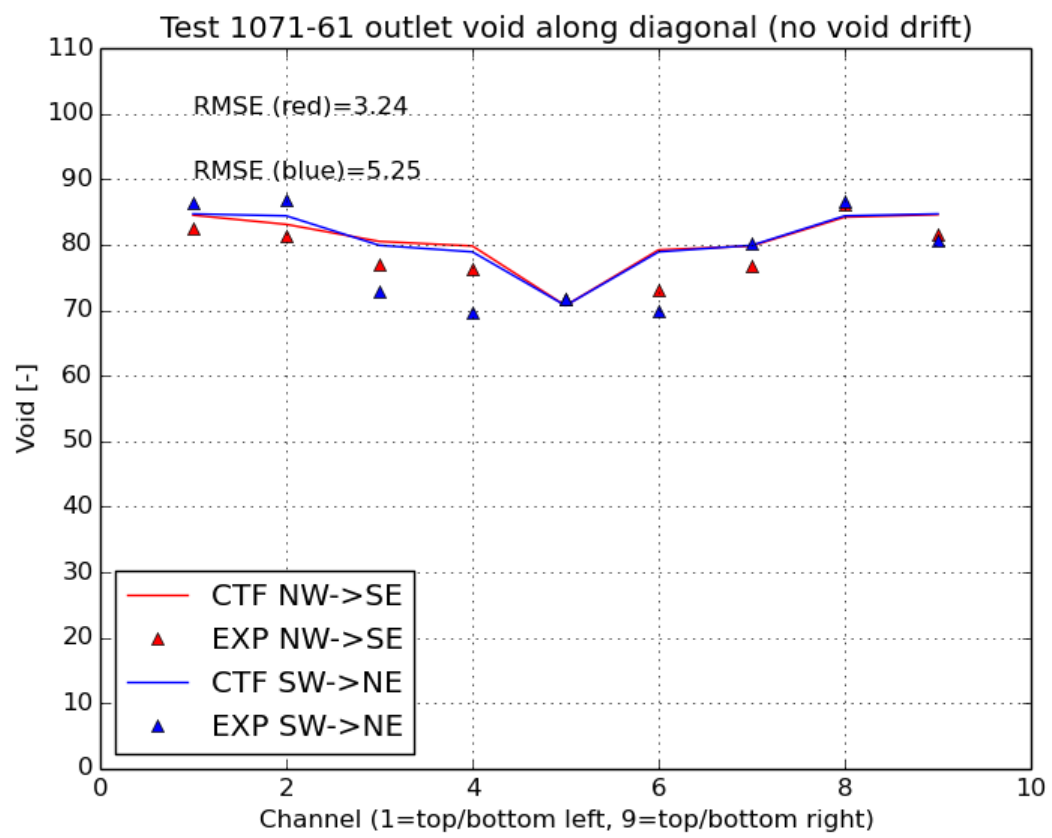

Figure 102. Measured and predicted void along diagonals of Test 1071-61 with void drift disabled. 


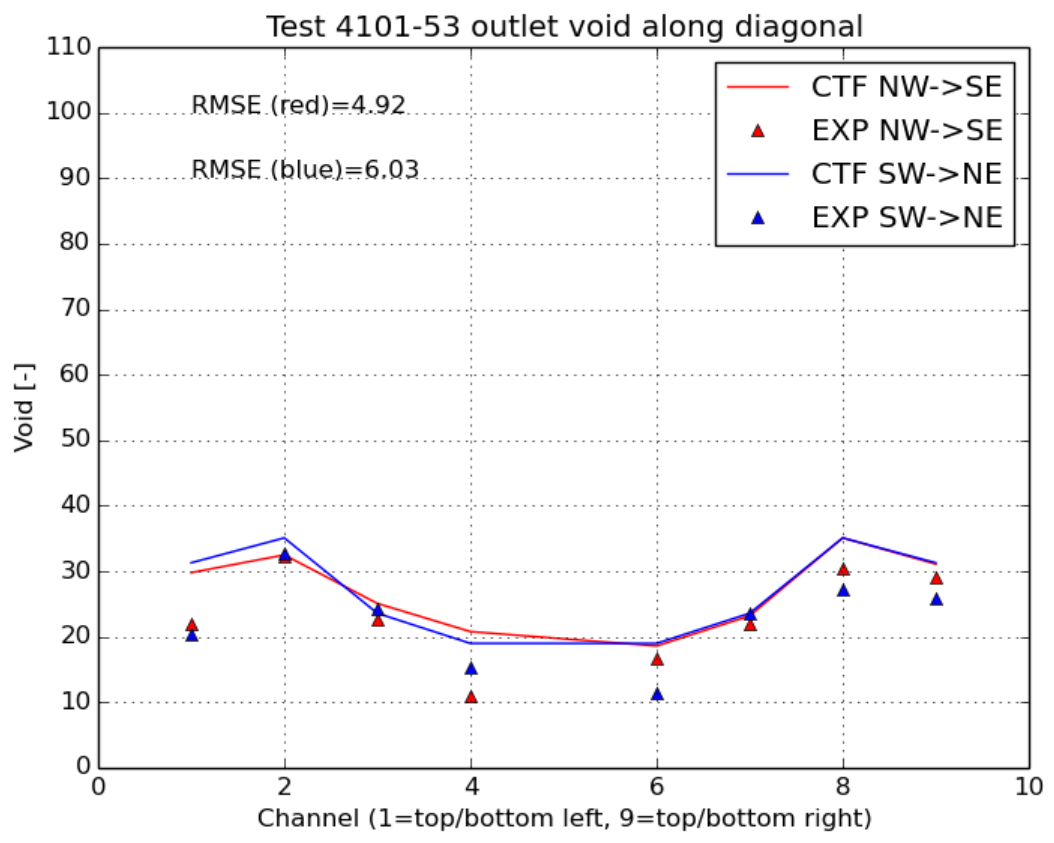

Figure 103. Measured and predicted void along diagonals of Test 4101-53.

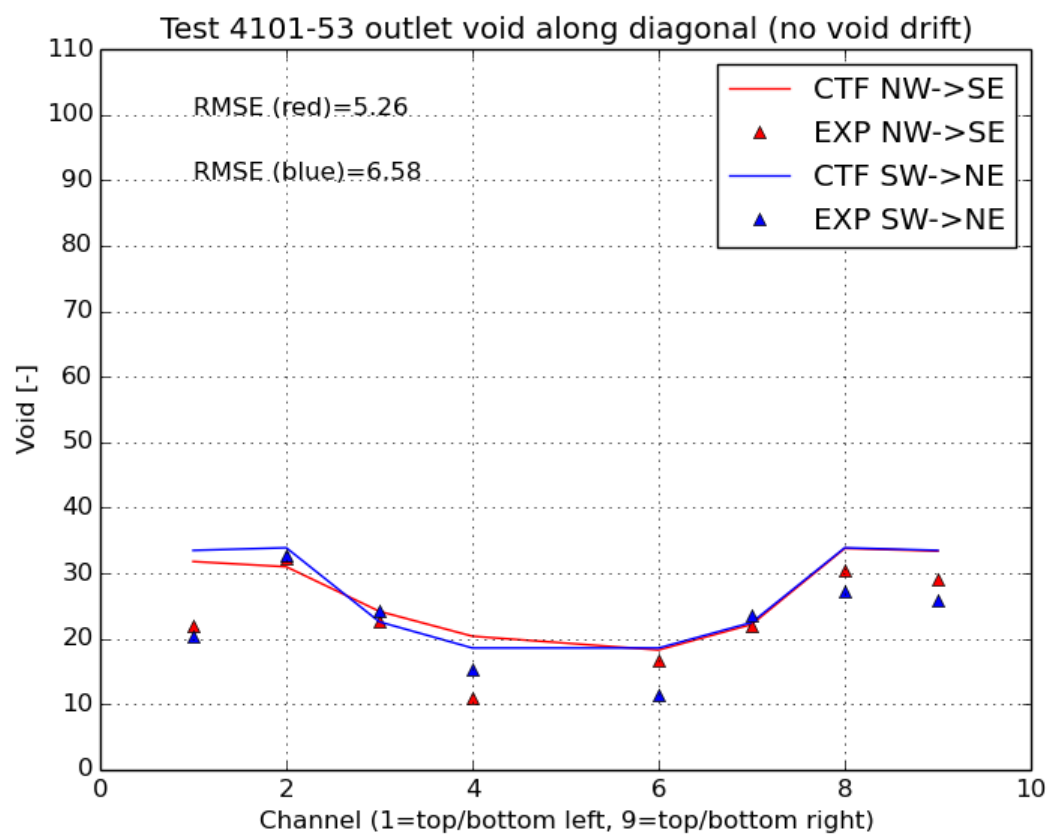

Figure 104. Measured and predicted void along diagonals of Test 4101-53 with void drift disabled. 


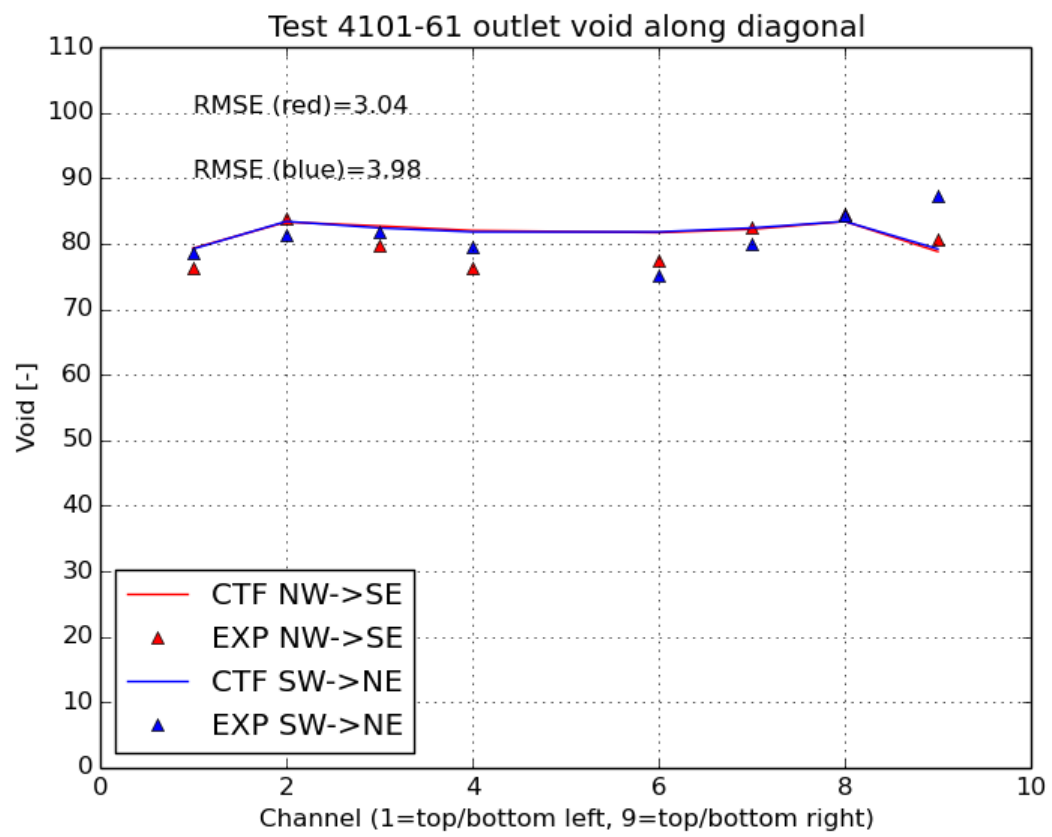

Figure 105. Measured and predicted void along diagonals of Test 4101-61.

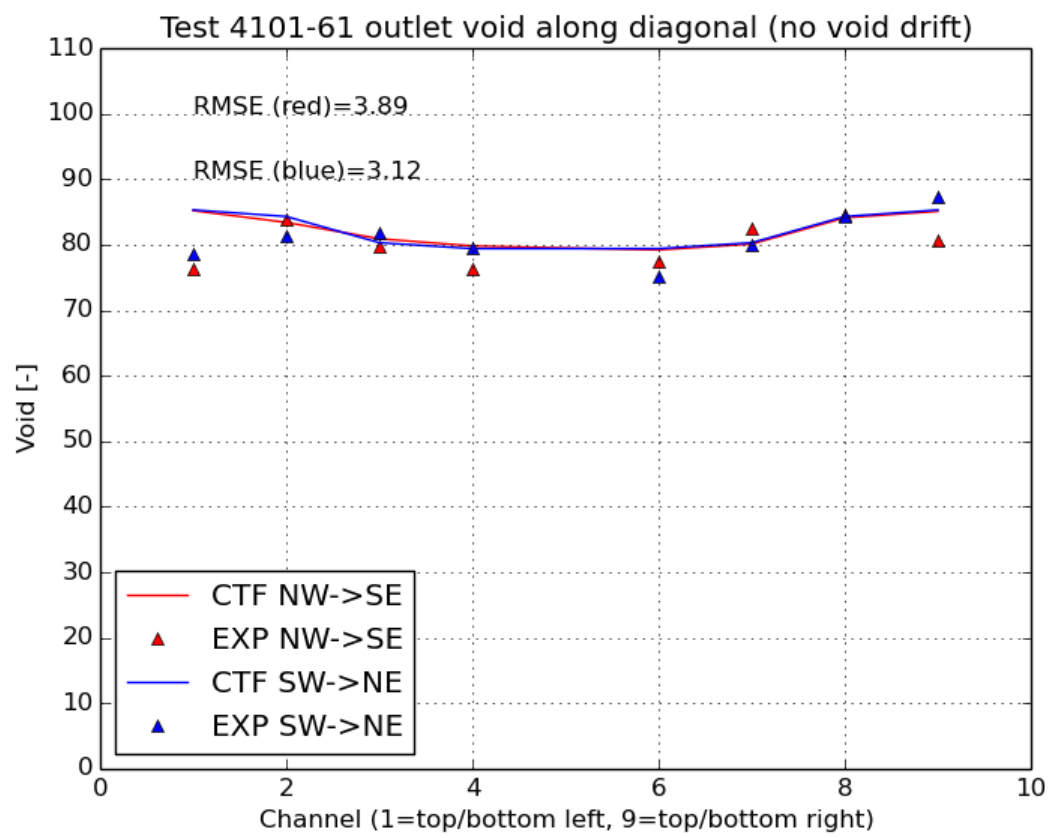

Figure 106. Measured and predicted void along diagonals of Test 4101-61 with void drift disabled. 


\section{CONCLUSION}

This report documents a study of the CTF turbulent mixing and void drift model. This study includes performing verification testing on the single-phase friction and single-phase turbulent mixing models, which affect the prediction of rod-bundle flow distribution. It is shown that both of these models are working as intended and compare favorably to analytical solutions.

Validation testing is performed to show how CTF-predicted flow distribution compares to experimental data for two facilities: the RPI $2 \times 2$ facility and the GE $3 \times 3$ facility. The flow reaches the equilibrium distribution for BWR geometry and operating conditions by the end of the $2 \mathrm{~m}$ length of the GE $3 \times 3$ test facility. In the low-pressure, air/water $2 \times 2$ facility, the flow distribution does not achieve equilibrium conditions until about $5 \mathrm{~m}$ of axial length. This result compares poorly with the experimental measurements, which were taken at the outlet of the $1 \mathrm{~m}$ facility.

Moving from single-phase $\mathrm{V} \& \mathrm{~V}$ testing to two-phase $\mathrm{V} \& \mathrm{~V}$ testing is challenging. First, twophase flow introduces the competing physical phenomenon of two-phase turbulent mixing and void drift, which cannot be separated in experiment. Therefore, individual models in CTF for modeling these effects cannot be separately tested. Second, it is found difficult to model low-pressure air/ water facilities in CTF; the code has a great deal of difficulty in meeting the specified steady-state convergence criteria of the code. After many unsuccessful attempts to model the $2 \times 2$ air/water facility, it is decided that CTF is not currently suitable for modeling the geometry and operating conditions of the facility. A study has been previously performed with this experiment by using an artificially elevated operating pressure of 100 bar; however, it is also noted that CTF does not seem to converge on a flow distribution that achieves equilibrium by the exit of the facility. Studies should be performed in the future to investigate the severity of the actual solution oscillations (rather than the engineering parameters used as convergence metrics) as well as the specific reasons why the CTF solution fails to converge.

Alternatively, success is found in modeling higher-pressure test facilities that are more representative of realistic reactor operating conditions. The Validation Manual includes results of the previously modeled GE $3 \times 3$ facility. This study expands on the previous study with new results and analysis. This study also adds the BFBT $8 \times 8$ void distribution experiments to the test matrix. The problem with these facilities is that they drive testing further from separate effects testing by adding additional complexities, including heating, boiling, implicit heat transfer, non-uniform enthalpy and density distribution leading to cross-flow, and spacer grid form losses in the flow. The benefit of the air/water tests is they have none of these complexities, allowing for a more direct assessment of the void drift and two-phase mixing model in CTF; however, as previously mentioned, CTF is not currently suitable for modeling air/water.

The BFBT tests add value to the test matrix because they include several features that are not covered by the GE tests, including larger test assembly, different spacer grid design, non-uniform axial and radial power distributions, different measurement technique, and a wider range of operating conditions. The BFBT study shows that the void drift model is effective in reducing the RMSE by several percentage points for lower void test cases ( 10-50\% void). At higher void cases $(\sim 80 \%)$, the radial void distribution tends to be more uniform, and it is consistently seen that the void drift model has a relatively insignificant effect on the accuracy of the results.

In several high void cases, accuracy actually improves with the void drift model disabled. However, it is important to note CTF's general tendency to overpredict void in the unheated sections of the bundle, which has a large impact on this observed trend. Disabling the void drift model keeps void from migrating into the center of the bundle and allows CTF predicted void to drop lower in the unheated center. This region is where most prediction improvements are observed and only for the higher void cases. Similar to the conclusion of the GE validation testing, it is found that greatest improvements to predictive capabilities are made for the boundary channels when the void drift model is enabled.

It is found that the optimum single-phase mixing coefficient is $\beta=0.007$ according to comparisons with the $2 \times 3$ mixing tests. A previous CASL study [10] suggested an optimum value of 0.0044 . Since the mixing coefficient is dependent on actual gap geometry and flow conditions, this range of values provides bounds on values to use for light water reactor (LWR) analysis. It is found that the 
predictive Rogers \& Rosehart model tends to over-predict single phase mixing. This degrades the accuracy of the results for the GE $3 \times 3$ single-phase cases compared with using the constant value of 0.007. It is found that switching between the two different single-phase mixing models have almost no impact on results accuracy for the two-phase GE $3 \times 3$ cases.

The completion of this study results in a more rigorous testing of the void drift model in CTF as well as supporting models that affect the prediction of void drift, including friction and turbulent mixing. The CTF Verification Manual [16] has been updated with the verification tests discussed in this document. The CTF Validation Manual has been updated with the BFBT study discussed in this document. Selected BFBT validation cases have been added to automated CTF regression testing to ensure that the results of this study remain valid.

\section{References}

[1] R. Lahey and F. Moody. The Thermal-Hydraulics of a Boiling Water Nuclear Reactor. Tech. rep. ANS Monograph, 1977.

[2] R.K. Salko and M.N. Avramova. CTF Theory Manual. The Pennsylvania State University. 2012.

[3] R. Sterner and R. Lahey. Air/Water Subchannel Measurements of the Equilibrium Quality and Mass Flux Distribution in a Rod Bundle. Tech. rep. Rensselaer Polytechnic Institute, 1983.

[4] R. Salko et al. CTF Validation Manual. Tech. rep. CASL-U-2014-0169-000. Consortium for Advanced Simulation of Light Water Reactors, 2015.

[5] N. Todreas and M. Kazimi. Nuclear Systems II: Elements of Thermal Hydraulic Design. Hemisphere Publishing Corporation, 1990.

[6] M. Sadatomi et al. "Single- and Two-Phase Turbulent Mixing Rate between Adjacent Subchanels in a Vertical $2 \times 3$ Rod Array Channel". In International Journal of Multiphase Flow 30 (2004), pp. 481-498.

[7] M. Sadatomi et al. "Flow Characteristics in Hydraulically Equilibrium Two-Phase Flows in a Vertical $2 \times 3$ Rod Bundle Channel”. In International Journal of Multiphase Flow 30 (2004), pp. 1093-1119.

[8] M. Sadatomi, Y. Sato, and S. Saruwatari. "Two-Phase Flow in Vertical Noncircular Channels". In International Journal of Multiphase Flow 8 (1982), pp. 641-645.

[9] Z. Karoutas et al. Subcooled Boiling Data from Rod Bundles. Tech. rep. 1003383. EPRI, 2002.

[10] Y. Sung et al. Application of Multi-Scale Thermal-Hydraulic Models to DNB Analysis. Tech. rep. CASL-U-2014-0119-000. Consortium for Advanced Simulation of Light Water Reactors, 2014.

[11] R. Lahey Jr., B. Shirlakar, and D. Radcliffe. Two-Phase Flow and Heat Transfer In Multirod Geometries: Subchannel and Pressure Drop Measurements in a Nine-Rod Bundle for Diabatic and Adiabatic Conditions. Tech. rep. General Electric, 1970.

[12] B. Neykov et al. NUPEC BWR Full-size Fine-mesh Bundle Test (BFBT) Benchmark. Tech. rep. Nuclear Energy Agency, 2006.

[13] B. Shiralkar and D. Radcliffe. An Experimental and Analytical Study of the Synthesis of Grid Spacer Loss Coefficients. Tech. rep. NEDE-13181. General Electric, 1971.

[14] M. Avramova, K. Ivanov, and L. Hochreiter. "Analysis of Steady State and Transient Void Distribution Predictions for Phase I of the OECD/NEA BFBT Benchmark using CTF/NEM". In The 12th International Topical Meeting on Nuclear Reactor Thermal Hydraulics (NURETH12). 2007.

[15] B. Neykov, M. Avramova, and K. Ivanov. "Phase I-Void Distribution Final Results". In $6^{\text {th }}$ OECD/NRC BFBT Workshop. 2009. 
[16] R. Salko, C. Jernigan, and J. Kelly. CTF Verification Manual. Tech. rep. CASL-U-2015-XXXX000. Consortium for Advanced Simulation of Light Water Reactors, 2015. 